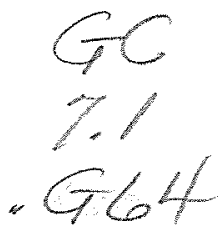

\title{
Steady Models of Arctic Shelf-Basin Exchange
}

by

\author{
Daniel Reed Goldner
}
B.A. Earth and Planetary Science
Harvard University, 1991

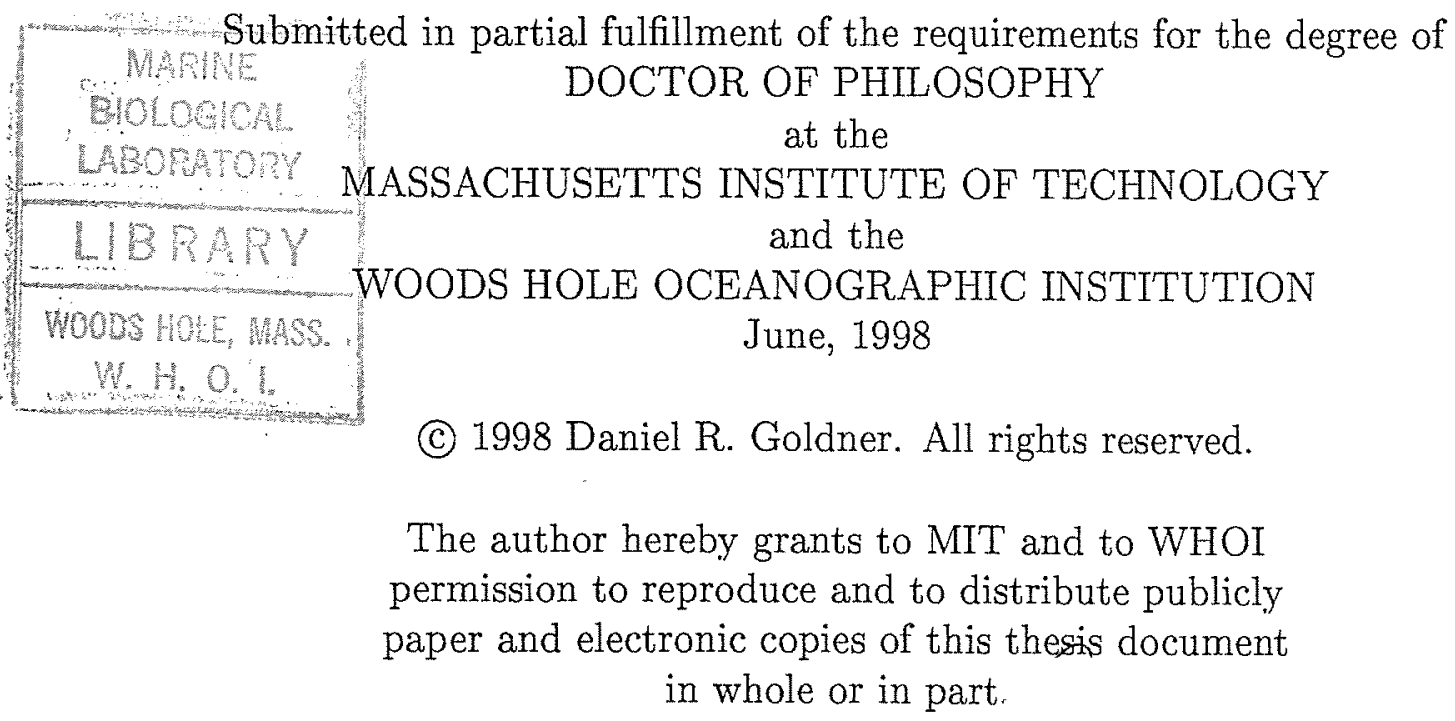

Signature of Author:

Joint Program in Physical Oceanography Massachusetts Institute of Technology Woods Hole Oceanographic Institution

Anril 30. 1998

Certified by:

David C. Chapman

Senior Scientist

Thesis Superviser

Accepted by:

W. Brechner Owens

Chairman, Joint Committee for Physical Oceanography Massachusetts Institute of Technology Woods Hole Oceanographic Institution 


\title{
Steady Models of Arctic Shelf-Basin Exchange
}

\author{
by \\ Daniel Reed Goldner
}

\begin{abstract}
Submitted to the Joint Committee for Physical Oceanography of the Massachusetts Institute of Technology and the Woods Hole Oceanographic Institution on April 30, 1998 in Partial Fulfillment of the Requirements for the Degree of Doctor of Philosophy
\end{abstract}

\begin{abstract}
Efforts to understand the Arctic system have recently focused on the role in local and global circulation of waters from the Arctic shelf seas. In this study, steadystate exchanges between the Arctic shelves and the central basins are estimated using an inverse box model. The model accounts for data uncertainty in the estimates, and quantifies the solution uncertainty. Other features include resolution of the two-basin Arctic hydrographic structure, two-way shelf-basin exchange in the surface mixed layer, the capacity for shelfbreak upwelling, and recognition that most inflows enter the Arctic via the shelves. Aggregate estimates of all fluxes across the Arctic boundary, with their uncertainties, are generated from flux estimates published between 1975 and 1997. From the aggregate estimates, mass-, heat-, and salt-conserving boundary flux estimates are derived, which imply a net flux of water from the shelves to the basins of $1.2 \pm 0.4 \mathrm{~Sv}$. Due primarily to boundary flux data uncertainty, constraints of mass, heat, and salt conservation alone cannot determine how much shelf-basin exchange occurs via dense overflows, and how much via the surface mixed layer. Adding $\delta^{18} \mathrm{O}$ constraints, however, greatly reduces the uncertainty. Dense water flux from the shelves to the basins is necessary for maintaining steady state, but shelfbreak upwelling is not required. Proper representation of external sources feeding the shelves, rather than the basins, is important to obtain the full range of plausible steady solutions. Implications of the results for the study of Arctic change are discussed.
\end{abstract}

Thesis Supervisor:

David C. Chapman

Senior Scientist

Woods Hole Oceanographic Institution 


\section{Acknowledgments}

The single most important factor contributing to the wonderful experience I've had in the Joint Program has without doubt been my advisor, Dave Chapman. I am grateful for his encouragement when I was stuck, for his superior example in project planning and in writing, for his flexibility in letting me work from Boston and then from Venice, for his well-known dedication to general student welfare, and most of all, for his ardent support of the often neglected idea that family is more important than work. I've lost track of the number of times when, in talking with a stymied classmate, I've been most helpful by quoting Dave.

Many others have contributed to my Joint Program experience, and to this work. I particularly appreciate the time, interest, and advice given to me by Carl Wunsch, Jochem Marotzke, Glen Gawarkiewicz. and Breck Owens, and I am especially grateful to Paola Rizzoli for her ongoing encouragement and assistance. Thanks also to K Aagaard, D Battisti, D Bauch, J Blindheim, A Foldvik, G Maykut, S Pfirman, B Rudels, P Schlosser, A Solow, D Thomas, and T Vinje for taking time to provide helpful information and clarification. I would also like to thank Joe Pedlosky, Steve Lentz, John Marshall, and Jim Price for providing excellent (but diverse!) models of great teaching. The unfailing assistance of Ronni Schwartz and the WHOI Education Office is gratefully acknowledged.

Many thanks to A Bergamasco, G Dallaporta, J Zanin, S Carniel, F DeBiasio, and G Ferro Milone at the Instituto per lo Studio della Dinamica delle Grandi Masse, Venice, Italy, for their hospitality, and to Marco Marani and the Marani family for their support and friendship. My friends and colleagues in the Joint Program and in EAPS are to be thanked, both for warm comraderie, and for providing through inspired discussion most of my education. Melissa Bowen and Richard Wardle are especially acknowledged, with thanks for five years of rewarding collaboration and friendship. Deepest gratitude and much love go to my family, Michael and Barbara Goldner, Emily Goldner, and Peter Jaffe. Above all, unending love and thanks to Dr. Michele Jaffe.

This work was supported by National Science Foundation grant OPP-9422292 as part of the Arctic System Science (ARCSS) program, administered by the Office of Polar Programs. 


\section{Contents}

$\begin{array}{ll}\text { Acknowledgments } & 3\end{array}$

1 Introduction $\quad 7$

1.1 The Arctic Ocean . . . . . . . . . . . . . . . 7

1.2 Arctic Shelf-Basin Exchange . . . . . . . . . . . . . . 12

1.3 Previous Work . . . . . . . . . . . . . . . . . 14

1.4 Present Work . . . . . . . . . . . . . . . 17

2 Box Model Description 19

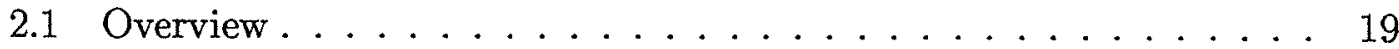

2.2 Structure ...................... 23

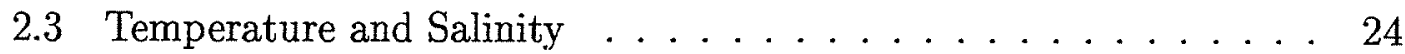

2.4 Oxygen Isotope Ratio . . . . . . . . . . . . . . . . . 26

2.5 Uncertainty ........................ 29

2.6 Forcing . . . . . . . . . . . . . . . . 29

2.6.1 Forcing affecting one box . . . . . . . . . . . 30

2.6.2 Forcing affecting more than one box: outflows . . . . . . 30

2.6.3 Forcing affecting more than one box: inflows . . . . . . . . 32

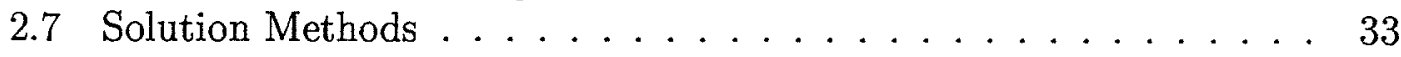

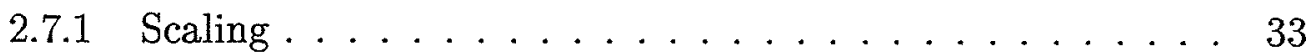

2.7.2 Rank determination . . . . . . . . . . . . . . 33

2.7.3 Optimal solutions: non-negative least squares and Monte Carlo 35

2.7.4 Extreme solutions: linear programming . . . . . . . 37

3 Boundary Fluxes 39

3.1 Introduction . . . . . . . . . . . . . . . . 39

3.2 Notation and Terms . . . . . . . . . . . . . 42

3.3 Previous Estimates . . . . . . . . . . . . . . . . . . . . . . 44

3.3 .1 Oceanic fluxes . . . . . . . . . . . . . 45

3.3 .2 Ice fluxes ..................... 49

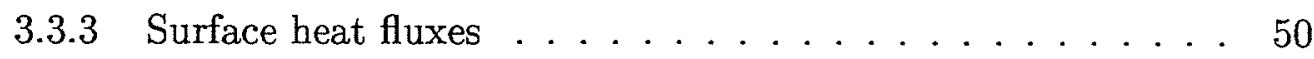

3.4 Aggregate Estimates . . . . . . . . . . . . . . . 53 
3.4 .1 Volume fluxes . . . . . . . . . . . . . . . 53

3.4.2 Potential temperature and salinity anomaly fluxes . . . . . 57

3.5 Budgets . . . . . . . . . . . . . . . 63

3.5.1 Budget 1: Aggregate estimates only . . . . . . . . . 63

3.5.2 Budget 2: Aggregate estimates with mass conservation . . 65

3.5.3 Budget 3: Aggregate estimates with mass, heat, and salt

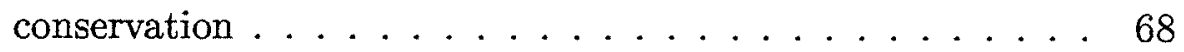

3.6 Discussion . . . . . . . . . . . . . . . . 70

3.7 Summary . . . . . . . . . . . . . . . 74

4 Estimates of Shelf-Basin Exchange $\quad 76$

4.1 Introduction . . . . . . . . . . . . . . 76

4.2 Estimates from Temperature and Salinity . . . . . . . . . 77

4.2 .1 Optimal solution . . . . . . . . . . . . 77

4.2 .2 Uncertainty of optimal solution . . . . . . . . . 79

4.2 .3 Extreme solutions . . . . . . . . . . . . . . . . 85

4.2.4 Sensitivity to prescribed temperatures and salinities . . . . 89

4.3 Estimates from Temperature, Salinity, and $\delta^{18} \mathrm{O} \ldots \ldots \ldots . . . .91$

4.3.1 Optimal solution . . . . . . . . . . . . . . . . 92

4.3.2 Uncertainty of optimal solution . . . . . . . . . . . 92

4.3.3 Extreme solutions . . . . . . . . . . . . . . . . . 96

4.3.4 Sensitivity to prescribed $\delta^{18} \mathrm{O} \ldots \ldots \ldots \ldots . \ldots 98$

4.4 Effects of the Model Assumptions . . . . . . . . . . . . . 100

4.4 .1 Representation of river runoff . . . . . . . . . . 100

4.4.2 Representation of the Arctic Archipelago . . . . . . . . . 101

4.4.3 Representation of the West Spitzbergen Current . . . . . . . 104

4.4 .4 Representation of diffusion . . . . . . . . . . . . 106

4.4.5 Inclusion of cross-shelfbreak upwelling . . . . . . . 107

4.4.6 Inclusion of ice melt and advection . . . . . . . . . . . 109

4.4.7 Sensitivity to prescribed basin surface heat flux . . . . . 110

4.4.8 Sensitivity to prescribed uncertainty . . . . . . . . 112

4.5 Steady Solutions for Alternative Arctics . . . . . . . . . . 114

4.5 .1 Runoff volume. . . . . . . . . . . . . . . 114

4.5.2 Net ice formation . . . . . . . . . . . . . . 115

4.5.3 Atlantic Layer temperature . . . . . . . . . . . . 116

4.6 Summary . . . . . . . . . . . . . . . . 117

5 The Role of the Arctic Shelves: Revisiting Past Results 118

5.1 Introduction: the Distillery Shelf Model . . . . . . . . . . . 118

5.2 Inversions with the Distillery Shelf . . . . . . . . . . 119

5.2 .1 One basin . . . . . . . . . . . . . 120 
5.2 .2 Two basins . . . . . . . . . . . . . . . . . . 124

5.2 .3 Summary: distillery shelf inversions . . . . . . . . . . 126

5.3 A Toy Model . . . . . . . . . . . . . . . . . . . 127

5.4 Discussion . . . . . . . . . . . . . . . . . 132

5.5 Summary . . . . . . . . . . . . . . . . . 135

6 Summary and Discussion 137

6.1 Summary of Results . . . . . . . . . . . . . . . 137

6.2 The Steady Arctic . . . . . . . . . . . . . . . 140

6.3 Steady Models and Arctic Change . . . . . . . . . . . . 150

6.4 Closing . . . . . . . . . . . . . . . . . . . . 152

A Solution Uncertainty in Non-Negative Least Squares 153

B Constraining Ice Formation with the Estimates of Thomas et al. 157

$\begin{array}{ll}\text { References } & 159\end{array}$ 


\section{Chapter 1}

\section{Introduction}

The wide shelf seas of the Arctic Ocean are fed by the Pacific and Atlantic Oceans, as well as $10 \%$ of the world's river runoff. On the shelves, mixing, contact with shelf sediments, and seasonal freezing and melting all transform the character of the water, which then flows from the shelves to the Arctic basins and out to the world ocean. A transformative threshold at the Arctic boundary, the shelves and their interactions with the basins have recently become a focus of efforts to understand the role of the Arctic in the world ocean. The goal of this thesis is to estimate how much shelf-basin exchange is required to maintain the Arctic Ocean in steady state, and to show how data uncertainty and assumptions about the nature of shelf-basin exchange influence that estimate.

\subsection{The Arctic Ocean}

The Arctic is a tiny ocean, with only $4 \%$ of the world ocean surface area and $1 \%$ of the world ocean volume. The Lomonosov Ridge, with a sill depth of approximately $1400 \mathrm{~m}$, divides the Arctic into the 3500-meter deep Canadian Basin, and the 4500-

meter deep Eurasian Basin (Figure 1.1). The basins are nearly surrounded by the 


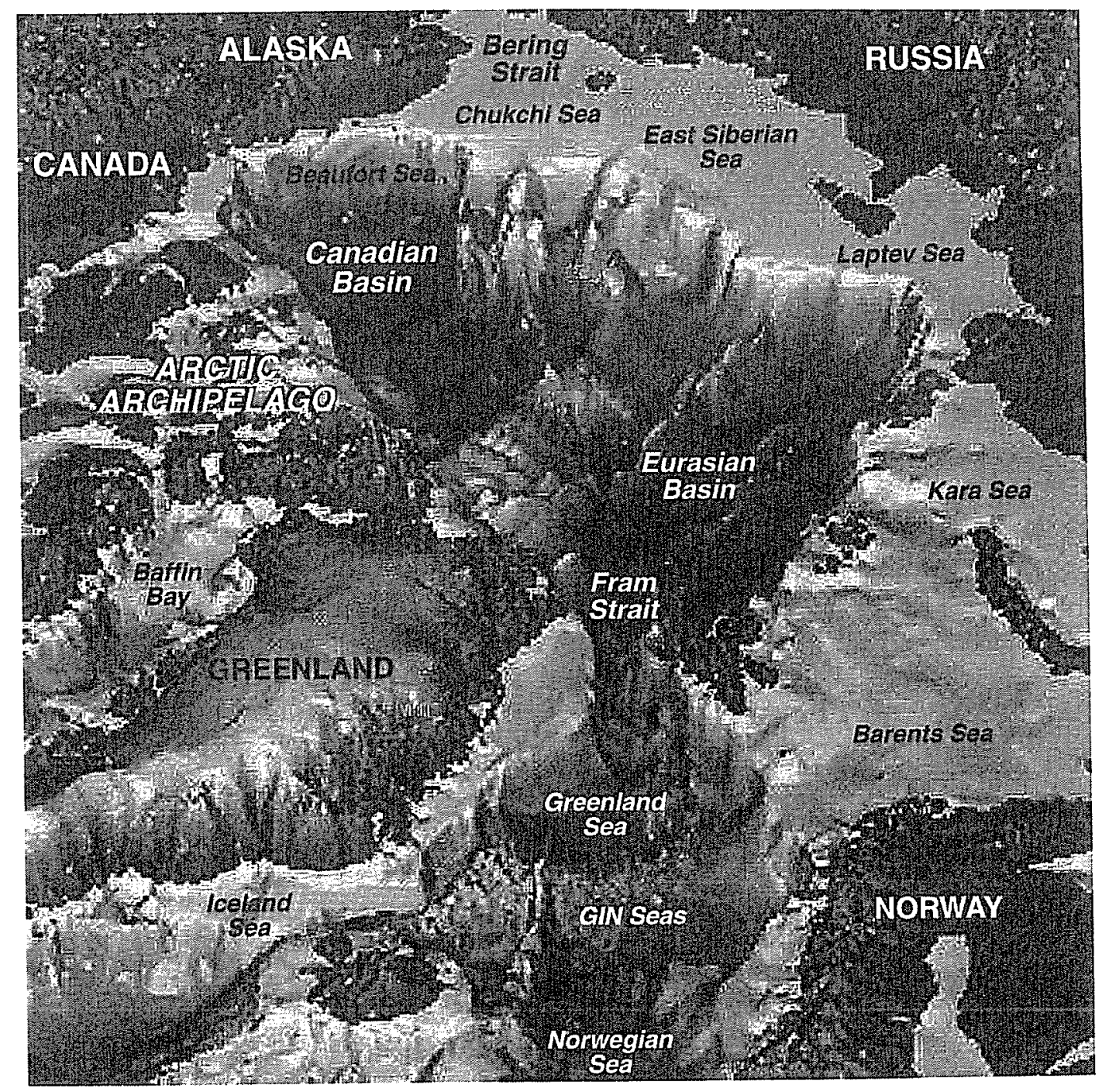

Figure 1.1: The Arctic Ocean (after Gore-Chernomyrdin Commission Environmental Working Group, 1997). 
shelf seas, up to $800 \mathrm{~km}$ wide, which occupy one third of the Arctic Ocean and represent $25 \%$ of the world's shelf area.

The Arctic basins are permanently ice-covered, except for narrow, transitory leads which expose approximately $1 \%$ of the basin surface area at any given time. In general, ice in the basin flows from the Canadian Basin over the North Pole to the Eurasian Basin and out the Fram Strait, carried by a surface current known as the Transpolar Drift. The shelves are generally open in summer and refreeze each fall. Ice formation continues all winter long, and is especially intense in coastal polynyas, open water areas created when the wind blows new ice away from shore. See Smith et al. (1990) and Maykut (1985) for more on leads, polynyas, and sea ice.

Approximately one third of the flow into or out of the Arctic passes over the shelves. Pacific water enters the Chukchi Sea through Bering Strait, and Norwegian Sea water flows onto the Barents shelf over its western edge. All the shelves receive river runoff; see Aagaard and Carmack (1989) for its spatial distribution. Water flows out of the Arctic over the shelves through the Arctic Archipelago in northern Canada. The rest of the exchange between the Arctic and the rest of the world occurs in the only deep connection to the Arctic, Fram Strait, through which the West Spitzbergen Current flows into the Arctic next to Spitzbergen, and the East Greenland Current flows out of the Arctic along Greenland's east coast.

The outflow through Fram Strait is one of two main ways the Arctic Ocean affects global climate (Aagaard and Carmack, 1994). The fresh surface waters and dense deep waters of the Fram Strait outflow in part determine the density profiles of the Greenland, Iceland and Norwegian Seas (the GIN Seas), directly affecting the amount and character of dense water formed in those basins and determining which range of densities will lie at the right depth to overflow to the North Atlantic (Aagaard et al., 1985; Aagaard and Carmack, 1989; Mauritzen, $1996 \mathrm{a}, \mathrm{b})$. The other major role of the Arctic in the global climate is to regulate 
the high-latitude surface heat flux. Heat is transported northward by the global thermohaline circulation, and released to the atmosphere at high latitude. This surface exchange occurs primarily at the margins of the Arctic ocean, especially during the refreezing of the shelves each autumn and through coastal polynyas during the winter. The heat exchange through the surface of the central basins is small by comparison, owing both to the insulating effect of the ice cover, and to the very strong salt stratification of the upper Arctic (Aagaard and Carmack, 1994). Both of these important climate factors, the Fram Strait outflow characteristics, and the surface heat flux, are thus very much determined by the vertical distribution of temperature and salinity in the Arctic Ocean.

The vertical $T / S$ structure of the Arctic is often described as having four layers. At the surface is a mixed layer $30-50 \mathrm{~m}$ thick, at freezing temperature $\left(\approx-1.7^{\circ} \mathrm{C}\right)$ and with very low salinity $(S \approx 31.6) .{ }^{1}$ From 50 to $200 \mathrm{~m}$, the temperature remains near freezing, but the salinity increases to greater than 34.5 , causing this layer to be known as the Arctic halocline. Below $200 \mathrm{~m}$, the potential temperature increases to as much as $0.5^{\circ} \mathrm{C}$ at $500 \mathrm{~m}$ and then decreases slowly with depth to between -0.5 and $-1^{\circ} \mathrm{C}$ at the bottom. The temperature maximum is due to inflow from the Atlantic, thus the warm layer below the halocline (to about $800 \mathrm{~m}$ ) is known as the Atlantic layer. Below $800 \mathrm{~m}$ is known simply as the deep layer.

Salinity increases slowly through the Atlantic layer and the deep Arctic, approaching 35 near the bottom of the basin. At low temperatures, the density depends almost entirely on salinity. This means the Arctic is very strongly stratified in the halocline $\left(\sigma_{\theta}\right.$ increases by $\left.2 \mathrm{~kg} \mathrm{~m}^{-3}\right)$ and very weakly stratified below it ( $\sigma_{\theta}$ increases by less than $0.2 \mathrm{~kg} \mathrm{~m}^{-3}$ below $500 \mathrm{~m}$ ). The strong salt stratification in the upper water column reduces fluid exchange between the surface layer and the Atlantic layer.

\footnotetext{
${ }^{1}$ Salinities are reported on the Practical Salinity Scale 1978 (Lewis, 1980).
} 
This vertical structure appears everywhere in the Arctic basin, with little horizontal variation. Most of the horizontal variability is on the mesoscale: the Arctic is populated with eddies of $10-20 \mathrm{~km}$ radius which appear to be formed at the boundaries (D'Asaro, 1988a, b) but which could also be formed in coastal polynyas (Gawarkiewicz et al., 1998), or under leads in the ice cover (Bush and Woods, 1998). Strong boundary currents have been observed along the shelfbreak and over the central ridges (Aagaard, 1989). There is a weak surface salinity gradient: the surface freshens moving from Fram Strait to Bering Strait. This salinity gradient implies a small variation in freezing point, and therefore in surface temperature as well. Though horizontal gradients are weak, there is a small but noticeable difference in the thermohaline structure between the Canadian and Eurasian basins. The Canadian Basin halocline is somewhat warmer and fresher, but the Atlantic Layer of the Canadian Basin is cooler than that of the Eurasian Basin. These differences in vertical structure, and the exact position of the interface between the Canadian and Eurasian water masses, are discussed further by McLaughlin et al. (1996). Reviews of general Arctic oceanography are provided by Coachman and Aagaard (1974), Carmack (1986), Aagaard (1989), and Schlosser et al. (1995).

The main elements of the Arctic's global role-the character of the outflow, and the distribution of the surface heat flux-are determined by the basins' vertical structure. For example, the Atlantic layer contains more than enough heat to melt the Arctic ice cover (Aagaard and Coachman, 1975). If the halocline were to disappear, the upper layer freshwater flux from the Arctic, the polar albedo, and the distribution of surface heat flux would all undergo drastic changes. The processes maintaining the vertical temperature and salinity distribution in the Arctic are therefore of great interest. 


\subsection{Arctic Shelf-Basin Exchange}

Exchanges between the Arctic shelves and the Arctic basins participate in many aspects of the Arctic system. Through shelf-basin exchange, Atlantic, Pacific, and river water are distributed. Nutrient-rich shelf water is carried to the basin, affecting the Arctic ecosystem (Grebmeier et al., 1997). And off-shelf flows distribute pollutants which have been deposited in the shelf seas, including radioactive waste (Livingston, 1995). In addition to all this, shelf-basin exchange is hypothesized to play a key role in determining the $T / S$ structure of the Arctic basin-an important role indeed, as discussed in the previous section.

The connection between shelf-basin exchange and the basin profiles begins with the process of ice formation. Freezing "distills" fresh water (ice) out of sea water. Ice formed on the shelves eventually moves to the basins, where some of it melts, strengthening the salinity stratification of the upper Arctic and influencing the surface circulation, and the rest remains, contributing to the insulating ice cover until eventually entering the GIN seas through Fram Strait (Aagaard and Carmack, 1989; Steele et al., 1996).

Equally as important as the formation of ice is the attendant formation of dense water, created from the salt rejected during freezing. Shelves may be the preferred site of dense water formation in the Arctic because ice divergence at the coast creates persistent polynyas, allowing greater heat loss to the atmosphere than can occur in the basin, and subsequently, greater ice formation and brine rejection. Further, the shallow depths of the shelves cause the rejected salt to be distributed over a smaller volume. Once formed, the dense water is thought to flow over the shelfbreak, distributing freezing-temperature brine to the halocline and below (Gawarkiewicz et al., 1998).

The halocline and the deep waters appear to require shelf water for their maintenance. The Arctic halocline is not simply a mixture of surface water and water 


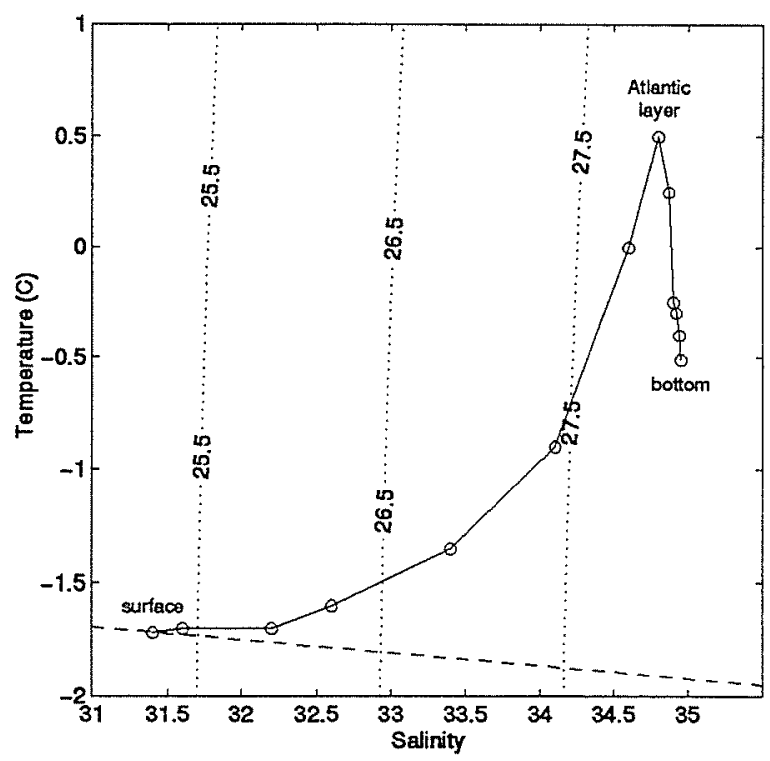

Figure 1.2: Typical Arctic $T / S$ curve. Dotted lines are contours of constant density $\left(\sigma_{\theta}\right.$, in $\left.\mathrm{kg} \mathrm{m}^{-3}\right)$; dashed line marks the freezing point.

from the Atlantic layer, as is evident from a $T / S$ diagram (Figure 1.2). If the halocline were a purely vertical mixture, the $T / S$ curve would lie on a straight line between the surface waters and the Atlantic layer waters. Some cold and salty component is required to pull the curve off the mixing line. Such a component is also required to explain the salinity of the deep Canadian Basin, which near the bottom attains a maximum value in both the vertical and the horizontal (Aagaard, 1981).

No cold, salty region appears in the Arctic hydrography which could supply the missing component by horizontal advection. Therefore, there remain three possible sources for the required mode water: convection in the basins, salt transfer directly from the Atlantic layer, and deep water production on the Arctic shelves. The first, convection driven by brine rejection from freezing in the central Arctic, is unlikely to play a large role since the ice provides a year-round insulating cover, reducing heat transfer to the atmosphere by a factor of $O(100)$ over $98-99 \%$ of the Arctic 
surface area (Smith et al., 1990). Brine rejection does occur from narrow leads which take up $1 \%$ of the Arctic surface, but over the great depths of the basins it is difficult for the salinity to build significantly. Salt transfer from the Atlantic layer, which has a salinity of over 35 as it enters the Arctic through the Fram Strait, has been postulated to occur through double-diffusive processes (Aagaard, 1981; Aagaard et al., 1981). Indications of this process have been observed by Rudels et al. (1994), who conclude that (i) double-diffusive mixing alone cannot explain the vertical structure of the entire basin, and (ii) where it does occur, doublediffusive mixing is not between the Atlantic inflow and the basin water, but rather between the Atlantic inflow and dense water produced on the Barents shelf. Thus the remaining possibility, shelf waters, probably accounts for most if not all of the missing cold, saline mode.

For all these reasons, the shelf-basin exchange is a topic of central importance for understanding both the Arctic system itself, and its role in the global ocean.

\subsection{Previous Work}

Cross-shelfbreak flows are elusive targets of observation. Locating and monitoring dense water formation as it occurs presents myriad technical challenges, and though published observations exist, they are few (Melling and Lewis, 1982; Quadfasel et al., 1988; Melling, 1993; see also Aagaard and Roach, 1990). There have been several estimates of the amount and importance of shelf-basin exchange (Aagaard et al., 1981; Killworth and Smith, 1984; Björk, 1989, 1990; Martin and Cavalieri, 1989; Cavalieri and Martin, 1994; Rudels et al., 1994; Jones et al., 1995). Of these, the studies closest to the present work are those of Killworth and Smith (1984) and Björk (1989). Both are one-dimensional models of the Arctic basin temperature and salinity profiles, which evolve in response to the lateral input of dense water from the Arctic shelves, and to inflows and outflows to and from the basin. Both 
find that dense shelf water input can approximately balance the effects of the inflows and outflows, and maintain the observed basin profiles in steady state. And both make simplifying assumptions which raise some interesting questions:

1. Shelf source. Both studies parameterize the passage over the shelves of runoff and Bering Strait inflow by injecting these sources directly into the basin. In those studies, the shelves' only role is to accept water from the basin surface mixed layer, distill out ice, and return the remaining brine-enriched dense water to the basin. Will accurately representing other sources for the shelves (runoff, Bering Strait, etc.) affect the shelf-basin exchange?

2. Uncertainties. Though both studies vary model parameters within reasonable ranges to find the best fit, neither takes quantitative account of the data uncertainty. Prescribed parameters such as runoff volume and Bering Strait transport are not known perfectly, but only to some level of accuracy. This raises three specific issues:

(a) It is to be expected that inaccuracies in the data could lead to contradictions among the various model equations. An ideal solution will balance these constraints, satisfying those with low uncertainties more closely than those with higher uncertainties.

(b) Even if the model were to capture perfectly the physics of the Arctic, data inaccuracies would prevent the solution from perfectly fitting the data. How much misfit can be ascribed to errors in the data?

(c) Given that there is a range of plausible values for the data (given by the error bars on the data), what is the corresponding range of solutions? In particular, which aspects of the system are well-constrained by the data, and which are very uncertain? 
3. Surface layer exchange. Both models allow dense water from the shelves to enter the basin. But it is equally plausible that water could cross the shelfbreak in the surface mixed layer, driven by the wind. What is the effect of allowing this additional type of shelf-basin exchange? ${ }^{2}$

4. Outflow rate. Both studies parameterize the outflow from the Arctic as a function of the internal density profile using simple rules based on geostrophy. However, Killworth and Smith (1984) find that their approximation fails to capture their assumed Fram Strait velocity structure. Björk (1989) tries several combinations of inflow and outflow rates, and finds that when the Bering Strait inflow is set to its best estimated value (Chapter 3) of $0.8 \mathrm{~Sv}$ [1 Sverdrup $(\mathrm{Sv})=10^{6} \mathrm{~m}^{3} \mathrm{~s}^{-1}$ ] and observed $T / S$ profiles are recovered, the outflow from the Arctic is approximately $1 \mathrm{~Sv}$, or about half the present estimates (see Ch. 3). Can shelf-basin exchange maintain the observed profiles and still supply the observed Arctic outflow?

5. Two-basin structure. Both studies treat the Arctic as one uniform basin. Does resolving the two-basin structure of the Arctic affect the total need for shelf-basin exchange? Are the needs of each basin different?

6. Surface heat flux. Both studies hold the surface mixed layer fixed at the freezing point, regardless of the evolution of the temperature of water entering the mixed layer. Effectively, then, they prescribe that the surface heat flux shall exactly balance the sum of latent heat release on freezing, and the internal oceanic heat flux to the mixed layer. If the true heat flux were to have any other value, a different circulation would be required to maintain

\footnotetext{
${ }^{2}$ One point of view is that when data are sparse and constraints are few, adding degrees of freedom, such as surface mixed layer flow across the shelfbreak, overly complicates the model. However, degrees of freedom such as this one are never truly "left out" of models. They are effectively included and set arbitrarily to zero. Representing them explicitly in the model facilitates exploration of the effects of that choice.
} 
the mixed layer at the freezing point. Given that estimates of the Arctic surface heat flux are very uncertain (Ch. 3), what is the range of circulation adjustment which may be necessary to balance the surface flux?

Killworth and Smith (1984) and Björk (1989) each approach the problem in essentially the same way: A model - that is, the system of possible flows into and out of various levels in the basin -is proposed, and parameters of the model such as the dense water formation rate are prescribed. Ostensibly, the model is then stepped forward in time to find the steady-state $T / S$ structure. In effect, only some of the model parameters are prescribed and held fixed. The rest are "solved for" by tuning them until the resulting $T / S$ structure comes as close as possible to the observed one. The model is considered a success exactly to the extent that the model and observed profiles match. The mismatch, if any, is then analyzed to guide future efforts.

\subsection{Present Work}

The present study proposes to solve the same problem more directly. A box model is formulated, in which the temperature and salinity of each box are prescribed to reflect the observed Arctic $T / S$ profiles. Equations of conservation for mass, heat, and salt are written for each box in terms of the prescribed $T / S$ values, and unknown transports among the boxes. The system is then inverted to find the solution-i.e., the shelf-basin exchange rates and other transports-which comes closest to producing steady state. The solution is then examined to see if it is "close enough" to steady state that departures can be explained by errors in the data.

This approach has several advantages for the present problem. For one, the optimal values of parameters such as shelf-basin exchange are calculated immediately, and do not have to be sought for with many calculations. Another is that 
uncertainties in the data and in the solution are handled naturally, as will be explained in Chapter 2. A third is that the calculation is small, roughly equivalent to inverting a $100 \times 100$ matrix, and therefore can be modified and repeated easily in order to explore the questions posed above.

Solution techniques which incorporate the uncertainty of the problem parameters into the solution method are known as inverse techniques. The application of inverse techniques to oceanography in general is described by Wunsch (1996). These techniques have only recently been applied to the Arctic (Mauritzen, 1996a, b; Thomas et al., 1996), but their foundation on data uncertainties, enabling determination of what is and is not well-constrained by a given data set and choice of model, makes them a natural choice for Arctic problems in which data are sparse and no one physical model is known to be correct.

This study's explicit focus on steady solutions begs the question, why examine the steady problem at all? There are two reasons. First, it is a sound procedure to solve the steady problem as an initial step toward understanding the full, timedependent problem. The Arctic is in steady state to the extent that the effects of the boundary fluxes into and out of the system are balanced by internal processes. The Arctic can be expected to change when this balance does not hold. Understanding such changes will be much easier if the steady balances are clearly identified. Second, the steady problem provides quality control for data on Arctic change. Steady models serve as a null hypothesis. If solutions to the steady problem can be found which, within error bars, are consistent with the available data, then the data are not powerful enough to reject the steady hypothesis. They cannot then be relied upon to resolve and illuminate the processes responsible for Arctic change. The above discussion has shown that previous examinations of the steady shelf-basin exchange have ignored the data and solution uncertainty, simplified the sources supplying the shelves, and omitted potentially important degrees of freedom. The next chapter develops a box model to address these issues. 


\section{Chapter 2}

\section{Box Model Description}

\subsection{Overview}

The model is based on the conservation of mass, heat, salt, and in some calculations, oxygen isotope ratio. Tracer values are prescribed in each of 18 boxes, which represent the basins and their adjoining shelves (Figure 2.1). The model is forced by boundary fluxes: inflows carry mass and tracers into various boxes, while outflows remove them. Further, fresh water is removed from the surface boxes to represent the formation and export of ice, tending to increase the salinity of the surface boxes. To conserve mass and tracers in each box, there must be an internal circulation among the boxes which redistributes the mass and tracer anomalies introduced by the boundary forcing.

The internal circulation which maintains a conservative steady state is the unknown for which the model is solved. Each transport from one box to another is a separate component of the solution. In the basins, transport is allowed from each box to the boxes above and below it, and to the box at the same level in the other

basin (except for the deepest boxes, separated by the Lomonosov Ridge). Basin flows carry the average properties of the originating box, and accordingly, each 

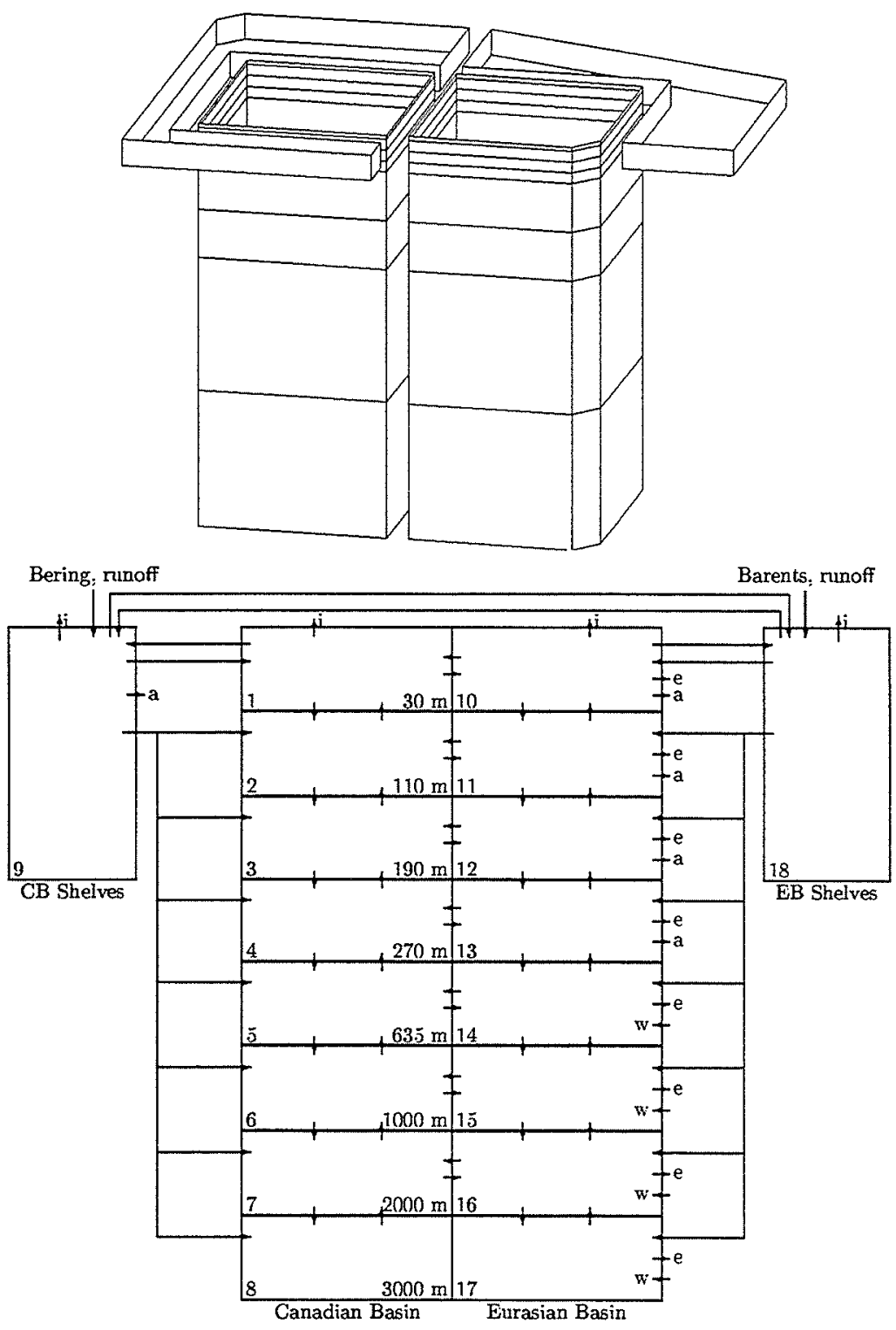

Figure 2.1: Box model. Above: approximate physical arrangement of the boxes. Below: schematic diagram of the model. Box numbers are indicated in the lower left of each box; depths at the bottoms of the basin boxes are also given. Arrows indicate unknown transports. Water removed as ice is indicated by $i$, outflows through the Arctic Archipelago and East Greenland Current by a and e, and West Spitzbergen Current inflows by w. 
unknown transport is required to be positive. Equal transport in each direction between two boxes is analogous to large-scale diffusion, i.e., down-gradient transport of tracers with no net advection of volume. Imbalance in the two directions of transport represents net advection between boxes.

The shelf boxes produce a wider range of water types than do the basin boxes. Flows from one shelf box to the other, and flow from the Canadian Basin shelves out the Arctic Archipelago, carry the average shelf properties. Flow to the basin surface layer carries the average properties not of the entire shelf, but of the top $30 \mathrm{~m}$, representing the assumption that this transport takes place via the winddriven mixed layer. Dense overflows have freezing temperature, and the salinity of the destination box: at low temperatures, density is controlled primarily by salinity, so this choice represents the sinking of shelf water to its density level.

Ice formed in the surface boxes - shelf and basin-is removed directly from the system. The simplicity of this representation is justified by the result that within error bars, net ice formation equals net ice advection in all regions of the Arctic (Thomas et al., 1996). (An elaboration of the box model which includes the possibility of ice melt, and advection of ice among the surface boxes, is considered in Section 4.4.6.) Since the internal energy of ice is so low relative to liquid water, an effective transport potential temperature for ice is estimated by dividing the internal energy of sea ice (relative to sea water at $0^{\circ} \mathrm{C}$ ) by the liquid density and heat capacity $\left(4 \times 10^{6} \mathrm{~J} \mathrm{~m}^{-3} \mathrm{~K}^{-1}\right)$, yielding $t=-83.7^{\circ} \mathrm{C}$. In other words, a flux of liquid water at $-83.7^{\circ} \mathrm{C}$ (were it possible) would have the same heat transport as an ice flux of the same mass. The exiting ice is assumed to have a salinity of $S=3$. This is a gross simplification, as the mechanisms determining net brine rejection over a season of ice melt and growth are complicated (Maykut, 1985), but the overall brine rejection produced by removing the net ice at $S=3$ is within the error bars of the salt fluxes estimated by Thomas et al. (1996) with a more complete model. 
The boundary forcing values are prescribed, based on published estimates of the mean transports of Arctic inflows and outflows. A complication arises because some of the boundary fluxes affect more than one box. For example, the density range of the West Spitzbergen Current (WSC) implies that it enters the Arctic below the halocline, ${ }^{1}$ and therefore enters boxes 14-17 (Figure 2.1). While the total mass, heat, and salt inputs of the WSC are known (Chapter 3), their distribution with depth is not clearly constrained. This is handled in the model by making the WSC input to each of those four boxes an unknown, and then constraining the total of those four unknowns to match the total estimated WSC input. Details of how this is done for various boundary fluxes, including the WSC, the East Greenland Current, the Arctic Archipelago outflow, and the total ice formation, are given in Section 2.6 below.

The model equations are statements for each box of the steady conservation of volume (as an approximation for mass), potential temperature (as an approximation for heat), and salt, plus the constraints on the boundary forcing described in the preceding paragraph. The full system of equations and unknowns is listed in Table 2.1. Each element of the solution (i.e., each transport) is additionally constrained to be positive.

The model is summarized by the matrix system

$$
\begin{aligned}
& E \mathbf{q}+\mathbf{f}+\mathbf{n}=\mathbf{0} \\
& \mathbf{q} \geq 0 .
\end{aligned}
$$

Here $\mathbf{E}$ is a coefficient matrix, and $\mathbf{f}$ is the vector of mass, temperature, and salt forcing due to the boundary fluxes. The circulation $\mathbf{q}$ is determined as the leastsquares solution to (2.1), with the norm of the residual vector $\mathbf{n}$ to be minimized, subject to (2.2). If the residuals with minimum norm are commensurate with

\footnotetext{
${ }^{1}$ See Section 4.4.3 for discussion of an alternate hypothesis.
} 
Table 2.1: Model constraints and unknowns. Conservation of $\delta^{18} \mathrm{O}$ in boxes $1-18$ adds an additional 18 constraints.

\begin{tabular}{clcl}
\hline Index & \multicolumn{1}{c}{ Equation } & Index & \multicolumn{1}{c}{ Unknown } \\
\hline $1-18$ & Volume conservation, boxes 1-18 & $1-14$ & Dense shelf water flows \\
$19-36$ & Potential temperature conservation, boxes 1-18 & $15-18$ & Shelf-surface mixed layer exchange \\
$37-54$ & Salt conservation, boxes 1-18 & $19-20$ & Inter-shelf exchange \\
$55-57$ & Arctic Archipelago volume, temperature, and salt fluxes & $21-34$ & Inter-basin exchanges \\
58 & Arctic Archipelago Canadian/Eurasian Basin distribution & $35-62$ & Vertical exchanges \\
59 & Total ice formation & $63-67$ & Outflows to AA \\
$60-62$ & EGC Polar Water volume, temperature, and salt fluxes & $68-71$ & Outputs as ice \\
$63-65$ & EGC Atlantic Water volume, temperature, and salt fluxes & $72-79$ & Outflows to EGC \\
$66-68$ & EGC Deep Water volume, temperature, and salt fluxes & $80-83$ & Inputs from WSC \\
69 & WSC Atlantic Water volume flux & & \\
70 & WSC Deep Water volume flux & & \\
\hline
\end{tabular}

the uncertainties of the equations, then the solution is consistent with the data and with the conservation statements; otherwise, a consistent steady solution does not exist. The following sections describe the details of the model system and its analysis.

\section{$2.2 \quad$ Structure}

The 18 boxes of the model are distributed as follows (Figure 2.1): eight layers in each of the Canadian and Eurasian Basins ${ }^{2}$ plus one box for each basin's surrounding shelves. Further subdivision in the horizontal is impractical: given the weak horizontal gradients in the Arctic and the poor data coverage, it is difficult to resolve differences on smaller scales. In the vertical, the basins are partitioned as follows: the surface mixed layer box represents the upper $30 \mathrm{~m}$. The halocline extends to $270 \mathrm{~m}$ depth and is divided into three boxes of $80 \mathrm{~m}$ each. The Atlantic layer extends from 270 to $1000 \mathrm{~m}$ depth, divided into two boxes of $365 \mathrm{~m}$ each. The deep Arctic extends from $1000 \mathrm{~m}$ to the bottom, taken here to be $3000 \mathrm{~m}$,

\footnotetext{
${ }^{2}$ McLaughlin et al. (1996) have suggested that the most appropriate dividing line between the Atlantic and Pacific water masses may be closer to the Alpha Ridge than to the Lomonosov Ridge. Little changes in the model if the horizontal partitioning is interpreted in this other way.
} 
and is spanned by two boxes of $1000 \mathrm{~m}$ each. Increasing the vertical resolution by adding a few boxes does not affect the model results; adding many boxes can not be supported by the vertical resolution of the available data.

If fluid mixes along isopycnals, then identifying the boxes with fixed depth levels, instead of density-defined layers, may lead to errors in the representation of the horizontal fluxes between basins. Such errors are likely to be small, however: over the relatively large depth interval of each level, the ranges of densities in each basin overlap substantially.

\subsection{Temperature and Salinity}

The prescribed potential temperatures and salinities of the boxes are based on data from the World Ocean Atlas 1994 (NODC, 1994). ${ }^{3}$ Annual average temperature and salinity profiles for five-degree boxes from the atlas were averaged together at the atlas standard depths for each basin and for the shelves. Standard routines (Fofonoff, 1977) were used to calculate potential temperatures. Linear interpolation between the standard depths produced the profiles used in the model; the profiles were then averaged over the depth range of each box to give the final box values. The potential temperatures and salinities for each box are shown in Figure 2.2.

With the exception of freshwater, Arctic salinities fall in the numerically narrow range of 30-35. Since these values are the coefficients of the salinity conservation equations, the narrow range may restrict the linear independence of those equations. Using salinity anomalies, measured from a median value of 34.4 , for the calculations modestly increases the linear independence, increasing the numerical

\footnotetext{
${ }^{3}$ The spatial resolution of this data set varies a great deal. For certain, small-scale features such as boundary currents are not clearly resolved. Points that were not clearly either on the shelf or off-that is, those near the shelf break-were omitted, and therefore few profiles through boundary currents are likely to appear in the averages. The more recent Joint U.S.-Russian Atlas (Gore-Chernomyrdin Commission Environmental Working Group, 1997) has very much better coverage but at this time only the winter fields are available.
} 


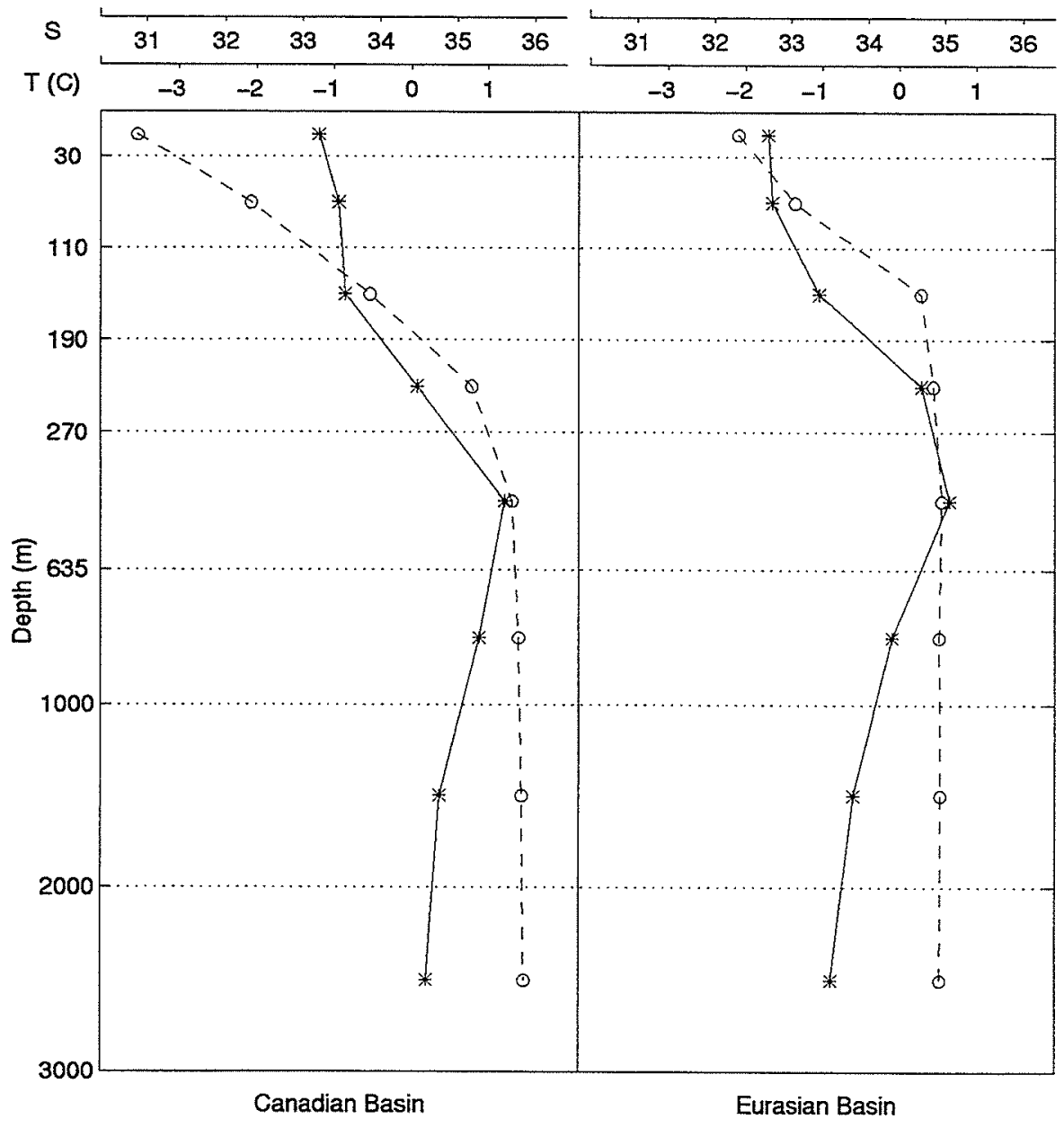

Figure 2.2: Prescribed potential temperatures (solid curves) and salinities (dashed curves) for each box. Vertical scale is distorted. 
stability of the matrix inversions. By coincidence, zero is close to the median value for temperature and $\delta^{18} \mathrm{O}$, so a similar translation is not used for those tracers.

The choice was made to postulate the temperature and salinity and infer the circulation because in the Arctic, temperature and salinity are better known than the circulation. The sensitivity of the results to the prescribed temperatures and salinities is described in Section 4.2.4.

\subsection{Oxygen Isotope Ratio}

As will be shown in Chapter 4, it is desirable to consider another tracer in addition to temperature and salinity. Many of the model calculations will therefore include the oxygen isotope ratio ${ }^{18} \mathrm{O} /{ }^{16} \mathrm{O}$. This ratio is generally expressed as the per mil deviation, $\delta^{18} \mathrm{O}$, from a standard (Bauch, 1995). Its usefulness as an Arctic tracer (Östlund and Hut, 1984; Östlund et al., 1987; Schlosser et al., 1994; Bauch et al., 1995) stems first from the strong $\delta^{18} \mathrm{O}$ signal of runoff $(-21.0 \%)$ relative to all other water types $\left(-2.0\right.$ to $0.3 \%$ ), and second from the fact that the $\delta^{18} \mathrm{O}$ value of a water parcel can be changed only by fractionation due to evaporation, which preferentially removes the lighter isotope, or freezing, which preferentially removes the heavier. ${ }^{4}$ A strong difference in surface $\delta^{18} \mathrm{O}$ values exists between the shelves and basins, with lower $\delta^{18} \mathrm{O}$ in the basins. Because direct evaporation is minimal in the Arctic, (SCOR WG-58, 1979), and freezing only changes the $\delta^{18} \mathrm{O}$ value by approximately $10 \%$ of the ocean-runoff difference (Schlosser et al., 1994), only runoff can explain the observed decrease in surface $\delta^{18} \mathrm{O}$ values toward the center

\footnotetext{
${ }^{4}$ Because ${ }^{18} \mathrm{O} /{ }^{16} \mathrm{O}$ is a ratio of concentrations, it is not obvious that it is a conservative quantity. However, it is easily shown that the ratio of two concentrations $C_{A} / C_{B}$ is conserved on mixing to the extent that $C_{B}$ is a constant. In the present case, $C_{B}$ represents the concentration of ${ }^{16} \mathrm{O}$ (or more precisely, $\mathrm{H}_{2}{ }^{16} \mathrm{O}$ ) in sea water. Typically, of every 10,000 water molecules, 9,976 are $\mathrm{H}_{2}{ }^{16} \mathrm{O}$, and 20 are $\mathrm{H}_{2}{ }^{18} \mathrm{O}$. (The remaining four are $\mathrm{H}_{2}{ }^{17} \mathrm{O}$. Bauch, 1995, p.23.) A change in the ${ }^{18} \mathrm{O} /{ }^{16} \mathrm{O}$ ratio of $20 \%$ implies that the concentration of ${ }^{16} \mathrm{O}$ itself varies by about $20 \%$, or $2 \%$. In other words, it is nearly constant. Thus, ${ }^{18} \mathrm{O} /{ }^{16} \mathrm{O}$-and $\delta^{18} \mathrm{O}$-are conserved on mixing in the Arctic to approximately $2 \%$.
} 
of the Arctic Ocean (Bauch et al., 1995). This decrease is surprising, since the source of low- $\delta^{18} \mathrm{O}$ water-runoff-feeds the shelves, not the basins. Runoff must somehow make its way somewhat coherently from the shelves to the center. (This idea is elaborated in Section 4.4.1.) In addition, slightly reduced $\delta^{18} \mathrm{O}$ values in the deep Eurasian Basin indicate the presence of river-fed shelf water at depth (Bauch et al., 1995). The ${ }^{18} \mathrm{O} /{ }^{16} \mathrm{O}$ ratio is therefore a promising source of information about shelf-basin exchange.

The available $\delta^{18} \mathrm{O}$ data for the Eurasian Basin (Bauch, 1995) comes from approximately 30 stations in the basin and approximately 20 stations on the Barents and Laptev shelves. The available data for the Canadian Basin are fewer yet, consisting of one station from Bauch (1995) and three from Östlund et al. (1987). Indirect estimates of the Canadian Basin shelf values are provided by Bauch (1995, Tables 12 and 15). The sparsity of the data makes an average profile impossible to estimate meaningfully; instead, representative profiles are crudely constructed from what is available. "Low" and "high" profiles are also constructed which represent the variation in the data, most of which occurs in the upper 100 or $200 \mathrm{~m} .^{5}$ These are used in determining the sensitivity of the results to the assumed profiles (see Section 4.3 .4 below). The profiles of $\delta^{18} \mathrm{O}$ used in the model are plotted in Figure 2.3. The $\delta^{18} \mathrm{O}$ values for the shelves, and the transport $\delta^{18} \mathrm{O}$ values of the boundary fluxes are also taken from Bauch (1995) and are listed in Table 2.2.

The inclusion of $\delta^{18} \mathrm{O}$ allows for additional model constraints, requiring that $\delta^{18} \mathrm{O}$ be conserved in each box. The fluxes into the Arctic of $\delta^{18} \mathrm{O}$ are computed by the same procedure as are the fluxes of potential temperature and salt (Section 3.4.2, below.) No constraints are placed on the total outflowing $\delta^{18} \mathrm{O}$ flux in any given current, since there are no applicable data available.

\footnotetext{
${ }^{5}$ Data from the Canadian Basin are expected to be available in the near future (B. Ekwurzel, personal communication, 1997). In the meantime, the ranges used here seem to cover the range of $\delta^{18} \mathrm{O}$ values in the data which has been taken but not yet made available (P. Schlosser, personal communication, 1997).
} 


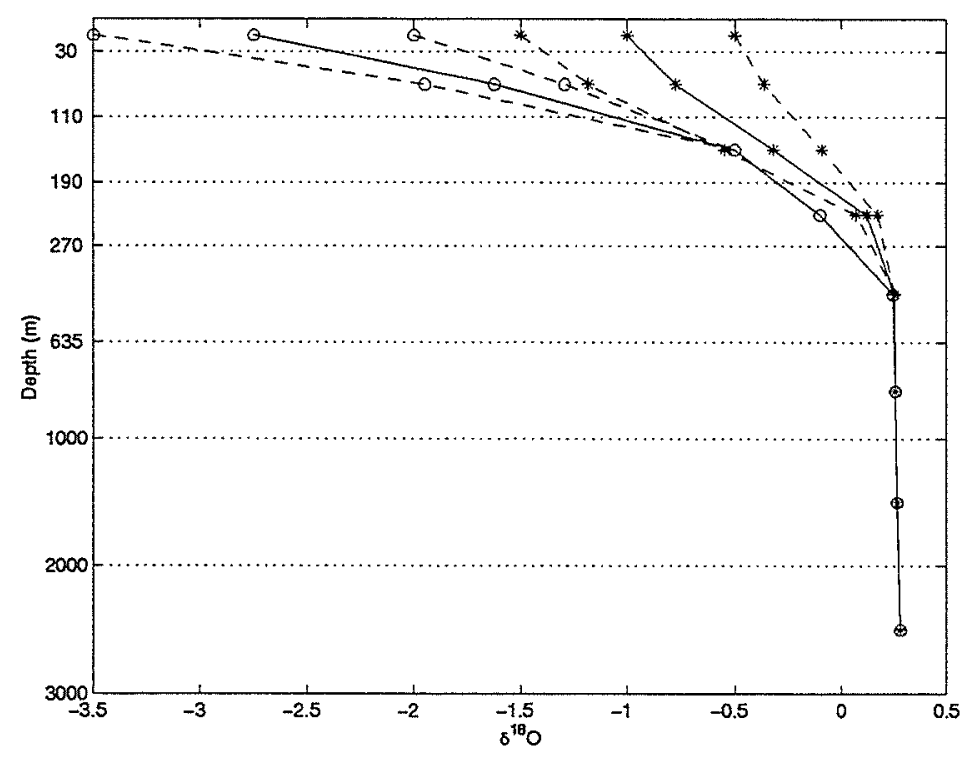

Figure 2.3: Representative $\delta^{18} \mathrm{O}$ profiles for the Canadian (o) and Eurasian (*) basins. Solid lines indicate the standard profiles; dotted lines are the variations used to assess sensitivity (see Section 4.3.4).

Table 2.2: Shelf $\delta^{18} \mathrm{O}$ and boundary flux transport $\delta^{18} \mathrm{O}$.

\begin{tabular}{ll}
\hline & $\delta^{18} \mathrm{O}(\%)$ \\
\hline Canadain Basin & -1.91 \\
Eurasian Basin & -0.10 \\
Bering Strait & $-1.0 \pm 0.5$ \\
Runoff & $-21.0 \pm 1.0$ \\
West Spitzbergen Current & $0.324 \pm 0.005^{a}$ \\
Barents Inflow & $0.2 \pm 0.1^{b}$ \\
Ice & surface value $+2.1^{c} \pm 0.5^{d}$ \\
\hline
\end{tabular}

${ }^{a}$ Arbitrary uncertainty.

${ }^{b}$ Lowered from the Atlantic value to reflect river input from Norway (Blindheim, 1989).

${ }^{c}$ For fractionation.

${ }^{d}$ Arbitrary uncertainty. 


\subsection{Uncertainty}

Each model equation contains errors whose values are by definition unknown. Estimates of their likely sizes and correlations are required to judge whether the size and correlations of the solution residuals can be explained by the errors.

The errors are of two types. The first type is errors in the statements themselves, such as those introduced by use of a temperature conservation statement when energy is in fact conserved. To recognize such errors, known as model errors, the volume conservation statements for each box are assumed to be accurate to $\pm 0.02 \mathrm{~Sv}$, and the potential temperature and salinity conservation statements are assumed accurate to $\pm 0.03 \mathrm{~Sv}^{\circ} \mathrm{C}$ or $\mathrm{Sv} \cdot \mathrm{psu}$, respectively. The uncertainty of the $\delta^{18} \mathrm{O}$ conservation statements is set to $\pm 0.1 \times 10^{3} \mathrm{~m}^{3} \mathrm{~s}^{-1}$, i.e., $0.1 \mathrm{~Sv}$ multiplied by $1 \%$. These errors are assumed uncorrelated. Their values are chosen as orderof-magnitude estimates of the minimum plausible error: the difference between volume and mass conservation is approximately $2 \%$, multiplied by flows on the order of $1 \mathrm{~Sv}$ yields $0.02 \mathrm{~Sv}$ uncertainty. This value is augmented for the tracer conservations to acknowledge small errors in the prescribed tracer values in the boxes. The sensitivity of the solution to these choices is evaluated in Section 4.4.8.

The second type includes errors in the data. For example, one term in the mass budget for the Canadian Basin shelf box is the Bering Strait input; errors in the estimate of average Bering Strait transport increase the uncertainty of the mass conservation statement. The treatment of forcing data errors in the model is discussed in the next section.

\section{$2.6 \quad$ Forcing}

Estimates of the boundary fluxes which force the model are derived in Chapter 3 . The derivation of the estimates provides not only the flux values, but also estimates 
of their uncertainties and error correlations. Each flux (volume, potential temperature, salt, $\delta^{18} \mathrm{O}$ ) affects the corresponding budget equation of the box or boxes it enters or exits. As each flux is mapped to its appropriate equation, the covariances between flux estimate errors are mapped into covariances between errors in the equations. In this way the error structure of the model system is determined.

Some of the boundary fluxes affect more than one box, as was mentioned above. The details of assigning each forcing term to its equation or equations depends on how many boxes the flux affects. The fluxes fall into three groups: fluxes affecting just one box, outflows affecting more than one box, and inflows affecting more than one box.

\subsubsection{Forcing affecting one box}

Fluxes which affect just one box are the simplest to treat. The fluxes are included directly in the forcing vector $\mathbf{f}$ of (2.1). The uncertainties, and the covariance with other equations, are accordingly augmented. Fluxes in this group are those through the Bering Strait and those into the Barents Sea, as well as the surface heat flux from the shelf polynyas and from the ice-free region of the Barents Sea. In addition, river runoff is very nearly evenly split between the two basins (Aagaard and Carmack, 1989), so the Canadian Basin shelf box and the Eurasian Basin shelf box each receive a flux of freshwater equal to $1 / 2$ the total. The variances and covariances associated with those fluxes are set to $1 / 2$ those of the total flux. The "Other Shelf" and "Basin" surface heat fluxes are similarly split between the two shelf boxes and between the two basin surface boxes, respectively.

\subsubsection{Forcing affecting more than one box: outflows}

Outflows which originate from more than one box are accounted for by letting the outflow from each of the relevant boxes be unknown. Those unknowns are 
constrained by three additional equations appended to the system, respectively stating that their combined transport, potential temperature transport, and salt transport should be equal to the estimated boundary flux values.

Fluxes in this category are those of the East Greenland Current (EGC), ice formation, and the Arctic Archipelago outflow. The EGC Polar Water exits from the upper four Eurasian Basin boxes, the Atlantic Water from the next two, and the Deep Water from the lowest two.

It is conventionally assumed that the the Arctic Archipelago (AA) outflow is drawn from the Canadian basin, but to my knowledge no evidence exists which points to any particular source for this outflow. The topography (Figure 1.1) suggests to me that transports through Jones and Lancaster sound originate on the shelf system of the Archipelago itself, while the Nares Strait flow stems directly from the Eurasian Basin, and that impression has dictated the model design. In the model, the AA outflow comes from the Canadian Basin shelf box, and from the upper four boxes of the Eurasian Basin, since the AA sill depth is $250 \mathrm{~m}$ (Rudels, 1987). The effect of this representation of the AA outflow on the model results is discussed in Section 4.4.2. The flow through Jones and Lancaster Sounds appears to be 1.5-4.5 times as large as that through Nares Strait (Addison, 1987; Fissel et al., 1988), so an additional equation is appended to the system stating that the contribution from the Canadian Shelf is three times the total from the Eurasian Basin, with a standard error of $1.4 \mathrm{~Sv}$. The error in this equation is assumed uncorrelated to those in other equations.

The net ice formation in each of the four surface boxes are model unknowns. They are constrained by an appended equation stating that the total ice growth must equal the estimated Fram Strait ice export, an assumption loosely justified by Thomas et al.'s (1996) result that total net ice formation is equal to the total export, and by the observation that nearly all ice exiting the Arctic leaves through Fram Strait. (A more complete ice model, including ice melt and ice advection 
within the Arctic, is used in selected calculations. See Section 4.4 .6 below.) No constraints are placed on the total temperature or salt flux carried by the ice; the relative uncertainties of the transport temperature and salinity are so small compared to the relative uncertainty of the volume flux that heat or salt flux constraints would be completely redundant.

\subsubsection{Forcing affecting more than one box: inflows}

The West Spitzbergen Current is represented in the model as entering the four deepest boxes of the Eurasian Basin: the upper two receive the Atlantic Water and the lower two the Deep Water. This parameterizes the diving of the incoming Atlantic Water (AW) under the more buoyant halocline, as was done by Killworth and Smith (1984). Rudels et al. (1996) have suggested that this representation may be inaccurate; that possibility is explored in Section 4.4.3. The volume transports into each box are unknowns, constrained again by an additional equation stipulating that their sum be equal to the estimated total flux.

These unknown transports are each assumed to carry the transport potential temperature and salinity (and $\delta^{18} \mathrm{O}$ as appropriate) estimated in Chapter 3. A problem with this choice is that the uncertainty in the temperature and salt fluxes due to errors in the transport temperature and salinity estimates is not taken into account. The influence of errors in the transport temperature and salinity is significant in the case of the WSC Atlantic Water heat flux (Chapter 3). As a partial remedy, the uncertainty of the temperature and salt conservation equations for the affected boxes is increased. Specifically, their variance and their covariance with the other equations is augmented in the simplest way possible such that the sum of the equations for the affected boxes has the error structure given by the flux estimates. Thus the uncertainty of the forcing is increased in an attempt to account for what is in fact error in the equation coefficients. This technique has 
been known to introduce bias into the model solution. For more information and further references see Section 5.5 of Wunsch (1996).

\subsection{Solution Methods}

\subsubsection{Scaling}

Prior to analysis the system is scaled in three ways. First, care must be taken that arbitrary differences in the magnitudes of the coefficients (such as those due to definitions of measurement units) do not put unnatural numerical weight on one equation over another. To avoid this, the system is row-scaled, i.e., each equation is divided by the norm of the vector of its coefficients. (The analogous procedure of column scaling is not used, since the difference in coefficients from one variable to another represent physical differences in temperature or salinity.) Next, the equations are scaled by the expected variance-covariance matrix of the errors, to rotate them into the canonical system of independent equations with equal variance for which the standard least-squares derivation is valid. Finally, the unknowns are scaled by prior assumptions of their likely size, so that expected differences in magnitude will be accounted for in choosing the solution of minimum size. (In practice this third scaling makes no difference, because the prior assumption of the size of all unknowns is set to the same value of $1 \mathrm{~Sv}$.) Scaling in least squares problems is discussed in detail in Section 3.3 of Wunsch (1996).

\subsubsection{Rank determination}

The complete system, with $\delta^{18} \mathrm{O}$, amounts to 88 constraints on 83 unknowns (Table 2.1). To assess the possibility of numerical instability, the singular values of 


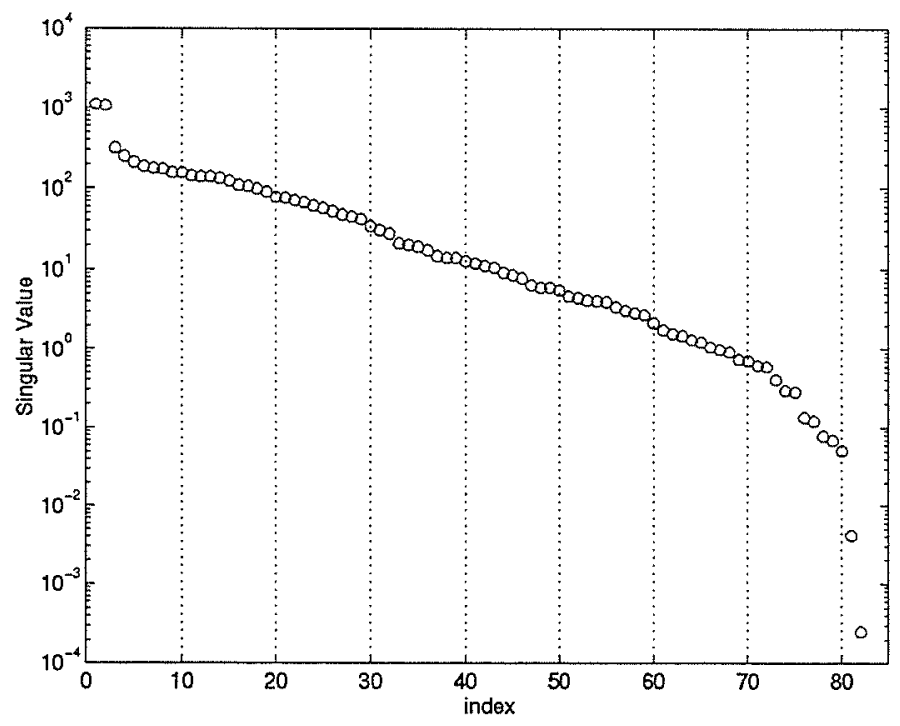

Figure 2.4: Singular values of the scaled 88 -by-83 system matrix $\mathrm{E}$, with $\delta^{18} \mathrm{O}$ constraints, after scaling. The $83 \mathrm{rd}$ singular value (not shown) is less than $8 \times 10^{-15}$.

the scaled system were calculated. The maximum reasonable choice of $\mathrm{rank}^{6}$ for this system is $82:$ the 83 rd singular value (as computed by MATLAB) is less than $8 \times 10^{-15}$, small enough to be attributed entirely to computational noise (Figure 2.4). Computations were performed with the system rank truncated to 80 , and to 75 , with no appreciable difference in the solutions or their uncertainty. When $\delta^{18} \mathrm{O}$ constraints are not included, the system has 70 constraints on 83 unknowns, and the maximum choice for the rank is 65 (not shown). This system was also tested for sensitivity to truncation, and none was found. Usually in linear systems, both the solution and especially the uncertainty are highly sensitive to the choice of rank, so this result is surprising. The explanation seems to be that the uncertainty is strongly suppressed by the non-negative constraints. This point is

\footnotetext{
${ }^{6}$ In linear systems, there is a trade-off between resolution and uncertainty, which can be controlled by removing unstable structures in the system associated with small singular values, or "truncating" the system. See Wunsch (1996) for a complete discussion.
} 
discussed further in the following section.

\subsubsection{Optimal solutions: non-negative least squares and Monte Carlo}

Once the scaled system is determined, the Non-Negative Least Squares (NNLS) algorithm (Lawson and Hanson, 1974) is used to determine a non-negative solution $\tilde{\mathbf{q}}$ which minimizes the residual norm.

The uncertainty of $\tilde{\mathbf{q}}$ (the solution uncertainty) is of two types: uncertainty arising from model and data errors, and nullspace uncertainty. The former is due to the errors discussed in Section 2.5 above: since the model equations and forcing terms are not exact, but only close to their true values, the calculated value of $\tilde{\mathbf{q}}$ is also only close to its true value. The solution uncertainty measures the likely size of the error. Since the NNLS procedure is non-linear, no general, tractable, closed-form expression for the solution uncertainty exists (see Appendix A for a discussion). Instead the uncertainty is estimated by Monte Carlo simulation. In these simulations, 1000 realizations are made of a random perturbation vector, whose elements are uncorrelated Gaussian variables with zero mean and unit variance. Each realization is added to the forcing vector $\mathbf{f}$, and $\tilde{\mathbf{q}}$ is recomputed, generating 1000 realizations of the random variable $\tilde{\mathbf{q}}$. Estimates of the statistics of $\tilde{\mathbf{q}}$, such as modes and confidence intervals, are then calculated from the sample. Note that while the noise is Gaussian, $\tilde{\mathbf{q}}$ is not, owing to the non-linearity of the NNLS operator.

The nullspace uncertainty stems from the rank deficiency of the system of equations. The rank deficiency implies that there could be many solutions which minimize the residual. These can be expressed as a particular solution, plus an unknown nullspace contribution which does not affect the residual norm. ${ }^{7}$ Usu-

\footnotetext{
${ }^{7}$ In underdetermined systems, the solution nullspace comprises those aspects of the solution
} 
ally, little can be said regarding the nullspace uncertainty, since by definition it is comprised of those structures in the solution which the model is not capable of constraining. Adding any linear combination $\mathbf{N x}$ of the nullspace vectors (where $\mathbf{x}$ is an arbitrary vector of coefficients) to the optimal solution $\tilde{\mathbf{q}}$ produces the same residual as did $\tilde{\mathbf{q}}$. In the present case, however, the non-negative requirements create an exception.

The non-negative constraints, $\tilde{\mathbf{q}}+\mathbf{N x} \geq 0$, yield a condition on permissible nullspace contributions,

$$
\mathrm{Nx} \geq-\tilde{\mathbf{q}}
$$

The extent to which this limits the nullspace uncertainty depends on the structure of the nullspace. The question is, what is the maximum possible value of $\|\mathbf{N x}\|$ subject to (2.3)? If it is unbounded, the nullspace uncertainty is also unbounded. If it is bounded, then so is the nullspace uncertainty. And if, due to the nature of $N$ and $\tilde{\mathbf{q}}$, there is no non-zero $x$ which satisfies (2.3), then the nullspace uncertainty is zero. The last case is the one which applies for the model systems considered in this study.

This is shown as follows. Since $\mathbf{N}$ is orthogonal, $\|\mathbf{N x}\|=\|\mathbf{x}\|$. Therefore, the maximum value of $\|\mathbf{x}\|$ subject to (2.3) is the maximum norm of allowable nullspace contributions, or the size of the nullspace uncertainty. The maximum which the model cannot constrain. For example, if a model states only that two unknowns sum to a constant,

$$
x+y=c
$$

then the solution can be written

$$
\left(\begin{array}{l}
x \\
y
\end{array}\right)=\frac{c}{2}\left(\begin{array}{l}
1 \\
1
\end{array}\right)+\frac{a}{2}\left(\begin{array}{c}
1 \\
-1
\end{array}\right)
$$

where $a$ is unknown, and unknowable without additional information. In this case, the nullspace comprises all multiples of the vector $\left(\begin{array}{c}1 \\ -1\end{array}\right)$. The sum $c$ of the unknowns is constrained by the model, but the difference $a$ between them is in the nullspace. For any linear model, the nullspace is orthogonal to all constrained components of the solution. For detailed discussions, see Strang (1993) and Wunsch (1996). 
of $\|\mathbf{x}\|$ subject to (2.3) can be determined using standard quadratic programming routines (e.g., Grace, 1990). This has been done for all of the systems in this thesis, and in every case, the maximum value of $\|\mathbf{x}\|$ subject to (2.3) is 0 . In other words, the non-negative constraints entirely eliminate the nullspace uncertainty, and the non-negative solution of minimum residual norm is unique. ${ }^{8}$

This result provides some insight to why the calculations proved insensitive to rank truncation. Apparently, the singular vectors associated with small-tovanishing singular values are such that they cannot be added to the NNLS solution without driving some solution elements negative. For this reason, vectors associated with small singular vectors were not used in the untruncated calculations; and thus, making them unavailable to the solution by truncation made no difference.

\subsubsection{Extreme solutions: linear programming}

The optimal solutions given by the NNLS operator are those which best satisfy the constraints of conservation and the flux estimates. They answer the question, "What solution best fits the data?" Other questions of interest are, "How much dense water can be produced without violating the constraints too much? What is the least amount of shelf basin exchange still consistent with the data within error bars?" These solutions, which I will refer to as extreme solutions, minimize or maximize some aspect of the solution, subject to maintaining acceptable--but not minimal-residuals.

\footnotetext{
${ }^{8}$ This happy result, true for the systems in this thesis, is by no means guaranteed in general. In systems where nullspace uncertainty persists in spite of non-negative constraints, a common procedure is to select the solution of minimum norm, i.e., that with the least nullspace contribution. For systems such as those considered here, where the unknowns represent transports, such a choice would represent the solution of minimum total transport. That solution is easily determined. From the NNLS solution $\mathbf{q}_{N N I S}$, the nullspace contribution $\mathbf{N}^{\top} \mathbf{q}_{N N I S}$ is subtracted to get $\tilde{\mathbf{q}}$. If the result is negative, the Least Distance Programming algorithm (Lawson and Hanson, 1974 ) is used to determine the smallest possible vector in $\{\mathbf{N}\}$ which when added back to $\tilde{\mathbf{q}}$ will restore it to non-negativity. Similar techniques have been used previously (Fu, 1981; Tziperman and Hecht, 1987; see also Wunsch, 1996, Chapter 5).
} 
Such solutions are found using the method of linear programming, in which a linear combination $\mathbf{a}^{\mathrm{T}} \mathbf{q}$ of the unknowns is minimized subject to arbitrary constraints Aq $\leq$ b. Section 5.2 of Wunsch (1996) gives a brief explanation and further references. In this study, the inequality constraints will be used to enforce non-negativity, and also to restrict the absolute values of the residuals to 1.5 standard errors. Subject to those constraints, minimum and maximum values of three solution aspects will be sought:

1. Dense water formation: the sum of the fourteen transports (seven in each basin) from the shelves to the sub-surface boxes.

2. Surface mixed layer exchange: the sum of the four surface layer transports over the shelfbreak: (i) Canadian Basin to shelf, (ii) shelf to Canadian Basin, (iii) Eurasian Basin to shelf, (iv) shelf to Eurasian Basin.

3. Shelfbreak upwelling: Flows of saline water onto the shelf will be considered in Section 4.4.5.

Before these techniques can be used to find model solutions, it is necessary to estimate the transports of the fluxes across the boundary of the Arctic system. This is the subject of the next chapter. 


\section{Chapter 3}

\section{Boundary Fluxes}

\subsection{Introduction}

Arctic budgets are commonplace, and for good reason. Surveying the ice-covered Arctic interior has long been exceptionally difficult and remains challenging even with today's submarines and icebreakers. By comparison, the marginal flows into and out of the Arctic are both accessible and spatially concentrated: exchange occurs only through the Fram Strait, the Bering Strait, the Arctic Archipelago, and over the western edge of the Barents Sea (Figure 1.1). It is natural, then, to learn what one can by budgeting the fluxes through these connections. Such budgets may be used to evaluate the Arctic's role in global processes, to rank the relative importance of the various exchanges to a given problem, or as boundary conditions for models of the interior.

The most frequently cited Arctic budgets are those of Aagaard and Greisman (1975) and Rudels (1987). Since these were published, several estimates of individual fluxes have appeared (cited below), and one purpose of this chapter is to include these recent results in the Arctic budgets. Missing from nearly all published flux estimates, however, are estimates of their uncertainties; most of the few exceptions 
provide ad hoc error bars, without quantitative basis. The main purpose of the chapter, therefore, is to introduce a quantitative discussion of uncertainty to the Arctic mass, heat, and salt budgets.

The lack of rigorous uncertainty estimates in previous work is understandable: determining the error of an estimate can be harder than making the estimate itself, which is usually hard enough. A proper error estimate for, say, a heat flux requires knowledge of the spatial and temporal variances of both the mass flux and the temperature field, and all the correlations between them. Obtaining all of this information is often impractical if not impossible. Nonetheless, some of the required information-such as the standard deviations of the transport and temperatures from their means - is often at hand. Use of this information can restrict the guesswork of uncertainty estimates to a few unknown but confined parameters, whose influence can be quantitatively evaluated. This provides a compromise between an unproducible, rigorous error bar on one hand, and an unconstrained, offhand guess - or worse, complete silence - on the other.

Even rough uncertainties of flux estimates are desirable for several reasons. They quantitatively assess the quality of the flux estimates and budgets. Uncertainties of boundary conditions are required by Arctic inverse models (e.g., Chapter 2). They are also the basis of statistical tests of hypotheses, which allow the simultaneous consideration of apparently contradictory data. Two examples are central to this chapter.

First, uncertainties allow different estimates to be combined into a better estimate. For any given Arctic boundary flux, such as the Bering Strait inflow, runoff, or the surface heat flux, several differing estimates exist (see below; also Rudels, 1987, Table 5). Including a flux in a budget requires one to guess which estimate is "best" and ignore the others. But with error bars available, one can hypothesize that the estimates all measure the same true transport, and that their differences are due to measurement errors. These errors are of course unknown, 
but their likely magnitudes are given by the uncertainties. This hypothesis can be statistically tested: if the differences among estimates are commensurate with the uncertainties, the estimates are consistent. In this case, it can be shown that the most likely value for the true flux is given by a weighted average of the previous estimates. This aggregate estimate will be consistent with the previous estimates, and its uncertainty will be smaller than any of the previous uncertainties.

Second, uncertainties allow flux estimates to be combined with other information. For example, previous budgets have quite reasonably insisted on mass conservation. But the terms of the mass budget contain errors, so the budget will not in general sum to zero. Further, a sum of exactly zero may not be a reasonable expectation: using volume as a proxy for mass, for example, creates an error of a few percent in the mass budget. ${ }^{1}$ In the past, such errors have been ignored. One boundary flux has been left unknown and then set to produce a zero total. With error bars known, one can instead adjust each flux estimate in proportion to its uncertainty, in order to bring the total within a specified tolerance of zero. The size of the tolerance reflects the exactness of the conservation statement. If the necessary adjustments are commensurate with the uncertainties in the data, then the data are consistent with the conservation statement. Otherwise either the data, the appropriateness of the conservation statement, or both must be questioned.

Based on published flux estimates and their authors' ad hoc uncertainty estimates, along with some ad hoc assumptions of my own, I will make rough (but quantitative) estimates of the Arctic boundary fluxes and their uncertainties. I will then use these to construct mass, heat, and salt budgets for the Arctic, and

\footnotetext{
${ }^{1}$ In general, there are other types of errors as well due to time-variations in the fluxes or to lack of completeness or synopticity in the data. For example, a mass budget for the Arctic could be in error if a temporary change in sea-level is not taken into account, or if a short-term surge in an inflow is captured by measurements while a short-term surge in outflow is missed. Another possibility is aliasing: if a variable flux is, by chance, sampled only when it is high, an overestimate results. For the steady model, flux estimates are presumed to be estimates of long-term average fluxes, and all such errors are presumed to be accounted for in the data uncertainties. This deliberately optimistic assumption is discussed below.
} 
to estimate the uncertainties of those budgets. The quantitative format of the uncertainty estimates will be used to determine which aspects of the budgets are heavily influenced by my assumptions.

\subsection{Notation and Terms}

For the purposes of this project the Arctic Ocean includes the Canadian and Eurasian Basins and their surrounding shelves, including the Barents Sea. The various exchanges across the boundaries of this region are divided into ten horizontal transports and five surface heat flux terms. The horizontal transports are

1. the Bering Strait inflow,

2. outflow through the Arctic Archipelago,

3. the inflow to the Barents Sea from the Norwegian Sea,

4. the freshwater input (runoff plus precipitation less evaporation),

and six exchanges through Fram Strait. The West Spitzbergen Current (WSC) flows northward through Fram Strait and is divided into

5. WSC Atlantic Water (to $1000 \mathrm{~m}$ ) and

6. WSC Deep Water (below $1000 \mathrm{~m}$ ).

The East Greenland Current (EGC) carries water southward, and is divided into

7. EGC ice,

8. EGC Polar Water (to approximately $250 \mathrm{~m}$ ),

9. EGC Atlantic Water (from 250 to $1000 \mathrm{~m}$ ), and

10. EGC Deep Water (below $1000 \mathrm{~m}$ ). 
The surface heat flux terms include

1. the flux through the surface of the deep basins,

as well as four shelf sea components chosen according to which estimates are available:

2. the flux from the permanently ice-free area of the southwestern Barents Sea,

3. the heat fluxes from winter polynyas surrounding the Canadian Basin and

4. from winter polynyas surrounding the Eurasian Basin, and

5. the remaining net flux from the shelves.

Each of the horizontal transports is presumed to have multi-year mean volume, potential temperature, and salinity anomaly fluxes, whose true (and unknown) values are denoted $\mathcal{Q}, \mathcal{H}$, and $\mathcal{L}$ respectively. From the quantities $\mathcal{Q}, \mathcal{H}$ and $\mathcal{L}$, two further quantities are defined: the transport potential temperature $\mathcal{T} \equiv \mathcal{H} / \mathcal{Q}$, and the transport salinity anomaly $\mathcal{S} \equiv \mathcal{L} / \mathcal{Q}$. A negative flux represents a flux out of the Arctic. Volume fluxes and budgets, expressed in Sverdrups, are used instead of mass; errors introduced by the implicit assumption of constant density (taken to be $1000 \mathrm{~kg} \mathrm{~m}^{-3}$ ) are negligible compared to the uncertainties in the volume flux estimates. Similarly, potential temperature fluxes are used instead of heat fluxes, and are expressed in $\mathrm{Sv}^{\circ} \mathrm{C}$. A difference of $1 \mathrm{~Sv}^{\circ} \mathrm{C}$ between two potential temperature fluxes is equivalent to a difference in heat fluxes of $4 \times 10^{12} \mathrm{~W}$. (This is obtained by multiplying $1 \mathrm{~Sv}^{\circ} \mathrm{C}$ by the product of the density and the heat capacity of sea water, taken here as a constant $4 \times 10^{6} \mathrm{~J} \mathrm{~m}^{-3} \mathrm{~K}^{-1}$.) Ignoring the small variations in the Arctic of the density-heat capacity product introduces errors which, again, are small compared to the uncertainties in the heat fluxes. Salinity anomaly fluxes are expressed in Sv·psu, where "psu" indicates that a volume flux has been multiplied by a dimensionless salinity anomaly, measured relative to an 
Arctic median of 34.4. A difference of $1 \mathrm{~Sv}$.psu between two salinity anomaly fluxes is very nearly equivalent to a difference of $10^{6} \mathrm{~kg} \mathrm{~s}^{-1}$ of salt.

Previously published estimates of these five quantities are denoted in lower case, $q_{i}, h_{i}, l_{i}, t_{i}, s_{i}$, where the index $i$ runs over the number of published estimates for a given flux. Aggregate estimates, formed from the various published estimates, are denoted in upper case, $Q, H, L, T, S$. Errors in the estimates are indicated by primes, so $q^{\prime} \equiv q-\mathcal{Q}, L^{\prime} \equiv L-\mathcal{L}$, etc. The variance of an estimate is defined as

$\sigma_{Q}^{2} \equiv\left\langle Q^{\prime 2}\right\rangle, \sigma_{s}^{2} \equiv\left\langle s^{2}\right\rangle$, etc., where $\langle\cdot\rangle$ indicates the expected or average value of a random quantity. The standard error, e.g., $\sigma_{Q}$, is the positive square root of the variance. (Note in particular that $\sigma_{t}$ represents the standard error of a transport potential temperature prior estimate, and not the potential density.) Values for an estimate and its standard error are sometimes written together as, e.g., $q \pm \sigma_{q}$. The covariance between the errors of two estimates, for example $Q$ and $H$, is defined as $\left\langle Q^{\prime} H^{\prime}\right\rangle$, from which their correlation coefficient is defined as $\rho_{Q H} \equiv\left\langle Q^{\prime} H^{\prime}\right\rangle / \sigma_{Q} \sigma_{H}$. The value of the correlation coefficient is necessarily between -1 and 1 .

\subsection{Previous Estimates}

The first step in generating the aggregate estimates is to standardize the available published estimates. For the purposes of this paper it would be ideal if each report presented estimates $q, h, l$, along with their error variances and covariances. What is usually presented is a subset of this information, or sometimes ancillary information which can be used to estimate these quantities. No paper (excepting Hanzlick, 1983) speculates on $\sigma_{h}$ or $\sigma_{l}$. Based on what is available, I've found it best to glean estimates from each paper of transports $(q)$, and where given, the transport temperatures $(t)$, transport salinity anomalies $(s)$ and the authors' assessments of transport uncertainties $\left(\sigma_{q}\right)$, then to proceed as well as possible in the absence of more direct information. 
When an estimate appears in more than one paper, I use (and cite) it only once. Summary papers such as Aagaard and Greisman (1975) are cited, but the many prior studies on which they are based are not, except when the prior studies provide additional relevant information. I have assumed that the cited authors' uncertainty estimates are correct, and accurately reflect the possibility of aliasing or other undersampling errors. The magnitude of this assumption should not be understated. At least three estimates are based on mooring data from the Fram Strait, an area known for variable and spatially concentrated jets. The Bering Strait transport is "known" to within $15 \%$, based largely on four years of data from three moorings. Long-term average transports are inferred from data collected in a month. A synoptic picture is assumed from data taken sporadically over decades, at different points and in different seasons. The uncertainties presented here are certainly underestimates, optimistically adopted as lower bounds. If one tried instead to set upper bounds on the uncertainty, its not clear where one would stop.

The specific treatment of each contributing paper is now summarized. The resulting estimates are presented in Table 3.1. For ease of reading, full salinities are discussed instead of the anomalies (differences from 34.4) used in calculations. The anomalies are listed in Table 3.1.

\subsubsection{Oceanic fluxes}

Aagaard and Carmack (1989) presented a comprehensive freshwater budget for the Arctic based on previous studies. Adding their runoff and precipitation less evaporation figures gives $q=0.13 \mathrm{~Sv}$ for the freshwater input. I arbitrarily assign $t=5 \pm 1{ }^{\circ} \mathrm{C}$ to freshwater; the flux is low enough that the heat budget is insensitive to this choice. Their Arctic Archipelago $q$ of $-1.7 \mathrm{~Sv}$ is from Fissel et al. (1988); the estimate of $\sigma_{q}=0.4 \mathrm{~Sv}$ is my own, based on the limited uncertainty information given in Fissel et al. 
Table 3.1: Estimates of transport $(q)$, transport standard error $\left(\sigma_{q}\right)$, transport potential temperature $(t)$, and transport salinity anomaly $(s)$ taken from or based on published reports. Blanks indicate that no estimate or basis for an estimate was given. See text for details.

\begin{tabular}{|c|c|c|c|c|}
\hline & $\begin{array}{c}q \\
(\mathrm{~Sv})\end{array}$ & $\begin{array}{c}\sigma_{q} \\
(\mathrm{~Sv})\end{array}$ & $\begin{array}{c}t \\
\left({ }^{\circ} \mathrm{C}\right)\end{array}$ & $\begin{array}{c}s \\
\text { (rel. to } 34.4 \text { ) }\end{array}$ \\
\hline \multicolumn{5}{|l|}{ Arctic Archipelago } \\
\hline Aagaard \& Greisman (1975) & -2.10 & 0.70 & -0.70 & -0.20 \\
\hline Rudels (1987) & -1.00 & 0.30 & -0.85 & -1.08 \\
\hline Aagaard \& Carmack (1989) & -1.70 & 0.40 & & \\
\hline Addison (1987) & -1.70 & & & \\
\hline \multicolumn{5}{|l|}{ Barents Sea } \\
\hline Mauritzen $(1996 a, b)$ & 1.60 & 0.30 & 4.00 & 0.65 \\
\hline Rudels (1987) & 1.20 & & 5.83 & 0.52 \\
\hline Blindheim (1989) & 1.90 & & 8.11 & 0.62 \\
\hline \multicolumn{5}{|l|}{ Bering Strait } \\
\hline Rudels (1987) & 0.80 & & -0.75 & -1.80 \\
\hline Roach et al. (1995) & 0.83 & 0.12 & -0.18 & -2.02 \\
\hline \multicolumn{5}{|l|}{ Freshwater } \\
\hline Rudels (1987) & 0.12 & & & \\
\hline Aagaard \& Carmack (1989) & 0.13 & & & \\
\hline \\
\hline Vinje et al. (1997) & -0.07 & 0.02 & & \\
\hline Hibler (1979) & -0.09 & & & \\
\hline Thomas et al.(1996) & -0.05 & & & \\
\hline Häkkinen (1993) & -0.06 & & & \\
\hline \multicolumn{5}{|l|}{ EGC Polar Water } \\
\hline Mauritzen $(1996 a, b)$ & -1.48 & 0.34 & -1.00 & -0.10 \\
\hline Rudels (1987) & -0.93 & & -1.42 & -0.21 \\
\hline Foldvik et al. (1988) & -1.00 & & -1.49 & -0.70 \\
\hline \multicolumn{5}{|l|}{ EGC Atlantic Water } \\
\hline Mauritzen $(1996 a, b)$ & -1.37 & 1.14 & 0.56 & 0.45 \\
\hline Rudels (1987) & -1.66 & & 1.31 & 0.52 \\
\hline Foldvik et al. (1988) & -2.00 & & 1.29 & 0.50 \\
\hline \multicolumn{5}{|l|}{ EGC Deep Water } \\
\hline Bönisch and Schlosser (1995) & -0.88 & 0.22 & -0.87 & 0.53 \\
\hline Rudels (1987) & -1.50 & & -0.56 & 0.50 \\
\hline Mauritzen $(1996 a, b)$ & -0.72 & 2.10 & & \\
\hline \multicolumn{5}{|l|}{ WSC Atlantic Water } \\
\hline Mauritzen $(1996 a, b)$ & 3.50 & 1.20 & 1.94 & 0.60 \\
\hline Aagaard \& Greisman (1975) & 7.10 & & 2.40 & 0.66 \\
\hline Hanzlick (1983) & 5.60 & 4.50 & 2.10 & \\
\hline Rudels (1987) & 1.90 & & 2.97 & 0.58 \\
\hline \multicolumn{5}{|l|}{ WSC Deep Water } \\
\hline Bönisch and Schlosser (1995) & 0.58 & 0.15 & -1.02 & 0.51 \\
\hline Mauritzen (1996a,b) & -0.48 & 1.30 & -1.00 & 0.51 \\
\hline Rudels (1987) & 1.10 & & -0.95 & 0.50 \\
\hline
\end{tabular}


Aagaard and Greisman (1975) gave full budgets of the Arctic oceanic flows based on studies to that date. Their WSC Atlantic Water transport of $q=7.1 \mathrm{~Sv}$ was based on current measurements and some limited hydrography. Their heat fluxes were given in $10^{9} \mathrm{kcal} \mathrm{s}^{-1}$, which I convert to a potential temperature transport of $h=17.06 \mathrm{~Sv}^{\circ} \mathrm{C}$ taking into account that their heat fluxes were relative to $-0.1^{\circ} \mathrm{C}$, and using constant density times heat capacity (Section 3.2). Dividing $h$ by $q$ gives $t=2.4{ }^{\circ} \mathrm{C}$. Their salt transport figures are nearly numerically equivalent to my salinity transport units, so I divide their $l=248.9 \mathrm{~Sv} \cdot$ psu by $q$ to get a transport salinity of 35.06. Their Arctic Archipelago figures were based on Muench (1971), which included summer measurements only, and are converted in the same way. Muench himself (1971) ascribed the uncertainty of $\sigma_{q}=0.7 \mathrm{~Sv}$ to the measurement.

Addison (1987) used hydrographic data in Nares Strait, Jones Sound and Lancaster Sound to estimate the geostrophic outflow of $q=1.7 \mathrm{~Sv}$ through the Arctic Archipelago. Based on this paper and on Fissel et al. (1988), the total flow through Jones and Lancaster Sounds appears to be approximately 1-5 times as large as the flow through Nares Strait.

Blindheim (1989) reported on current meter measurements of the fluxes between the Barents and Norwegian seas. Using Aagaard and Carmack's (1989) interpretation of his results gives $q=1.9 \mathrm{~Sv}$, and a transport salinity of 35.02. My own estimate of the heat flux from Blindheim's velocity and temperature sections produces $t=8.1^{\circ} \mathrm{C}$.

Bönisch and Schlosser (1995) estimated the deep fluxes through the Fram Strait by using hydrography and tracer data in an inverse box model. They cited their error $q / \sigma_{q}$ as $25-35 \%$; I take it as $25 \%$ in keeping with the "best case" philosophy described above.

Foldvik et al. (1988) measured the East Greenland Current Polar Water and Atlantic Water with current meter moorings. Based on autumn hydrography 
from Paquette et al. (1985), Aagaard and Carmack (1989) assigned to these flows transport salinities of 33.7 for the Polar Water and 34.9 for the Atlantic water.

Hanzlick (1983) used current meter moorings in the West Spitzbergen Current to estimate the flux of Atlantic Water to be $q=5.6 \mathrm{~Sv}$, with a transport temperature of $2{ }^{\circ} \mathrm{C}$. Since his heat flux was relative to $-0.1{ }^{\circ} \mathrm{C}$, I take $t=2.1^{\circ} \mathrm{C}$. Hanzlick said his rms errors could be "as large as the fluxes themselves", so I take $\sigma_{q}$ as $80 \%$ of $q$.

Mauritzen (1996a,b) compiled an 11-year database of hydrography from which she assigned $t$ and $s$, and used them in an inverse box model to estimate $q$ and $\sigma_{q} . I$ add her equations 7 and 14 to obtain estimates for the West Spitzbergen Current Atlantic Water, and add equations 30 and 31 to obtain estimates for the East Greenland Current Atlantic Water. Equations 20, 29, and 36 respectively give estimates for the WSC Deep Water, EGC Polar Water and EGC Deep Water (no $t$ or $s$ given). In addition to these uncertainties there is an unknown nullspace contribution which I have ignored. ${ }^{2}$

Roach et al. (1995) provided four years of current meter measurements of the flux through Bering Strait, resulting in an estimate of $q=0.83$ which they stated is good to $15 \%$. They did not estimate transport salinities or temperatures, but did give monthly figures of salinity, temperature, and transport from which I estimate $t=-0.18{ }^{\circ} \mathrm{C}$ and transport salinity 32.38 .

Rudels (1987) used hydrography of the Fram Strait to get the geostrophic velocity shear. Then, with previous estimates of all the other Arctic fluxes, he set the absolute velocities in Fram Strait to close the mass balance. I convert from his heat flux units assuming constant density times heat capacity as above, and divide the resulting temperature flux by volume flux to get transport temperature. In Fram Strait, Rudels's "Modified Atlantic Water" was comprised of two parts:

\footnotetext{
${ }^{2}$ For a definition of the solution nullspace see the footnote on page 35 . In Mauritzen's model, the nullspace spans all circulation patterns whose net effect in the constraining equations is zero.
} 
type IIa, which I add to the EGC Polar Water, and type IV, which I include in WSC Atlantic water. Salinity fluxes were not discussed by Rudels, so for lack of an alternative I hand pick transport salinities from the transport temperature and Rudels's temperature-salinity correlations. For the Arctic Archipelago figures he cited Rudels (1986), in which he stated that the exiting surface flow is unlikely to be outside of -0.5 to $-1.2 \mathrm{~Sv}$. Interpreting this as $-0.7 \pm 0.2 \mathrm{~Sv}$ and giving the same uncertainty to his guess of $-0.3 \mathrm{~Sv}$ for the deep flow gives the total estimate of $q=-1.0 \pm 0.3$

\subsubsection{Ice fluxes}

By far the largest flux of ice across the Arctic boundary is that out of the Fram Strait through the East Greenland Current. All other ice fluxes are negligible (Rudels, 1987). To enable comparison of the effects of ice and liquid transports on the mass budgets, ice volume transports have been multiplied by the density ratio of ice to water (0.9).

Ice export is a large term in the heat budget owing to the very low internal energy of ice relative to liquid water: liquid water loses a great deal of latent heat as it freezes (see Maykut, 1985). To reflect this, the effective transport potential temperature for ice is estimated by dividing the internal energy of sea ice (relative to sea water at $\left.0^{\circ} \mathrm{C}\right)$ by the liquid density and heat capacity $\left(4 \times 10^{6} \mathrm{~J} \mathrm{~m}^{-3} \mathrm{~K}^{-1}\right)$, yielding $t=-83.72 \pm 1.00^{\circ} \mathrm{C}$.

The salinity of Arctic sea ice varies depending on its age and on details of its formation (see Maykut, 1985). The ice exiting through Fram Strait is assumed here to be older ice with a transport salinity of $3 \pm 1$.

The equivalent liquid volume flux through Fram Strait is estimated from the following previous estimates:

Häkkinen (1993) used a wind-driven numerical ice and ocean model to es- 
timate a mean Fram Strait ice volume export of $2000 \mathrm{~km}^{3}$ per year, implying $q=-0.06 \mathrm{~Sv}$.

Hibler (1979), also using a numerical ice model, estimated a mean Fram Strait ice volume export of $3220 \mathrm{~km}^{3}$ per year, or $q=-0.09 \mathrm{~Sv}$.

Thomas et al. (1996) assimilated satellite ice concentration and buoy data into a thermodynamic ice growth model to estimate net ice growth and advection in the Arctic from 1979-1985. Their results give $q=-0.05 \mathrm{~Sv}$ through the Fram Strait.

Vinje et al. (1997) used upward-looking sonar to measure ice thickness distribution in Fram Strait. This was combined with satellite data to obtain ice volume flux estimates from 1990-1994, whose average $q$ is $-0.07 \pm 0.02 \mathrm{~Sv}$. This study superseded the widely-cited paper of Vinje and Finnekåsa (1986) which used a similar methodology but an ice-thickness distribution now known to overestimate the annual average. The uncertainty estimate is my own, based on Vinje et al.'s discussion of sources of error.

\subsubsection{Surface heat fluxes}

The surface heat fluxes are the least well known components of the Arctic budgets, and little guidance is available for their estimation. SCOR-WG 58 (1979) presents a summary to that date. More recent work (Maykut, 1982; Maykut and McPhee, 1995 ) indicates that the picture is greatly complicated by the presence of leads and other inhomogeneities of the ice cover-not to mention that to this point, most studies have examined only the vertical dimension. It is difficult to establish even the sign of the heat flux with rigor. For example, Arctic surface air temperatures are around $-30^{\circ} \mathrm{C}$ in January, and near $0^{\circ} \mathrm{C}$ in July (SCOR-WG 58, 1979). An

annual average temperature difference of $15^{\circ} \mathrm{C}$ across a typical ice cover $3 \mathrm{~m}$ thick with a thermal conductivity of $2 \mathrm{~W} \mathrm{~K}^{-1} \mathrm{~m}^{-1}$ (Maykut, 1985, p. 46) gives a sensible 
heat loss to the atmosphere of approximately $10 \mathrm{~W} \mathrm{~m}^{-2}$. Now, this figure should be reduced owing to the excellent insulation properties of snow. But then it should be augmented again, to account for increased heat loss when the wind is blowing. It should then be adjusted further due to leads in the ice pack, which include only $1 \%$ of the area, but which are open to radiative heat loss and insolation, and therefore may dominate the heat budget of the central Arctic (SCOR-WG 58, 1979). The net sign of the contribution of leads is not certain. How to sum so many unknowns?

The prevailing opinion is that in the permanently ice-covered central Arctic, the net surface heat flux is not likely to be very large. This conclusion is based principally on ice models which indicate that it would be hard to maintain the observed ice thickness distribution if the net annual surface heat flux were very different from zero (G. Maykut, personal communication, 1996). Here I adopt a heat flux estimate of $0 \pm 5 \mathrm{~W} \mathrm{~m}^{-2}$. Note that I deliberately make the uncertainty small, to represent my belief that the total heat flux is small. That uncertainty does not represent the accuracy of direct measurements: heat flux observations are subject to errors at least an order of magnitude greater than $5 \mathrm{~W} \mathrm{~m}^{-2}$. Multiplying $0 \pm 5 \mathrm{~W} \mathrm{~m}^{-2}$ by the area of the central Arctic $\left(5.95 \times 10^{12} \mathrm{~m}^{2}\right)$ and dividing by the density and heat capacity of liquid sea water $\left(4 \times 10^{6} \mathrm{~J} \mathrm{~m}^{-3} \mathrm{~K}^{-1}\right)$ gives an equivalent "temperature flux" of $h=0 \pm 7.4 \mathrm{~Sv}^{\circ} \mathrm{C}$.

The assumption of zero heat flux as a central value may seem inconsistent with the idea that heat from lower latitudes is lost to the atmosphere at the poles. In fact, there is no inconsistency, as most of the heat loss occurs at the margins of the Arctic (Aagaard and Carmack, 1994). In the end, however, the central value is prescribed to be zero because that is a simple choice for an unknown parameter whose sign is unknown and whose size is small. One may thus legitimately wonder what the effect on the model results would be if I had chosen $-5 \mathrm{~W} \mathrm{~m}^{-2}$ or $5 \mathrm{~W} \mathrm{~m}^{-2}$ instead of zero. To put the reader's mind at ease, this issue is addressed in two ways. First, based on my choice of $0 \pm 5 \mathrm{~W} \mathrm{~m}^{-2}$, the Monte Carlo simulations vary the 
basin surface heat flux in a normal distribution over that range; thus the Monte Carlo trials explore solutions with basin surface heat fluxes from approximately -10 to $10 \mathrm{~W} \mathrm{~m}^{-2}$. Second, a series of calculations is performed in which the basin surface heat flux is varied systematically through this range; those calculations are presented in Section 4.4.7.

The heat flux over the shelves is as unknown as that over the basin, but components of it have been estimated. Martin and Cavalieri (1989) and Cavalieri and Martin (1994) used satellite and weather data to estimate the heat lost from winter coastal polynyas after the water column had reached the freezing point, that is, the heat loss which directly caused ice formation. Their figures depended on the date at which the freezing point was reached, which is unknown. I average the estimates based on their "early" and "late" dates, then average over all the years of the study. Polynyas located in the Bering Sea are outside the control area of this study and are excluded. The resulting winter heat fluxes are averaged over an entire year to give final figures of $-5.4 \times 10^{12} \mathrm{~W}$ from Canadian Basin shelf polynyas, and $-2.2 \times 10^{12} \mathrm{~W}$ from Eurasian Basin shelf polynyas. Converting to equivalent temperature transports and using Cavalieri and Martin's uncertainty estimate of $35 \%$ gives $h=-1.3 \pm 0.5 \mathrm{~Sv}^{\circ} \mathrm{C}$ for the Canadian Basin shelves and $h=-0.6 \pm 0.2 \mathrm{~Sv}^{\circ} \mathrm{C}$ for the Eurasian Basin shelves.

The surface heat flux maps of Bunker and Worthington (1976) cover the permanently ice-free area of the southwestern Barents Sea. An estimate from their figures gives a heat flux of $-70 \mathrm{kcal} \mathrm{cm}^{-2} \mathrm{yr}^{-1}$, which with an estimated ice-free area of $6 \times 10^{11} \mathrm{~m}^{2}$ (Rudels, 1987) implies an equivalent temperature transport of $h=-14 \mathrm{~Sv}^{\circ} \mathrm{C}$. I arbitrarily assign an error of $45 \%$, or $\sigma_{h}=6 \mathrm{~Sv}^{\circ} \mathrm{C}$. This choice implies that the heat flux is known well enough to determine the sign, but not much better than that.

These individual components of the shelf heat flux are interesting, but are only pieces of the net total annual heat flux over the shelves. I lump the remainder of 
the heat flux into one unknown term. The net remaining flux is probably the sum of a large loss in the fall and a large gain in the spring, making its size and sign uncertain. For lack of information, I arbitrarily double the uncertainty used for the ice-covered central Arctic and estimate the net of all remaining shelf surface heat fluxes to be $0 \pm 10 \mathrm{~W} \mathrm{~m}^{-2}$. Taking the shelf area as $3.35 \times 10^{12} \mathrm{~m}^{2}$ gives an equivalent temperature flux of $0 \pm 8.4 \mathrm{~Sv}^{\circ} \mathrm{C}$.

\subsection{Aggregate Estimates}

The standardized prior estimates (Table 3.1) are next combined into aggregate estimates $Q, H$, and $L$ for each flow (Bering Strait, Arctic Archipelago, etc.). Variances and co-variances of the aggregate estimates are also generated.

\subsubsection{Volume fluxes}

The aggregate estimate $Q \pm \sigma_{Q}$ is a weighted average of the $n$ prior estimates

$$
q_{1} \pm \sigma_{q_{1}}, q_{2} \pm \sigma_{q_{2}}, \ldots, q_{i} \pm \sigma_{q_{i}}, \ldots, q_{n} \pm \sigma_{q_{n}} .
$$

The weights for the average are chosen to minimize the variance $\sigma_{Q}$, as follows. ${ }^{3}$

The first step in determining $Q$ is to assign uncertainties to the $q$ 's where authors did not provide them. Previous estimates without uncertainties receive a default uncertainty equal to $50 \%$ of the mean of all transport estimates for the flow. The effect of this choice on the aggregate estimates is discussed below.

Each $q$ is assumed to be a realization of a random variable, normally distributed with mean $\mathcal{Q}$ and variance $\sigma_{q_{i}}^{2}$. The $q$ 's are further assumed to be mutually independent. Mandating that $Q$ be a linear combination of the $q$ 's gives

$$
Q=a_{1} q_{1}+a_{2} q_{2}+\ldots+a_{n} q_{n}
$$

\footnotetext{
${ }^{3} \mathrm{~A}$ "maximum likelihood" approach can also be used to select the estimator $Q$. In that case, $Q$ is chosen (through the weights) to maximize the probability of the $q$ 's having their observed values. For the Gaussian variables in question here, the two methods are equivalent.
} 
with the $a$ 's to be determined. Standard results for linear combinations of Gaussian variables (e.g., Priestley, 1981) show that $Q$ is itself a realization of a Gaussian variable. It can also be shown that $Q$ is an unbiased estimator of $\mathcal{Q}($ i.e., $\langle Q\rangle=\mathcal{Q}$ ) if $\sum_{i=1}^{n} a_{i}=1$. Subject to this requirement, the $a$ 's are chosen to minimize the variance $\sigma_{Q}^{2}$. These turn out to be

$$
a_{i}=b_{i} / \sum_{j=1}^{n} b_{j}, b_{i} \equiv \prod_{k \neq i} \sigma_{q_{k}}^{2}
$$

(See also Wunsch, 1996, p. 208). To aid interpretation the $a$ 's are written out for $n=3$ :

$$
\begin{aligned}
& a_{1}=\frac{\sigma_{q_{2}}^{2} \sigma_{q_{3}}^{2}}{\sigma_{q_{2}}^{2} \sigma_{q_{3}}^{2}+\sigma_{q_{1}}^{2} \sigma_{q_{3}}^{2}+\sigma_{q_{1}}^{2} \sigma_{q_{2}}^{2}}, \\
& a_{2}=\frac{\sigma_{q_{1}}^{2} \sigma_{q_{3}}^{2}}{\sigma_{q_{2}}^{2} \sigma_{q_{3}}^{2}+\sigma_{q_{1}}^{2} \sigma_{q_{3}}^{2}+\sigma_{q_{1}}^{2} \sigma_{q_{2}}^{2}}, \\
& a_{3}=\frac{\sigma_{q_{1}}^{2} \sigma_{q_{2}}^{2}}{\sigma_{q_{2}}^{2} \sigma_{q_{3}}^{2}+\sigma_{q_{1}}^{2} \sigma_{q_{3}}^{2}+\sigma_{q_{1}}^{2} \sigma_{q_{2}}^{2}} .
\end{aligned}
$$

The variance of $Q$ is $\sigma_{Q}^{2}=\sum_{i=1}^{n} a_{i}^{2} \sigma_{q_{i}}^{2}$, and it can be shown that $\sigma_{Q}^{2}$ is necessarily less than or equal to any of the prior variances $\sigma_{q_{i}}^{2}$. It is no surprise that $Q$ is a better estimate of $\mathcal{Q}$ than any of the prior estimates since $Q$ contains more information than does any individual $q$. Another advantage of the aggregate estimates is that they efficiently represent the prior estimates: the weighted mean of a further estimate $q_{\text {new }} \pm \sigma_{q_{\text {new }}}$ with $Q \pm \sigma_{Q}$ is the same as the weighted mean of $q_{\text {new }} \pm \sigma_{q \text { new }}$ with all the previous $q$ 's.

Aggregate estimates $Q \pm \sigma_{Q}$ for each of the Arctic fluxes are presented in Table 3.2. Plots comparing each $Q$ and $\sigma_{Q}$ to its component $q$ 's and $\sigma_{q}$ 's appear in Figure 3.1. Notably, none of the published estimates for a given flow contradict one another; they all overlap within error bars. Thus there is no contradiction of the prior assumption that each $q$ successfully reflects the same true value $\mathcal{Q}$.

The effect of the default uncertainty on the aggregate estimates $Q$ can be explored using (3.2). The value of $Q$ tends toward the estimates with the smallest 
Table 3.2: Aggregate Arctic flux estimates with standard errors and covariances, based on previously published estimates. Temperature and salinity anomaly fluxes, their variances, and covariances are calculated assuming $\rho_{Q T}=\rho_{Q S}=\rho_{S T}=0$. Totals assume errors between different flux estimates are uncorrelated.

Budget 1

\begin{tabular}{|c|c|c|c|c|c|c|c|c|c|}
\hline \multicolumn{10}{|c|}{ Aggregate Estimates } \\
\hline \multirow[b]{3}{*}{ Arctic Arch } & $Q$ & $\sigma_{Q}$ & $H$ & $\sigma_{H}$ & $L$ & $\sigma_{L}$ & $\left\langle Q^{\prime} H^{\prime}\right\rangle$ & $\left\langle Q^{\prime} L^{\prime}\right\rangle$ & $\left\langle H^{\prime} L^{\prime}\right\rangle$ \\
\hline & \multicolumn{2}{|c|}{$\mathrm{Sv}$} & \multicolumn{2}{|c|}{$\mathrm{Sv}^{\circ} \mathrm{C}$} & \multicolumn{2}{|c|}{$\mathrm{Sv}(\mathrm{psu})$} & $\mathrm{Sv}^{2 \circ} \mathrm{C}$ & $S v^{2}(p s u)$ & $\mathrm{Sv}^{20} \mathrm{C}(\mathrm{psu})$ \\
\hline & -1.37 & 0.22 & 1.06 & 0.22 & 0.87 & 0.86 & -0.04 & -0.03 & 0.02 \\
\hline Barents Sea & 1.59 & 0.26 & 9.50 & 3.63 & 0.95 & 0.22 & 0.42 & 0.04 & 0.25 \\
\hline Bering Strait & 0.83 & 0.12 & -0.38 & 0.34 & -1.58 & 0.26 & -0.01 & -0.03 & 0.01 \\
\hline Freshwater & 0.13 & 0.04 & 0.63 & 0.33 & -4.30 & 1.52 & 0.01 & -0.07 & -0.34 \\
\hline \multicolumn{10}{|l|}{ Fram Strait: } \\
\hline Ice & -0.07 & 0.01 & 5.72 & 1.17 & 2.14 & 0.44 & -0.02 & -0.01 & 0.51 \\
\hline EGC PW & -1.27 & 0.26 & 1.65 & 0.48 & 0.43 & 0.41 & -0.09 & -0.02 & 0.03 \\
\hline EGC AW & -1.73 & 0.53 & -1.82 & 0.92 & -0.85 & 0.31 & 0.29 & 0.14 & 0.14 \\
\hline EGC DW & -0.97 & 0.20 & 0.70 & 0.26 & -0.50 & 0.14 & -0.03 & 0.02 & -0.01 \\
\hline WSC AW & 3.94 & 0.94 & 9.26 & 2.84 & 2.41 & 0.70 & 2.07 & 0.54 & 1.27 \\
\hline WSC DW & 0.75 & 0.12 & -0.74 & 0.14 & 0.38 & 0.10 & -0.01 & 0.01 & -0.01 \\
\hline \multicolumn{10}{|l|}{ Surface Heat Flux: } \\
\hline Basin & & & 0.00 & 7.44 & & & & & \\
\hline Ice-free Barents & & & -13.94 & 6.27 & & & & & \\
\hline CB Polynyas & & & -1.34 & 0.47 & & & & & \\
\hline EB Polynyas & & & -0.56 & 0.20 & & & & & \\
\hline Shelf: Other & & & 0.00 & 8.37 & & & & & \\
\hline TOTAL (NET) & 1.82 & 1.19 & 9.72 & 13.75 & -0.04 & 2.04 & & & \\
\hline
\end{tabular}



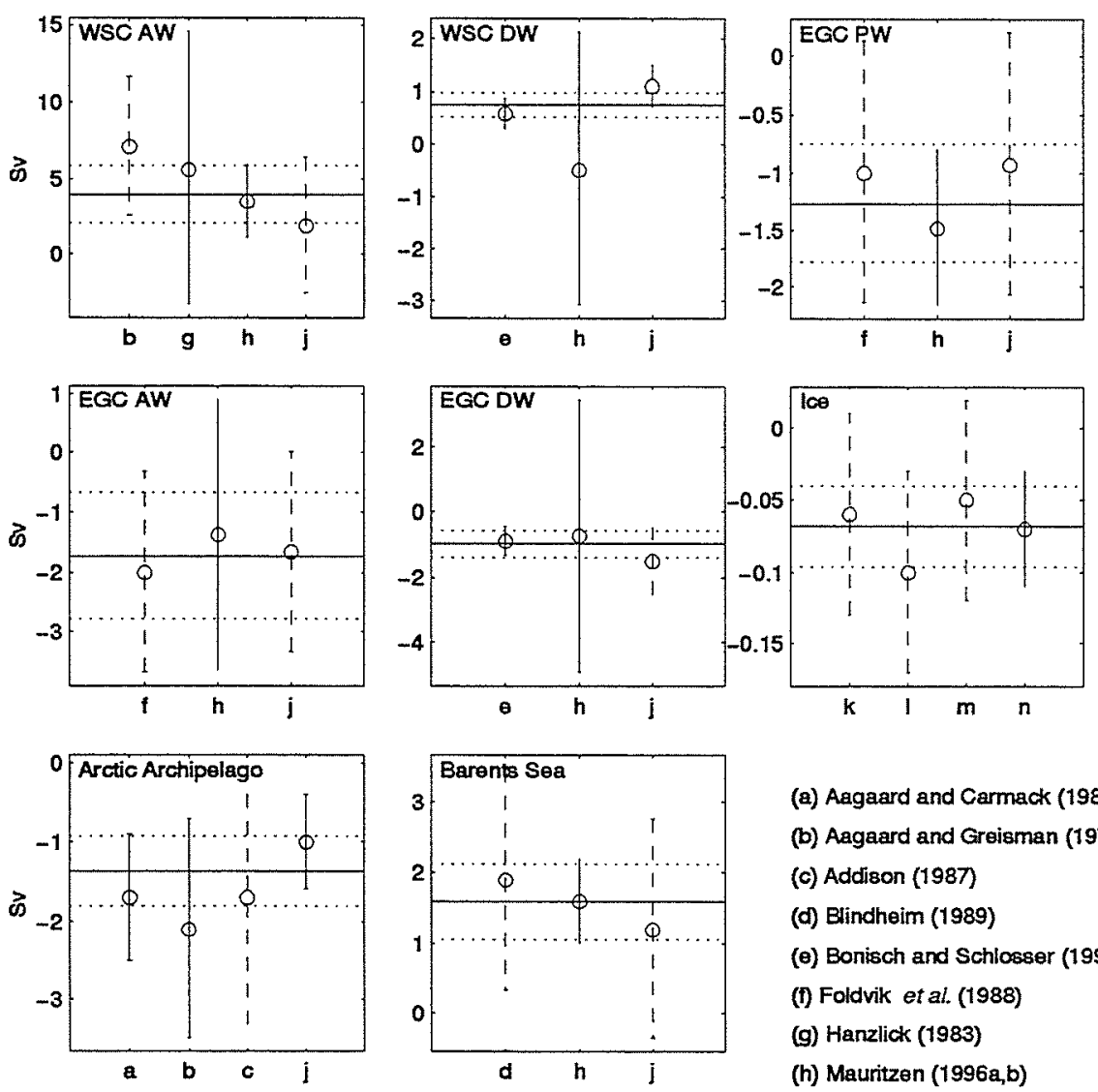

(a) Aagaard and Carmack (1989)

(b) Aagaard and Greisman (1975)

(c) Addison (1987)

(d) Blindheim (1989)

(e) Bonisch and Schlosser (1995)

(f) Foldvik et al. (1988)

(g) Hanzlick (1983)

(h) Mauritzen (1996a,b)
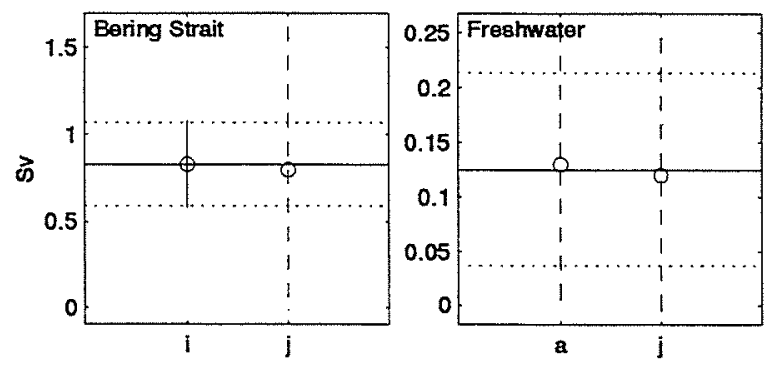

(i) Roach et al. (1995)

(1) Rudels (1987)

(k) Hakkinen (1993)

(I) Hibler (1979)

(m) Thomas et al. (1996)

(n) Vinje et al. (1997)

Figure 3.1: Previous and aggregate transport estimates for the 10 Arctic boundary flows. Previous estimates and two-standard-error error bars are marked with circles and vertical lines. Dashed error bars are defaults, used when no error estimate was provided with the transport estimate. Estimate sources are indicated by letter according to the key at lower right. Horizontal lines indicate aggregate estimates (solid) and their error bars (dotted). 
uncertainties. A small default will force $Q$ toward those estimates to which the default is applied, while a large default will reduce the influence of those estimates. Therefore the $Q$ 's will not be sensitive to the default $\sigma_{q}$ so long as the default is not made small. For the Arctic fluxes, the default uncertainty I've chosen is about the same size or slightly larger than the published uncertainty estimates (Figure 3.1).

The aggregate uncertainty $\sigma_{Q}$ is theoretically also affected by the choice of the default $\sigma_{q}$. However, in practice $\sigma_{Q}$ is determined principally by the smallest $\sigma_{q}$, which is rarely the default value (Figure 3.1); therefore $\sigma_{Q}$ for the Arctic fluxes is rather insensitive to the default $\sigma_{q}$. The exception is the freshwater flux, which has no prior uncertainty estimates and for which $\sigma_{Q}$ is determined entirely by the choice of the default.

\subsubsection{Potential temperature and salinity anomaly fluxes}

Having derived transport estimates $Q \pm \sigma_{Q}$ for each flow, it remains to estimate the heat fluxes $H \pm \sigma_{H}$ and salt fluxes $L \pm \sigma_{T}$, along with their covariances $\left\langle Q^{\prime} H^{\prime}\right\rangle$, $\left\langle H^{\prime} L^{\prime}\right\rangle$, etc. These will turn out to depend on the unknown correlations between transports, temperatures, and salinities, so the dependence of the estimates on those unknowns will also be explored.

Ideally, aggregate estimates of heat and salt fluxes would be generated in the same manner as those of volume fluxes. Unfortunately, uncertainties of the published heat and salt flux estimates are not available. Assuming they are proportional to the volume transport uncertainties ignores the sometimes large contribution of uncertainties in the transport temperature or salinity. These latter uncertainties are included by obtaining rough estimates of the transport temperatures and salinities, and of their uncertainties, as follows.

For each flow, prior estimates of the transport potential temperatures $t$ and transport salinity anomalies $s$ are obtained from the literature as described in 
Section 3.3. Since uncertainties for the $t$ 's and $s$ 's are not available, the aggregate estimates of $T$ and $S$ are taken to be straight averages: $T=\left(t_{1}+t_{2}+\ldots+t_{n}\right) / n$, and similarly for $S$.

The uncertainties of straight averages such as $T$ and $S$ are conventionally estimated by the standard deviation divided by $n^{1 / 2}$, but this is not valid for the very small sample sizes in this study. Instead, the initial estimates of $\sigma_{T}$ and $\sigma_{S}$ are taken to be the sample standard deviations, e.g., $\sigma_{T}^{2}=\sum_{i=1}^{n}\left(t_{i}-T\right)^{2} /(n-1)$. However, even this can be unreasonably small if the t's or $s$ 's are all very close together, resulting in a near-zero uncertainty based on only two or three estimates which happen to closely match. Therefore the final estimates of $\sigma_{T}$ and $\sigma_{S}$ are taken to be the larger of the sample standard deviation or a default minimum. In keeping with this study's optimistic attitude regarding uncertainties, the default minima are set to $0.1{ }^{\circ} \mathrm{C}$ for temperature and 0.1 for salinity. In practice, the defaults are used for the transport temperature of the WSC Deep Water, and for the transport salinity anomalies of the Barents Sea inflow and the WSC and EGC Atlantic and Deep Waters.

In each of the previous studies, the errors in the flux estimates $\left(q^{\prime}, t^{\prime}\right.$, and $s^{\prime}$ ) are certainly not independent: estimates of transport, transport temperature and transport salinity all depend on the estimate of the velocity field. An extreme case is geostrophic calculations, in which the estimates of the velocity, temperature and salinity fields are all functions of the same hydrographic measurements. The strengths of these error correlations are not estimated in the Arctic literature. Though one might hope, as I do below, that averaging together the results of several studies might soften these correlations, it must be acknowledged that the errors of the resulting aggregate estimates, $Q^{\prime}, T^{\prime}$, and $S^{\prime}$ are still correlated, and that their correlation coefficients $\rho_{Q T}, \rho_{Q S}$, and $\rho_{S T}$ are unknown. Writing subsequent results in terms of these unknown coefficients will allow investigation of their importance. 
Aggregate estimates of the potential temperature flux are derived as follows. By definition, $\mathcal{H} \equiv \mathcal{Q T} \equiv\left(Q+Q^{\prime}\right)\left(T+T^{\prime}\right)$. Let $H=\left\langle\left(Q+Q^{\prime}\right)\left(T+T^{\prime}\right)\right\rangle$, and note that $\left\langle Q^{\prime}\right\rangle=\left\langle T^{\prime}\right\rangle=0$. Also note that $Q$ and $T$ are calculated, not random. Then

$$
H=Q T+\left\langle Q^{\prime} T^{\prime}\right\rangle=Q T+\sigma_{Q} \sigma_{T} \rho_{Q T} .
$$

Similarly expanding $\sigma_{H}^{2}=\left\langle H^{\prime 2}\right\rangle=\left\langle(H-\mathcal{H})^{2}\right\rangle$ gives

$\sigma_{H}^{2}=T^{2} \sigma_{Q}^{2}+Q^{2} \sigma_{T}^{2}+2 Q T \sigma_{Q} \sigma_{T} \rho_{Q T}-\rho_{Q T}^{2} \sigma_{Q}^{2} \sigma_{T}^{2}+2 Q\left\langle Q^{\prime} T^{\prime 2}\right\rangle+2 T\left\langle T^{\prime} Q^{\prime 2}\right\rangle+\left\langle Q^{\prime 2} T^{\prime 2}\right\rangle$.

It will be helpful to express the last three terms of (3.4) in terms of known quantities and the single unknown $\rho_{Q T}$. The correlation of $Q^{\prime}$ and $T^{\prime}$ implies that

$$
T^{\prime}=\rho_{Q T} \frac{\sigma_{T}}{\sigma_{Q}} Q^{\prime}+n
$$

where $n$ is that part of $T^{\prime}$ independent of $Q^{\prime}$ (Wunsch, 1996, p. 104). Use of (3.5) yields

$$
\begin{aligned}
& \left\langle Q^{\prime} T^{\prime 2}\right\rangle=\left\langle n^{2} Q^{\prime}\right\rangle+2 \rho_{Q T} \frac{\sigma_{\mathcal{T}}}{\sigma_{Q}}\left\langle Q^{\prime 2} n\right\rangle+\rho_{Q T}^{2} \frac{\sigma_{T}^{2}}{\sigma_{Q}^{2}}\left\langle Q^{\prime 3}\right\rangle=0 \\
& \left\langle Q^{\prime 2} T^{\prime}\right\rangle=\left\langle Q^{\prime 2} n\right\rangle+\rho_{Q T} \frac{\sigma_{T}}{\sigma_{Q}}\left\langle Q^{\prime 3}\right\rangle=0 \\
& \left\langle Q^{\prime 2} T^{\prime 2}\right\rangle=\left\langle Q^{\prime 2} n^{2}\right\rangle+2 \rho_{Q T} \frac{\sigma_{T}}{\sigma_{Q}}\left\langle Q^{\prime 3} n\right\rangle+\rho_{Q T}^{2} \frac{\sigma_{T}^{2}}{\sigma_{Q}^{2}}\left\langle Q^{\prime 4}\right\rangle=3 \rho_{Q T}^{2} \sigma_{Q}^{2} \sigma_{T}^{2} .
\end{aligned}
$$

In deriving these results it is inferred from the definition of $n$ that any power of $Q^{\prime}$ is independent of any power of $n$. Also used are $\left\langle Q^{\prime 3}\right\rangle=0$, since $Q^{\prime}$ is assumed to be Gaussian and therefore symmetrically distributed around 0 , and $\left\langle Q^{\prime 4}\right\rangle=3 \sigma_{Q}^{4}$. Substituting the results (3.6) into (3.4) gives

$$
\sigma_{H}^{2}=T^{2} \sigma_{Q}^{2}+Q^{2} \sigma_{T}^{2}+2 Q T \sigma_{Q} \sigma_{T} \rho_{Q T}+2 \rho_{Q T}^{2} \sigma_{Q}^{2} \sigma_{T}^{2} .
$$

Using similar reasoning, the covariance simplifies to

$$
\left\langle Q^{\prime} H^{\prime}\right\rangle=T \sigma_{Q}^{2}+Q \sigma_{Q} \sigma_{T} \rho_{Q T}
$$


The correlation coefficient of $Q^{\prime}$ and $H^{\prime}$ can be expressed as

$$
\rho_{Q H}=\frac{\bar{Q} \rho_{Q T}+\bar{T}}{\left(\bar{Q}^{2}+\bar{T}^{2}+2 \bar{Q} \bar{T} \rho_{Q T}+2 \rho_{Q T}^{2}\right)^{1 / 2}}
$$

where $\bar{Q}=Q / \sigma_{Q}$ and $\bar{T}=T / \sigma_{T}$.

The estimations of the salinity anomaly flux $L$ and its associated quantities $\sigma_{L}^{2}$, $\left\langle Q^{\prime} L^{\prime}\right\rangle$ and $\rho_{Q L}$ are identical to those for $H$. The last covariance is that between temperature and salinity anomaly flux estimates, and is given by

$$
\left\langle H^{\prime} L^{\prime}\right\rangle=S T \sigma_{Q}^{2}+Q T \sigma_{Q} \sigma_{S} \rho_{Q S}+Q S \sigma_{Q} \sigma_{T} \rho_{Q T}+Q^{2} \sigma_{S} \sigma_{T} \rho_{S T}+2 \sigma_{Q}^{2} \sigma_{T} \sigma_{S} \rho_{Q T} \rho_{Q S}
$$

where results similar to (3.6) have been used. The correlation coefficient is given by

$$
\rho_{H I}=\frac{\bar{Q}^{2} \rho_{T S}+\bar{Q} \bar{S} \rho_{Q T}+\bar{Q} \bar{T} \rho_{Q S}+\bar{T} \bar{S}+2 \rho_{Q T} \rho_{Q S}}{\left[\left(\bar{Q}^{2}+\bar{T}^{2}+2 \rho_{Q T} \bar{Q} \bar{T}+2 \rho_{Q T}^{2}\right)\left(\bar{Q}^{2}+\bar{S}^{2}+2 \rho_{Q S} \bar{Q} \bar{S}+2 \rho_{Q S}^{2}\right)\right]^{1 / 2}}
$$

with $\bar{S}=S / \sigma_{S}$.

Aggregate estimates for each flow have been calculated using the formulas above, assuming $\rho_{Q T}=\rho_{Q S}=\rho_{S T}=0$ (Table 3.2). The assumption of zero correlations might be justified on the basis of simplicity, or one might argue that the correlations among the individual $q$ 's, $t$ 's, and $s$ 's are partly canceled in the average. In any case, the effect of this assumption on the estimates can be determined from the equations. It is evident from (3.3) that $\rho_{Q T}$ will only affect $H$ if $\sigma_{Q} \sigma_{T} / Q T$ is $O(1)$ or larger. In the aggregate estimates of this study, the absolute value of this ratio is highest for the freshwater input (0.14), Bering Strait $(-0.12)$ and East Greenland Current Atlantic Water $(-0.12)$. The magnitude of the ratio is less than 0.10 for all other flows. Thus the present estimates of temperature flux are not very sensitive to $\rho_{Q T}$. The dependence of $L$ on $\rho_{Q S}$ is exactly analogous, and the results are similar: the most sensitive estimates are those for EGC Polar 

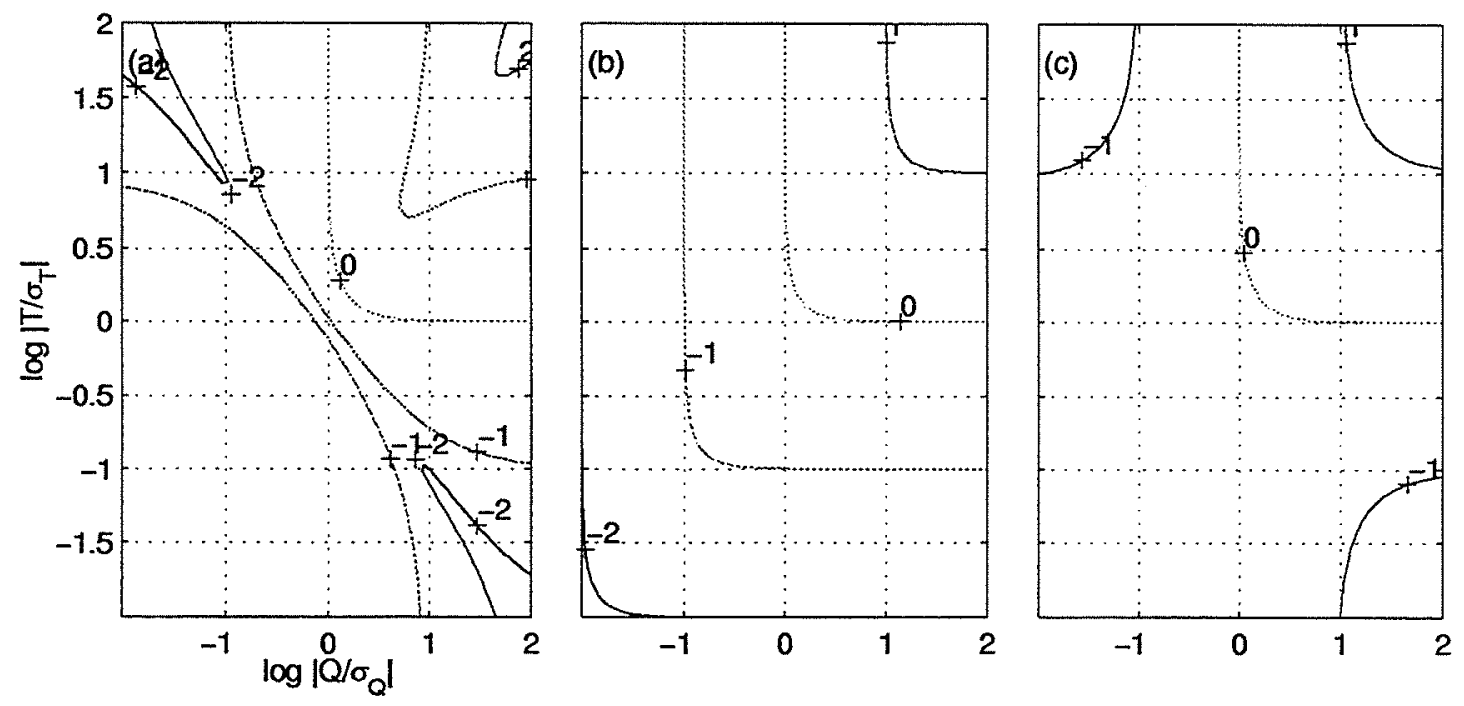

Figure 3.2: Contours of $\log \left|H / \sigma_{H}\right|$ vs. $\log \left|T / \sigma_{T}\right|$ and $\log \left|Q / \sigma_{Q}\right|$. (a) $\rho_{Q T}=-0.9$, $Q T>0$, or $\rho_{Q T}=0.9, Q T<0$. (b) $\rho_{Q T}=0$. (c) $\rho_{Q T}=0.9, Q T>0$, or $\rho_{Q T}=-0.9, Q T<0$. Contour values are -2 to 2 by 1 .

Water $\left(\sigma_{Q} \sigma_{S} / Q S=0.19\right)$ and the Arctic Archipelago $\left(\sigma_{Q} \sigma_{S} / Q S=0.16\right)$. For all other flows, the absolute value of $\sigma_{Q} \sigma_{S} / Q S$ is less than 0.07 .

The effect of $\rho_{Q T}$ on the statistical significance of $H$ can be seen by plotting the quantity $H / \sigma_{H}$, which from (3.3) and (3.7) can be shown to be

$$
\frac{H}{\sigma_{H}}=\frac{\bar{Q} \bar{T}+\rho_{Q T}}{\left(\bar{Q}^{2}+\bar{T}^{2}+2 \bar{Q} \bar{T} \rho_{Q T}+2 \rho_{Q T}^{2}\right)^{1 / 2}}
$$

Figure 3.2 shows contours of $H / \sigma_{H}$ as a function of $\bar{Q}$ and $\bar{T}$ for different values of $\rho_{Q T}$. If $\bar{Q}$ and $\bar{T}$ are both greater than 1 (low uncertainty), then $H / \sigma_{H}$ is also greater than one; $\rho_{Q T}$ only determines how much greater. If one of $\bar{Q}$ or $\bar{T}$ is greater than one, then the other, more uncertain estimate renders the temperature flux uncertain as well, regardless of $\rho_{Q T}$. If $\bar{Q}$ and $\bar{T}$ are both less than one (both uncertain), then $H / \sigma_{H}$ depends more strongly on $\rho_{Q T}$ : the size and sign of the temperature flux is largely determined by the strength of the correlation between the large errors in the transport and temperature estimates. 

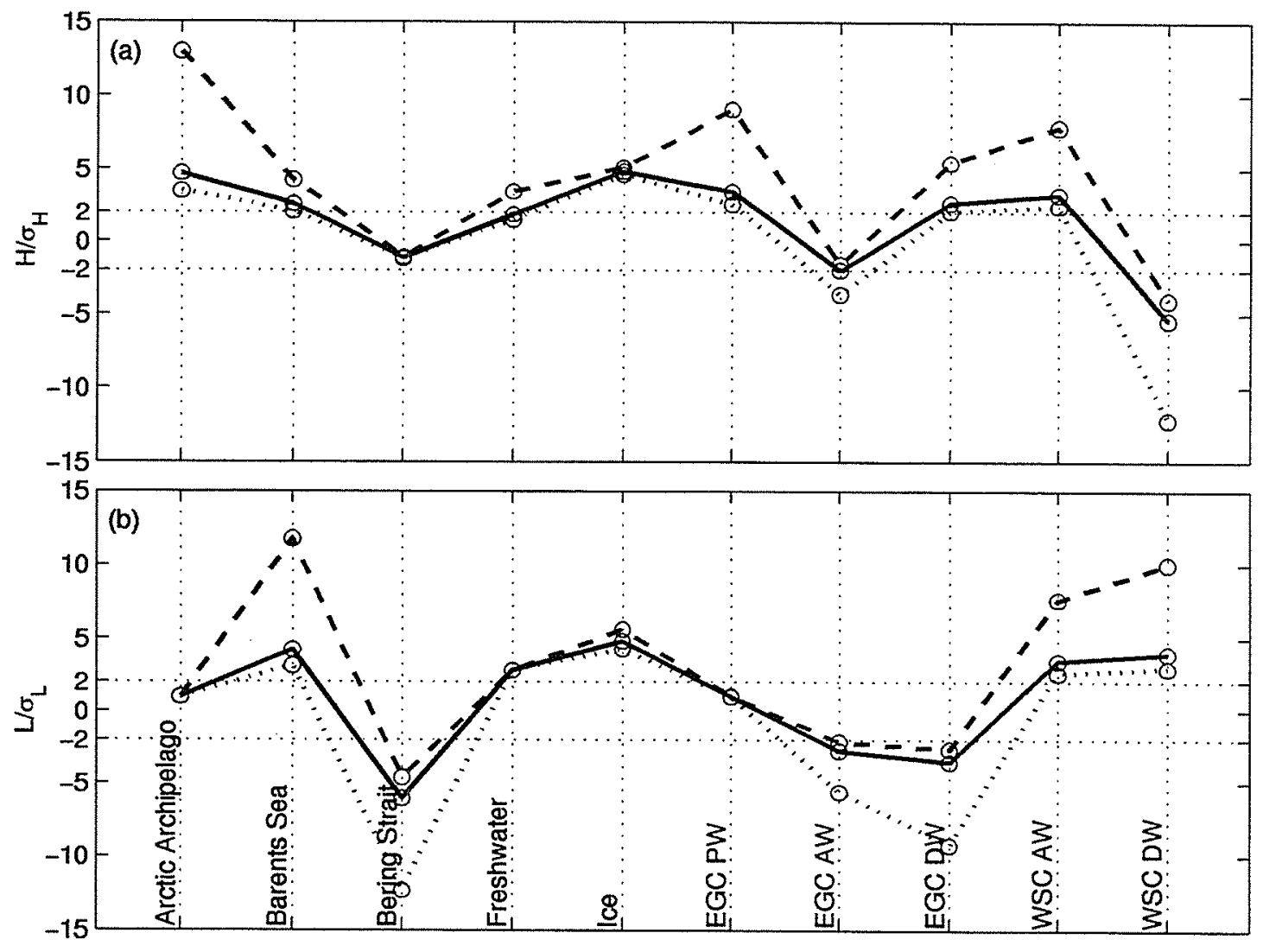

Figure 3.3: (a) $H / \sigma_{H}$ for the 10 Arctic boundary flows, for $\rho_{Q T}=0.9$ (dotted), $\rho_{Q T}=0$ (solid), and $\rho_{Q T}=-0.9$ (dashed). (b) Same for $L / \sigma_{L}$.

For all of the present estimates, both $\bar{Q}$ and $\bar{T}$ are greater than one, so the effect of $\rho_{Q T}$ is palpable but not dominant: on one hand, $H / \sigma_{H}$ can vary by a factor of 4 depending on the value of $\rho_{Q T}$; on the other hand, the significance of the estimates-whether they are more than two standard errors from zero-does not change as $\rho_{Q T}$ is varied (Figure 3.3a).

Discussion for the salinity anomaly flux is again parallel to that for the potential temperature flux, and the results are again similar: as $\rho_{Q S}$ changes, the relative certainty $L / \sigma_{L}$, varies by as much as a factor of 4 , but whether or not 
any of the aggregate estimates is significantly different from zero does not change (Figure 3.3b). ${ }^{4}$

\subsection{Budgets}

This section turns from considering individual flows to considering how they add up in budgets. The first budget is simply the sum of the aggregate estimates derived in the last section. Next, those estimates are revised to conserve mass. Finally, the estimates are further revised to conserve mass, heat, and salt.

\subsubsection{Budget 1: Aggregate estimates only}

The totals of the aggregate estimates are presented in Table 3.2. In summing the flows, error correlations between flows have been ignored. These correlations are probably not exactly zero, but it is hoped that there is enough independent information in the aggregate estimates that the correlations between them are negligible. For example, Rudels (1987) uses mass conservation to determine the Fram Strait transport, so if he has overestimated the Barents Sea inflow, then he has also overestimated the East Greenland Current outflow. Since the aggregate esti-

\footnotetext{
${ }^{4} \mathrm{By}$ reasoning exactly analogous to that used to derive (3.12), the relative certainty of the salinity flux is given by

$$
\frac{L}{\sigma_{L}}=\frac{\bar{Q} \bar{S}+\rho_{Q S}}{\left(\bar{Q}^{2}+\bar{S}^{2}+2 \bar{Q} \bar{S} \rho_{Q S}+2 \rho_{Q S}^{2}\right)^{1 / 2}}
$$

where $\bar{S}=S / \sigma_{S}$. For river runoff, however this is invalid. Since the transport salinity is known exactly, $\sigma_{S}=0$, and $\bar{S}$ is undefined. The relative uncertainty for runoff is thereby derived as follows. Using $\sigma_{S}=0$ gives, by analogy with (3.3),

$$
L=Q S+\left\langle Q^{\prime} S^{\prime}\right\rangle=Q S,
$$

and by analogy with (3.7),

$$
\sigma_{L}^{2}=S^{2} \sigma_{Q}^{2}
$$

Combining these yields $L / \sigma_{L}=Q / \sigma_{Q}$. In short, since the transport salinity of freshwater is a known constant and not a measured (random) quantity, the relative certainty (or lack thereof) for the salt flux is exactly that of the volume transport.
} 
mates include his estimates, they are probably similarly correlated. However, the aggregate estimates also include Blindheim's (1989) estimate for the Barents, and Foldvik et al.'s (1988) estimates for the East Greenland Current. These studies are certainly independent of one another, and their presence in the aggregate estimates weakens the correlations introduced by the inclusion of Rudels's estimates.

The net fluxes are not zero, but all three (volume, temperature, salinity) are less than two standard errors from zero, suggesting that the discrepancies can be explained by errors in the aggregate estimates and do not necessarily indicate changes over time in the total mass, heat, or salt content in the Arctic. Two things must be kept in mind, however.

First, as mentioned just above, several of the contributing studies (Aagaard and Carmack, 1989; Aagaard and Greisman, 1975; Bönisch and Schlosser, 1995; Mauritzen, 1996b; Rudels, 1987) made conservation assumptions when estimating the fluxes. Therefore, the budgets of Table 3.2 do not independently test the hypothesis that the Arctic is in balance. They do show that all previous estimates, some of which assumed conservation and some of which did not, are consistent with each other; nonetheless, the correlations between flows which I hope are small may not be. If they are not, the calculated uncertainties of the aggregate estimates will be too small. To determine the effects of such an error on the calculations in this thesis, additional calculations are performed with larger uncertainty values. These are presented in Section 4.4.8.

Second, even the minimal uncertainties considered here are large enough to make rejection of the steady hypothesis extremely difficult. For example, a net inflow equivalent to a sea-level rise of $8 \mathrm{~m} \mathrm{yr}^{-1}$ would still be "balanced" within two standard errors. This does not indicate failure of the estimation method; rather, it reflects the absence from the calculations of any statement that the mass contained in the Arctic system is expected to be constant. Such a statement is included in the next budget. 


\subsubsection{Budget 2: Aggregate estimates with mass conserva- tion}

As a statement of belief or a theoretical premise, one may wish to stipulate that the net inflow to the Arctic is in fact zero, or very near it. A natural procedure is to revise the aggregate estimates, within their error bars, to achieve a zero sum. The uncertainties of the aggregate estimates allow this to be done systematically, such that fluxes with larger uncertainties receive larger adjustments.

The revisions are calculated by least-squares. The new flux estimates are constrained to be as close as possible to the aggregate estimates, while keeping their sum as close as possible to zero. Thus one seeks revised estimates $\tilde{Q}$ of the true values $\mathcal{Q}$ such that

$$
\begin{aligned}
\tilde{Q}_{i}+e_{Q_{i}} & =Q_{i}, \quad 1 \leq i \leq 10 \\
\left(\sum_{i=1}^{10} \tilde{Q}_{i}\right)+e_{Q_{c}} & =0
\end{aligned}
$$

where the $e$ 's represent the residuals for each constraint. In the system (3.13) and (3.14), $i$ is an index over the ten fluid transports (Bering Strait, Arctic Archipelago, etc.). The system contains 11 equations in 10 unknowns (the $10 \tilde{Q}_{i}$ 's). The leastsquares solution is the set of $\tilde{Q}_{i}$ 's which minimizes $e_{Q_{c}}^{2}+\sum_{i=1}^{n} e_{Q_{i}}^{2}$, the sum of the squared residuals.

It is both possible and desirable to weight the equations before solving so that the solution prioritizes those equations with smaller uncertainties. The uncertainties of the aggregate estimates are interpreted as prior estimates of the likely size of the residuals. The equations are therefore scaled assuming $\left\langle e_{Q_{i}}^{2}\right\rangle=\sigma_{Q_{i}}^{2}$. The conservation uncertainty $\left\langle e_{Q_{c}}^{2}\right\rangle^{1 / 2}$ is chosen to reflect the acceptable magnitude of a non-zero total ( $0.1 \mathrm{~Sv}$ in the standard calculations; this is varied below). Such a departure accounts for errors such as the differences between mass and volume conservation, or for a slow, small change in sea level. All of the $e$ 's are assumed to 
Table 3.3: Arctic flux estimates with standard errors and covariances, based on previously published estimates and on the assumption that the net volume flux is $0 \pm 0.1 \mathrm{~Sv}$. Aggregate temperature and salinity anomaly flux estimates and associated errors are calculated assuming $\rho_{Q T}=\rho_{Q S}=\rho_{S T}=0$.

Budget 2

\begin{tabular}{|c|c|c|c|c|c|c|c|c|c|}
\hline \multicolumn{10}{|c|}{$\begin{array}{l}\text { Aggregate Estimates } \\
\text { Mass Conservation }\end{array}$} \\
\hline \multirow{7}{*}{$\begin{array}{l}\text { Arctic Arch. } \\
\text { Barents Sea } \\
\text { Bering Strait } \\
\text { Freshwater } \\
\text { Fram Strait: }\end{array}$} & $\tilde{Q}$ & $\sigma_{\tilde{Q}}$ & $\tilde{H}$ & $\sigma_{\tilde{H}}$ & $\tilde{L}$ & $\sigma_{\bar{L}}$ & $\left\langle\tilde{Q}^{\prime} \tilde{H}^{\prime}\right\rangle$ & $\left\langle\tilde{Q}^{\prime} \tilde{L}^{\prime}\right\rangle$ & $\left\langle\bar{H}^{\prime} \tilde{L}^{\prime}\right\rangle$ \\
\hline & \multicolumn{2}{|c|}{$\mathrm{Sv}$} & \multicolumn{2}{|c|}{$\mathrm{Sv}^{\circ} \mathrm{C}$} & \multicolumn{2}{|c|}{$\mathrm{Sv}(\mathrm{psu})$} & $\mathrm{Sv}^{20} \mathrm{C}$ & $\mathrm{Sv}^{2}(\mathrm{psu})$ & $\mathrm{Sv}^{20} \mathrm{C}(\mathrm{psu})$ \\
\hline & -1.43 & 0.21 & 1.11 & 0.23 & 0.91 & 0.90 & -0.04 & -0.03 & 0.02 \\
\hline & 1.50 & 0.26 & 8.97 & 3.45 & 0.90 & 0.21 & 0.40 & 0.04 & 0.24 \\
\hline & 0.81 & 0.12 & -0.38 & 0.33 & -1.55 & 0.26 & -0.01 & -0.03 & 0.01 \\
\hline & 0.12 & 0.04 & 0.61 & 0.33 & -4.21 & 1.52 & 0.01 & -0.07 & -0.34 \\
\hline & & & & & & & & & \\
\hline Ice & -0.07 & 0.01 & 5.74 & 1.17 & 2.15 & 0.44 & -0.02 & -0.01 & 0.51 \\
\hline EGC PW & -1.35 & 0.25 & 1.76 & 0.49 & 0.45 & 0.44 & -0.08 & -0.02 & 0.03 \\
\hline EGC AW & -2.09 & 0.47 & -2.20 & 1.02 & -1.02 & 0.31 & 0.23 & 0.11 & 0.12 \\
\hline EGC DW & -1.02 & 0.20 & 0.73 & 0.27 & -0.53 & 0.14 & -0.03 & 0.02 & -0.01 \\
\hline WSC AW & 2.81 & 0.58 & 6.61 & 1.87 & 1.72 & 0.45 & 0.79 & 0.21 & 0.48 \\
\hline WSC DW & 0.73 & 0.12 & -0.72 & 0.14 & 0.37 & 0.09 & -0.01 & 0.01 & -0.01 \\
\hline Surface Heat Flux: & & & & & & & & & \\
\hline Basin & & & 0.00 & 7.44 & & & & & \\
\hline Ice-free Barents & & & -13.94 & 6.27 & & & & & \\
\hline CB Polynyas & & & -1.34 & 0.47 & & & & & \\
\hline EB Polynyas & & & -0.56 & 0.20 & & & & & \\
\hline Shelf: Other & & & 0.00 & 8.37 & & & & & \\
\hline TOTAL (NET) & 0.01 & 0.10 & 6.39 & 13.49 & -0.80 & 1.98 & & & \\
\hline
\end{tabular}

be mutually independent. For details on the scaling procedure see Wunsch (1996).

The scaled system is then solved by standard techniques (e.g., Wunsch, 1996) to produce the volume-conserving transport estimates $\tilde{Q}$ and their uncertainties $\sigma_{\tilde{Q}}$. These are then combined with the aggregate estimates of $T$ and $S$ as in Section 3.4.2 to obtain mass-conserving estimates $\tilde{H}$ and $\tilde{L}$ of the temperature and salinity flux, as well as their variances and covariances. Table 3.3 presents results for $\left\langle e_{Q_{c}}^{2}\right\rangle^{1 / 2}=0.1 \mathrm{~Sv}$. The net volume flux is reduced nearly to zero. Comparison with Table 3.2 shows that the largest revisions occur in the Atlantic Water transports, because those have the largest uncertainties. The uncertainties of the flux totals are reduced by the additional information provided in the conservation statement. ${ }^{5}$

\footnotetext{
${ }^{5}$ The uncertainties of the totals depend in part on the covariances among the individual terms
} 
As with any least-squares problem, it is important to verify that the solution is consistent with initial assumptions. In this case, the revised flux estimates are all within the errors of the aggregate estimates, and the total mass flux is acceptably close to zero, so there is no inconsistency.

Though the aggregate estimates of different flows were uncorrelated, the revised estimates are correlated through the statement of mass conservation. That correlation is only strong between $\tilde{Q}$ for the EGC AW and $\tilde{Q}$ for the WSC AW, with a correlation coefficient of -0.63 . [Correlation coefficients among different flows are calculated from the least squares problem (3.13)-(3.14) by standard techniques. See Wunsch (1996) for details]. These two flows carry most of the correlation because of their relatively large uncertainties. If the true EGC AW export is at the low end of its likely range (near -1 Sv, e.g., $1 \mathrm{~Sv}$ export) while the true WSC AW import is at the high end of its likely range (near $4 \mathrm{~Sv}$ ), the other transports cannot be near their own aggregate estimates and simultaneously make up the $3 \mathrm{~Sv}$ difference to conserve mass. All other correlation coefficients between different transports have absolute values less than 0.3 .

The new parameter in this calculation is the volume conservation tolerance, $\left\langle e_{Q_{c}}^{2}\right\rangle^{1 / 2}$. If it is lowered from $0.1 \mathrm{~Sv}$ to $0.01 \mathrm{~Sv}$, little changes in the solution. If it is raised to $1 \mathrm{~Sv}$, the net inflow grows to near $1 \mathrm{~Sv}$ as the revised estimates of EGC AW and WSC AW transports approach the aggregate estimates for those flows. Other flows are only slightly affected.

(Priestley, 1981). Covariances among the volume flux estimates $\left(\left\langle\tilde{Q}_{i}^{\prime} \tilde{Q}_{j}^{\prime}\right\rangle, i \neq j\right)$, are found from the least squares system (3.13) and (3.14) by standard techniques (Wunsch, 1996). For the temperature and salt budgets, $\left\langle H_{i}^{\prime} H_{j}^{\prime}\right\rangle=T_{i} T_{j}\left\langle\tilde{Q}_{i}^{\prime} \tilde{Q}_{j}^{\prime}\right\rangle$ and $\left\langle L_{i}^{\prime} L_{j}^{\prime}\right\rangle=S_{i} S_{j}\left\langle\tilde{Q}_{i}^{\prime} \tilde{Q}_{j}^{\prime}\right\rangle, i \neq j$, when $\rho_{Q T}=\rho_{Q S}=\rho_{S T}=0$. 


\subsubsection{Budget 3: Aggregate estimates with mass, heat, and salt conservation}

Having estimated the mass-conserving budget, a logical extension is to derive flux estimates which conserve mass, heat, and salt while staying as near as possible to the aggregate estimates. Specifically, the task is to produce revised estimates $\hat{Q}$, $\hat{H}$, and $\hat{L}$ of $\mathcal{Q}, \mathcal{H}$ and $\mathcal{L}$ such that

$$
\begin{aligned}
\hat{Q}_{i}+e_{Q_{i}} & =Q_{i} \\
\hat{H}_{i}+e_{H_{i}} & =H_{i} \\
\hat{L}_{i}+e_{I_{i}} & =L_{i} \\
\sum_{i} \hat{Q}_{i}+e_{Q_{c}} & =0 \\
\sum_{i} \hat{H}_{i}+e_{H_{c}} & =0 \\
\sum_{i} \hat{L}_{i}+e_{I_{c}} & =0
\end{aligned}
$$

where $i$ indicates the flux component, and the sum of the $e^{2}$ 's is again to be minimized. Because the freshwater salinity flux is perfectly correlated with the volume flux, (3.17) is omitted for the freshwater flux, but the freshwater salinity anomaly flux is still accounted for in the salt conservation equation (3.20). Thus the system (3.15)-(3.20) has 34 unknowns - 5 surface heat flux components, 10 volume fluxes, 10 temperature fluxes, and 9 salinity fluxes-and 37 constraints. As before, the equations are scaled by prior estimates of the variances and covariances before solving. For each flow, the variances and covariances of the aggregate estimates (Table 3.2) are taken as prior estimates of the variances and covariances of $e_{Q_{i}}$, $e_{H_{i}}$ and $e_{L_{i}}$. Values of $\left\langle e_{Q_{c}}^{2}\right)^{1 / 2},\left\langle e_{H_{c}}^{2}\right\rangle^{1 / 2}$, and $\left\langle e_{I_{c}}^{2}\right\rangle^{1 / 2}$ are prescribed to reflect acceptable imbalances, and are presumed not to be correlated to one another or to the errors of equations (3.15)-(3.17).

The scaled system is solved by least squares for $\left\langle e_{Q_{c}}^{2}\right\rangle^{1 / 2}=0.1 \mathrm{~Sv},\left\langle e_{H_{c}}^{2}\right\rangle^{1 / 2}=$ 
Table 3.4: Arctic flux estimates with standard errors and covariances, based on previously published estimates, and on the assumptions that the net volume flux is $0 \pm 0.1 \mathrm{~Sv}$, the net temperature flux is $0 \pm 0.1 \mathrm{~Sv}^{\circ} \mathrm{C}$, and the net salinity anomaly flux is $0 \pm 0.1 \mathrm{~Sv} \cdot \mathrm{psu}$. Aggregate temperature and salinity anomaly flux estimates and associated errors are calculated assuming $\rho_{Q T}=\rho_{Q S}=\rho_{S T}=0$.

Budget 3

\begin{tabular}{|c|c|c|c|c|c|c|c|c|c|}
\hline \multicolumn{10}{|c|}{ Aggregate Estimates } \\
\hline \multirow[b]{3}{*}{ Arctic Arch. } & $\hat{Q}$ & $\sigma_{\hat{Q}}$ & $\hat{H}$ & $\sigma_{\dot{H}}$ & $\hat{L}$ & $\sigma_{\hat{L}}$ & \multirow{3}{*}{$\begin{array}{l}\left\langle\hat{Q}^{\prime} \hat{H}^{\prime}\right\rangle \\
\mathrm{Sv}^{20} \mathrm{C} \\
-0.04\end{array}$} & \multirow{3}{*}{$\begin{array}{c}\left\langle\hat{Q}^{\prime} \hat{L}^{\prime}\right\rangle \\
S v^{2}(\mathrm{psu})\end{array}$} & $\left\langle\hat{H}^{\prime} \hat{L}^{\prime}\right\rangle$ \\
\hline & \multicolumn{2}{|c|}{$\mathrm{Sv}$} & \multicolumn{2}{|c|}{$\mathrm{Sv}^{\circ} \mathrm{C}$} & \multicolumn{2}{|c|}{$\mathrm{Sv}(\mathrm{psu})^{2}$} & & & $\mathrm{~Sv}^{2 \circ} \mathrm{C}(\mathrm{psu})$ \\
\hline & -1.43 & 0.21 & 1.11 & 0.22 & 1.07 & 0.77 & & & 0.02 \\
\hline Barents Sea & 1.49 & 0.26 & 8.54 & 3.50 & 0.90 & 0.22 & 0.38 & 0.04 & 0.22 \\
\hline Bering Strait & 0.80 & 0.12 & -0.38 & 0.34 & -1.53 & 0.26 & -0.01 & -0.03 & 0.01 \\
\hline Freshwater & 0.11 & 0.03 & 0.54 & 0.29 & -3.71 & 0.95 & 0.00 & -0.03 & -0.13 \\
\hline Ice & -0.07 & 0.01 & 5.80 & 1.14 & 2.18 & 0.43 & $-00 ?$ & $-0,01$ & 0.48 \\
\hline EGC PW & -1.35 & 0.25 & 1.76 & 0.47 & 0.49 & 0.40 & -0.08 & -0.02 & 0.03 \\
\hline EGC AW & -2.07 & 0.47 & -2.20 & 0.89 & -1.01 & 0.29 & 0.23 & 0.11 & 0.11 \\
\hline EGC DW & -1.02 & 0.20 & 0.73 & 0.26 & -0.52 & 0.14 & -0.03 & 0.02 & -0.01 \\
\hline WSC AW & 2.83 & 0.57 & 6.54 & 2.22 & 1.77 & 0.51 & 0.76 & 0.19 & 0.45 \\
\hline WSC DW & 0.73 & 0.12 & -0.73 & 0.14 & 0.37 & 0.10 & -0.01 & 0.01 & -0.01 \\
\hline Surface Heat Flux: & & & & & & & & & \\
\hline Basin & & & -1.97 & 6.22 & & & & & \\
\hline Ice-free Barents & & & -15.34 & 5.56 & & & & & \\
\hline CB Polynyas & & & -1.35 & 0.47 & & & & & \\
\hline EB Polynyas & & & -0.56 & 0.20 & & & & & \\
\hline Shelf: Other & & & -2.49 & 6.59 & & & & & \\
\hline TOTAL (NET) & 0.01 & 0.10 & 0.00 & 0.10 & 0.00 & 0.10 & & & \\
\hline
\end{tabular}

$0.1 \mathrm{~Sv}^{\circ} \mathrm{C}$, and $\left\langle e_{I_{c}}^{2}\right\rangle^{1 / 2}=0.1 \mathrm{~Sv} \cdot$ psu. Resulting estimates $\hat{Q}, \hat{H}$ and $\hat{L}$ are presented with their variances and covariances in Table 3.4. As required, the net fluxes vanish. Most of the estimates are close to those produced by mass conservation alone, and as before, exceptions occur where large uncertainties allow large adjustments: the salinity fluxes of the Arctic Archipelago and the freshwater input, and the surface heat flux terms.

Checking for consistency with prior assumptions, the conservation conditions are satisfied and the new estimates are within the errors of the aggregate estimates. One other prior assumption was made: the aggregate $H$ 's and $L$ 's were formed assuming the transport temperatures and salinity anomalies are given by $T$ and 
$S$. Dividing the $\hat{H}$ 's and $\hat{L}$ 's by the $\hat{Q}$ 's gives revised transport temperatures and salinity anomalies well within the error bars of the aggregate $T$ 's and $S$ 's. So the mass, heat, and salt-conserving estimates are consistent with prior assumptions.

The revised estimates are again correlated through the conservation statements (3.18)-(3.20), and the principal correlations are again among the most uncertain fluxes. The error in the Arctic Archipelago salinity flux is positively correlated (0.62) with that of the freshwater volume flux estimate, and negatively with the freshwater salinity flux estimate. If these errors do not vary together, salt conservation and the aggregate salt flux estimates cannot simultaneously be respected. Likewise, if heat is to be conserved in the system, the surface heat flux estimates for the ice-free Barents Sea, the basin, and the unknown shelf terms cannot all have large errors in the same direction. Those estimates are therefore negatively correlated (basin-Barents, -0.34 ; basin-shelf, -0.51 ; shelf-Barents, -0.41 ). The low uncertainties of the total mass, heat, and salt budgets in Table 3.4 for the system are due to these correlations.

The terms of all three budgets are plotted together for comparison in Figure 3.4.

\subsection{Discussion}

While the calculations of the various uncertainties and covariances are not very difficult, simpler approaches are possible. It is probably easiest to estimate the heat flux and salt flux uncertainties by $T \sigma_{Q}$ and $S \sigma_{Q}$, ignoring the contribution of the uncertainties of $T$ and of $S$. How much difference would that make? From (3.7), the uncertainty of $T$ is important when $Q^{2} \sigma_{T}^{2} / T^{2} \sigma_{Q}^{2}$ is $O(1)$ (for $\rho_{Q T}=0$ ). The final importance of using the full expression (3.7) can be judged by the difference between the flux uncertainties from Table 3.4, and the simple estimates $T \sigma_{Q}$ and $S \sigma_{Q}$. The two results are compared for the various flows in Table 3.5. For most 

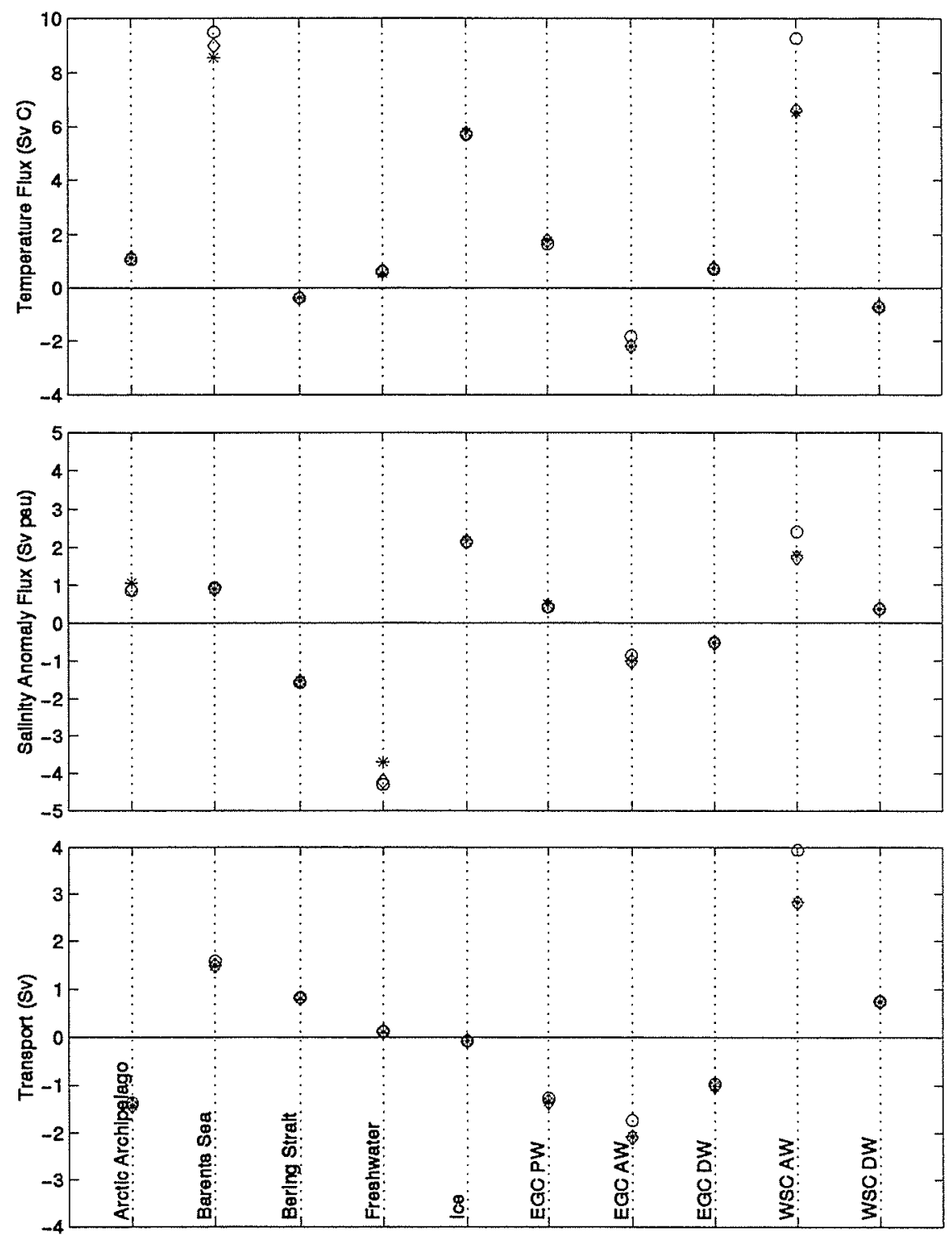

Figure 3.4: Temperature, salinity anomaly, and volume fluxes for each of the 10 boundary flows. Aggregate estimates (Table 3.2) are represented by o, massconserving estimates (Table 3.3) by $\diamond$, and mass-, heat-, and salt-conserving estimates (Table 3.4 ) by *. Positive values represent fluxes into the Arctic. The temperature fluxes are balanced in steady state by the surface heat flux (not shown). 
Table 3.5: Heat and salt flux uncertainties from Table $3.4 \sigma_{\tilde{H}}$ and $\sigma_{\tilde{L}}$ compared to simpler uncertainty estimates $T \sigma_{Q}$ and $S \sigma_{Q}$.

\begin{tabular}{lcccc}
\hline & $\sigma_{\tilde{H}}$ & $T \sigma_{Q}$ & $\sigma_{\tilde{L}}$ & $S \sigma_{Q}$ \\
& \multicolumn{2}{c}{$\mathrm{Sv}^{\circ} \mathrm{C}$} & \multicolumn{2}{c}{$\mathrm{Sv} \cdot \mathrm{psu}$} \\
\hline Arctic Arch. & 0.22 & 0.17 & 0.77 & 0.14 \\
Barents Sea & 3.50 & 1.58 & 0.22 & 0.16 \\
Bering Strait & 0.34 & 0.06 & 0.26 & 0.23 \\
Freshwater & 0.29 & 0.22 & 0.95 & 1.52 \\
Fram Strait: & & & & \\
Ice & 1.14 & 1.17 & 0.43 & 0.44 \\
EGC PW & 0.47 & 0.34 & 0.40 & 0.09 \\
EGC AW & 0.89 & 0.55 & 0.29 & 0.26 \\
EGC DW & 0.26 & 0.14 & 0.14 & 0.10 \\
WSC AW & 2.22 & 2.21 & 0.51 & 0.58 \\
WSC DW & 0.14 & 0.12 & 0.10 & 0.06 \\
\hline
\end{tabular}

fluxes, the two approaches give nearly the same uncertainty. But for a few, such as the salt flux through the Arctic Archipelago or the Bering Strait heat flux, the carefully calculated uncertainty is over five times greater than the rough estimate.

Another calculation one might be tempted to skip is the correlation between transports, heat fluxes, and salt fluxes. For many applications this information is not needed. But if mass, heat and salt conservation are all used to constrain a model, as they are in subsequent chapters of this thesis, then it is important to know whether those constraints are independent or not. Table 3.6 shows the correlation coefficients for the flux estimates of Table 3.4. The correlations between the transport, heat flux, and salt flux for each flow are often large, and ignoring them would lead to errors in the model solution.

Correlations among the different flows are also of importance. The correlations among flows introduced by the requirements of mass, heat, and salt conservation indicate the terms of the budgets with the greatest uncertainty. One way to look at those correlations is to examine subtotals of the fluxes. If the estimates are not constrained to conserve mass, the sum of the aggregate transport estimates of all 
Table 3.6: Coefficients of correlation between volume $(Q)$, heat $(H)$ and salt $(L)$ fluxes for each of the flows estimated in Table 3.4.

\begin{tabular}{rccc}
\hline & $\rho_{Q H}$ & $\rho_{Q L}$ & $\rho_{H L}$ \\
\hline Arctic Arch. & -0.75 & -0.12 & 0.09 \\
Barents Sea & 0.42 & 0.69 & 0.29 \\
Bering Strait & -0.16 & -0.87 & 0.14 \\
Freshwater & 0.48 & -1.00 & -0.48 \\
Fram Strait: & & & \\
Ice & -1.00 & -0.99 & 0.99 \\
EGC PW & -0.70 & -0.19 & 0.13 \\
EGC AW & 0.56 & 0.80 & 0.45 \\
EGC DW & -0.55 & 0.72 & -0.40 \\
WSC AW & 0.60 & 0.66 & 0.40 \\
WSC DW & -0.84 & 0.62 & -0.52 \\
\hline
\end{tabular}

oceanic fluxes except the EGC and WSC Atlantic Water fluxes is $-0.38 \pm 0.51$ Sv. If the aggregate estimates are correct and mass is to be conserved, the two remaining transports must balance the total. Considering that the aggregate estimates for the Atlantic Waters sum to $2.20 \pm 1.08 \mathrm{~Sv}$, this restriction removes much of these two flows' independence. Likewise, the net oceanic heat flux is $25.56 \pm 4.91 \mathrm{~Sv}^{\circ} \mathrm{C}$, and the requirement that the surface heat flux balance it is much more restrictive than the "direct" surface heat flux estimates alone, which total $-15.84 \pm 12.85 \mathrm{~Sv}^{\circ} \mathrm{C}$.

Subtotals of the aggregate estimates also give a gross indication of what is happening in the Arctic interior. Separating the fluxes into those that interact directly with the deep basins (Fram Strait and the basin surface heat flux) and those that interact with the shelves (all others) gives a shelf total of $Q_{\text {net }}=$ $1.17 \pm 0.37 \mathrm{~Sv}$, which must be balanced by net mass flux from the shelves to the basin. Further, $L_{\text {net }}$ for the shelves is $-4.06 \pm 1.78 \mathrm{~Sv} \cdot$ psu, while for the basin it is $+4.01 \pm 0.99 \mathrm{~Sv} \cdot$ psu. In other words, external sources tend to freshen the shelves, and increase the salinity of the basins. To maintain steady state in both areas, the overall effect of shelf-basin exchange must be to transport fresh water from the shelves and/or saline water to the shelves. It must be kept in mind, however, 
that this is a net result. It is the sum of processes which further this end, such as shelfbreak upwelling of saline water (Aagaard and Roach, 1990) and surface layer transport of ice and freshwater to the basin (Schlosser et al., 1994), and processes which work against it, such as dense, saline overflows from the shelves to the basin, and ice drift from the basin to the shelves.

The procedures and conclusions of this project rest on the assumption of steady state. If some of the previous flux uncertainties are wrong, the budgets probably still describe the likely values of $\mathcal{Q}, \mathcal{H}$ and $\mathcal{L}$. But if the true state of the Arctic can not be represented by these constant values then the simultaneous combination of measurements collected over many years is invalid. Even in this case, however, the steady state assumption is still useful as a theoretical simplification.

The calculation of the aggregate heat and salt transport estimates-i.e., extracting transport temperatures and salinities from each study to determine their uncertainty, then folding them back in to obtain transports-is cumbersome, and would not be necessary if variance and covariance information were provided with published transport estimates. Estimating the variances and covariances is difficult, but it has been shown here that carrying out their calculation as far as possible in terms of clearly defined known and unknown factors can be fruitful. Error information is crucial if flux estimates are to be quantitatively compared to or combined with any other information at all.

\subsection{Summary}

This chapter

1. provided rough estimates of Arctic boundary fluxes and their uncertainties based on available published estimates, 
2. used the uncertainties for combining previous flux estimates into more reliable aggregates, and for combining flux estimates with other information such as conservation statements, and

3. expressed the uncertainty in terms of known quantities (such as variances) and unknown ones (such as correlation coefficients) to bound the uncertainty and examine quantitatively the dependence on the unknowns.

Available previous estimates were shown to be consistent among themselves and with the conservation of mass, heat, and salt in the Arctic. The derived aggregate estimates were more accurate than previous results, owing to the combination of estimates which they comprised, and they were not terribly sensitive to unknown parameters. The resulting budgets indicated the necessity, in steady state, of net mass flux from the shelves to the basin and a net salt flux from the basin to the shelves. 


\section{Chapter 4}

\section{Estimates of Shelf-Basin}

\section{Exchange}

\subsection{Introduction}

Past models have been judged by whether they can maintain observed temperature and salinity profiles while satisfying conservation constraints (Killworth and Smith, 1984; Björk, 1990). The main point of this chapter is that more stringent criteria are required. To show this, the Arctic circulation, including shelf-basin exchange, is estimated twice: first by inverting mass, temperature, and salinity conservation equations (Section 4.2), and then again, adding conservation statements for $\delta^{18} \mathrm{O}$ (Section 4.3). It will be shown that the $\delta^{18} \mathrm{O}$ constraints drastically reduce the uncertainty of the shelf-basin exchange. Following those two sections, two further sets of calculations will extend the $\delta^{18} \mathrm{O}$-conserving inversions. Section 4.4 examines the assumptions built into the model structure, and Section 4.5 explores what steady solutions would look like if some key Arctic parameters were very different than they are today. 


\subsection{Estimates from Temperature and Salinity}

The model system has 83 unknown fluxes (Figure 4.1) constrained by 70 equations: 18 each of volume, potential temperature, and salinity anomaly conservation, 14 constraints on the total fluxes of the various outflows, and 2 constraints on the volume of the West Spitzbergen Current inflows. The numerical rank of the system is 65 , but the non-negative constraints eliminate the solution nullspace. Because the present focus is on steady solutions, the mass, heat, and salt-conserving estimates discussed in Section 3.5.3 and listed in Table 3.4 are used, except where noted.

\subsubsection{Optimal solution}

The solution of minimum residual norm (Figure 4.1) is the solution which most closely satisfies, in the least-squares sense, the conservation constraints and the constraints on the boundary fluxes from Chapter 3. In other words, it is the "steadiest" solution, given the boundary flux estimates.

In the Canadian Basin, shelf-basin exchange occurs primarily in the surface mixed layer, as approximately $5 \mathrm{~Sv}$ of reciprocal exchange. Only $0.2 \mathrm{~Sv}$ of dense water are formed, all with the density of the lower halocline. In the Eurasian Basin, the dense water flux dominates the flow across the shelf break, with over $2 \mathrm{~Sv}$ of dense water flowing to the upper halocline, and an additional $0.4 \mathrm{~Sv}$ sinking to the Atlantic layer. From the basin, $0.8 \mathrm{~Sv}$ flows from the surface mixed layer water to the shelf, partially replacing the dense water which flows from the shelf.

More dense water production is needed in the Eurasian Basin than in the Canadian, because halocline water flows out of the Eurasian Basin through the East Greenland Current. Dense water from the shelves is a required ingredient in replacing that lost halocline water. In contrast, little to no halocline water is drained directly from the Canadian Basin, so none needs to be replaced. This inequality 


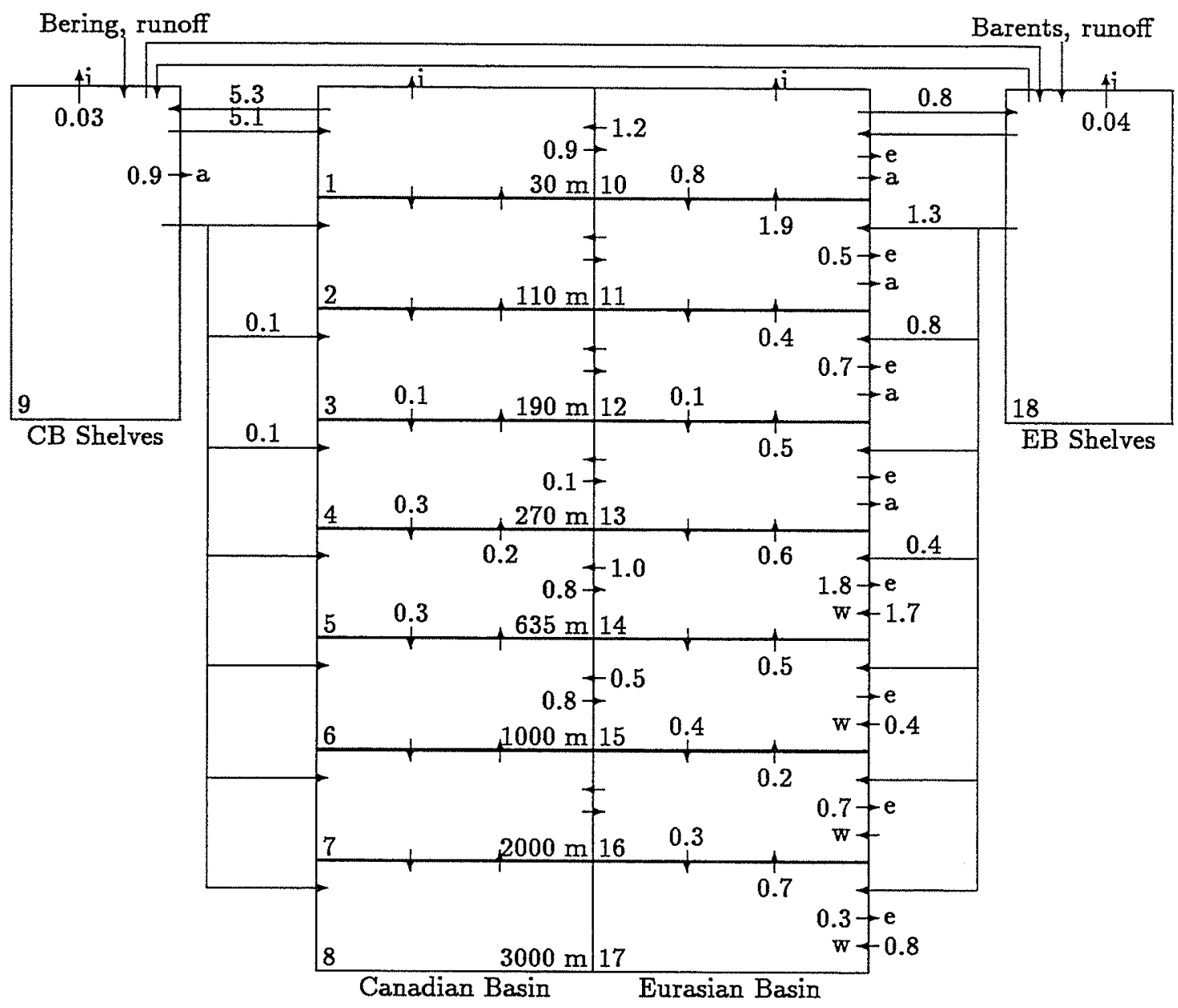

Figure 4.1: Solution of minimum residual norm (i.e., "steadiest" solution) for the temperature-salinity inversion. Transports in Sverdrups are rounded to the nearest tenth (hundredth for ice); unmarked arrows indicate zero or near zero transport. Water removed as ice is indicated by $i$, outflows through the Arctic Archipelago and East Greenland Current by a and e, and West Spitzbergen Current inflows by $w$. 


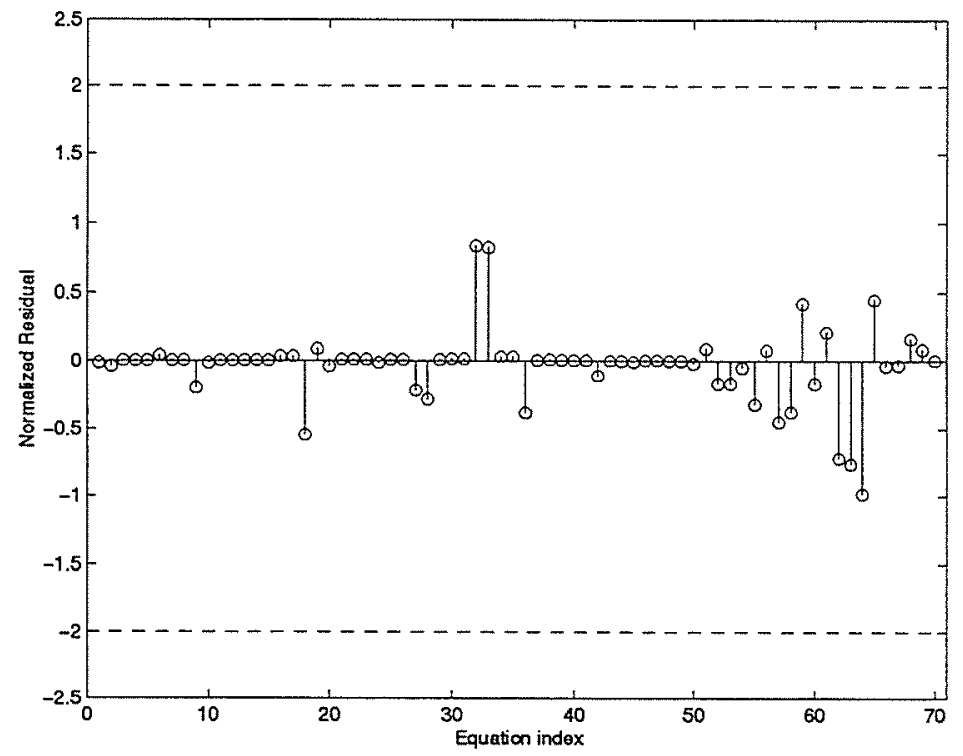

Figure 4.2: Equation residuals for the optimal solution of the temperature-salinity inversion, each normalized by the standard error of the equation.

persists throughout the calculations of this study.

As shown in Figure 4.2, the residuals are small; in no case greater than one standard error in magnitude. The model has sufficient freedom to fit the constraints.

Other aspects of the solution could be discussed, such as the maintenance of the Canadian Basin surface mixed layer by diffusion with the shelf and with the Eurasian Basin. Such discussions are better postponed, however, since the high uncertainty of the solution precludes many firm conclusions. This is shown in the next section.

\subsubsection{Uncertainty of optimal solution}

The least-squares solution depends directly on the values prescribed for the boundary fluxes, and those values are not known precisely. Instead, they are believed 
likely to fall within a certain range, calculated in Chapter 3. There is therefore a corresponding range of least-squares solutions, and the size of that range is the uncertainty of the optimal solution.

To estimate the uncertainty, 1000 Monte Carlo trials were calculated as described in Section 2.7.3. This produced, for each unknown flux, a sample distribution of transports. Figure 4.3 describes for each flux the distribution of Monte Carlo transports with a series of three numbers: low mode high. Mode is the most common value for the transport. Low and high are the ends of the $95 \%$ confidence interval for the transport, i.e., they are the 26th and 975th largest transports seen in 1000 trials. Transports whose low value is zero may therefore be viewed as not significantly different from zero. ${ }^{1}$ That this is the case for nearly all the fluxes highlights the difficulty of looking at each unknown in isolation: in any given trial, any given "pipe" might not carry any flow, but in every trial there is flow somewhere.

It is sometimes more helpful to consider the mode. In several cases, the mode is also zero. While it is natural to expect the mode to lie exactly in the middle of the $95 \%$ interval, it must be kept in mind that the solution is not Gaussian. The Monte Carlo perturbations to the forcing are Gaussian, but the non-negative constraints make the model system non-linear, producing solution distributions which can be highly skewed. A distribution such as $0.00 .026 .9 \mathrm{~Sv}$, for example, indicates a flux that was rarely used, but which was occasionally very high. The non-linearity of the system also means that the modal value of each transport does not necessarily match the central value of the previous section. The previous section showed the most likely transport for the single most likely value of the boundary fluxes. The

\footnotetext{
${ }^{1}$ For example, a typical distribution of 1000 realized transports for a given unknown might include several hundred zeros, with the remainder of the realizations ranging between zero and some maximum (usually 1-2 Sv). Each realization with zero transports indicates that the mathematical least-squares problem, divorced from physical interpretation, has an optimal solution with negative transports. But non-negative constraints have been imposed, making the model physically relevant, and the solution of minimal residuals which meets the non-negative constraints is derived by setting those elements which would have been negative to zero, and then adjusting the other elements of the solution to reduce the residual as much as possible.
} 


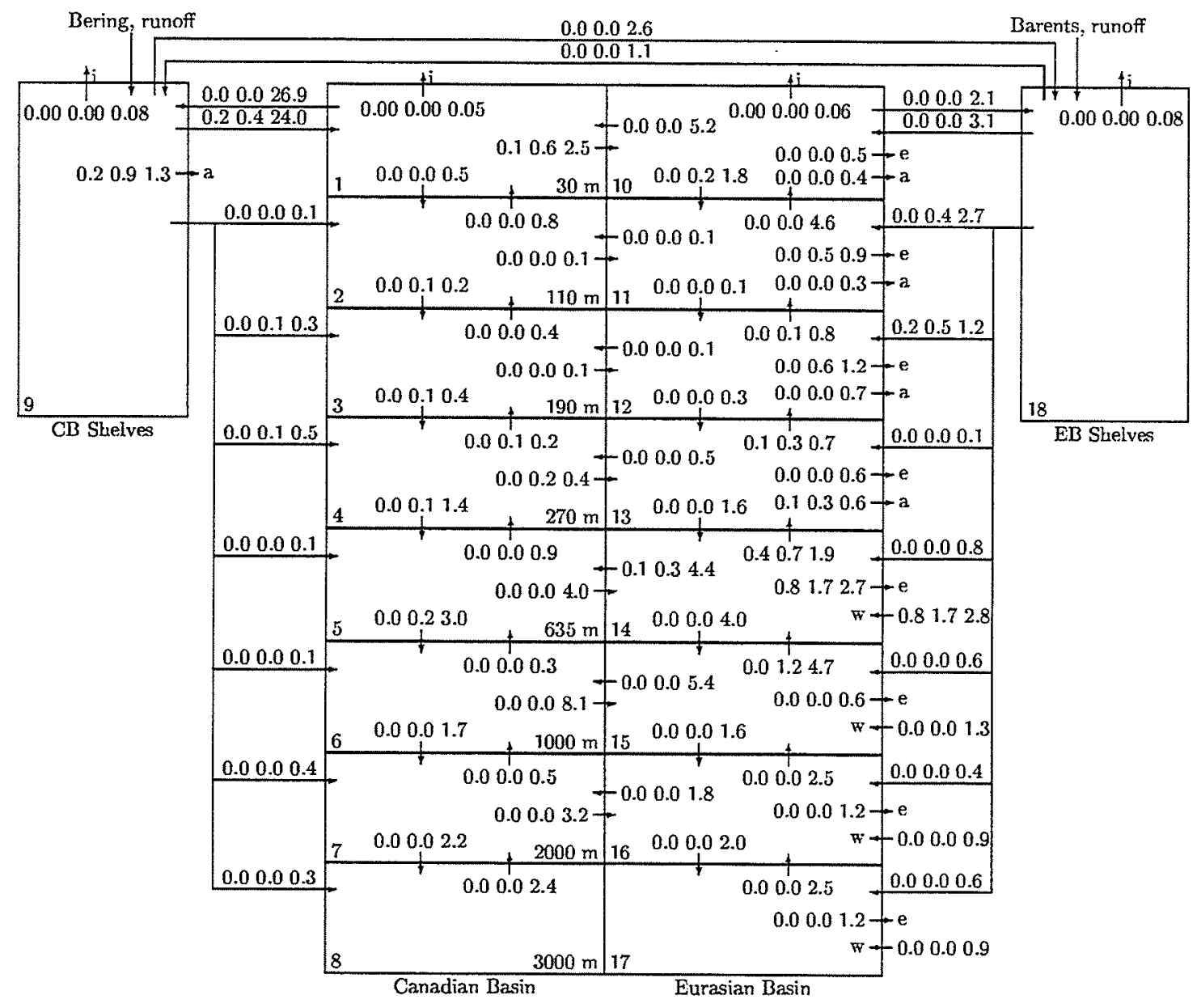

Figure 4.3: Monte Carlo transport distributions for each flux in the temperaturesalinity inversion. Each distribution is represented in Sverdrups as low mode high, where low and high are the lower and upper limits of the Monte Carlo $95 \%$ interval, and mode is the most likely value. Blank arrows indicate paths unused in the solution in at least $95 \%$ of the trials (i.e., 0.00 .00 .0 ). Outflows and ice production are marked as in figure 4.1 . 
mode, on the other hand, gives the most likely transport for that flux over the ensemble of plausible boundary fluxes. Since the mode represents the ensemble, information from each particular realization is lost; the modes of the fluxes into a particular box may not sum to zero, though the fluxes did sum to zero in each of the thousand realizations.

Using cross-correlations, the singular value decomposition, and other tools, attempts were made to identify circulation schemes into which the 1000 realizations could be grouped. The hope was to discover the most likely scheme, as well as alternatives which would occur when forced by particular combinations of boundary fluxes. These attempts were foiled by the rich variety of solutions which appeared in the Monte Carlo trials. They did, however, uncover subgroups of fluxes whose transports varied together. An example follows.

The amount of surface mixed layer exchange in the Canadian Basin is wildly uncertain: though its most likely (mode) value is small, its $95 \%$ confidence interval includes exchanges as high as $24 \mathrm{~Sv}$ (Figure 4.3). These surface fluxes to and from the shelf vary together and are nearly always equal and opposite, representing diffusion between the basin and the shelf rather than net advection from one to the other. (These large flows are created to balance surface heat fluxes. Since the temperature difference between the surface boxes is small, significant heat transfer can only occur if the volume exchanged is large. See below for more details.) The total deep water formation, shown in Figure 4.4, is also much more uncertain than the estimates of previous studies. The most likely value of total production is $1.9 \mathrm{~Sv}$, similar to previous results, but the $95 \%$ interval for that value is $1.2-4.6 \mathrm{~Sv}$. The variation is almost entirely in the dense water production of the Eurasian Basin shelves (Figure 4.5), most of which flows to the upper halocline.

These two highly variable fluxes-Canadian Basin surface mixed layer exchange and dense water flux from the Eurasian Basin shelves to the upper haloclineare tightly correlated to one another. They are also correlated to the rest of the 


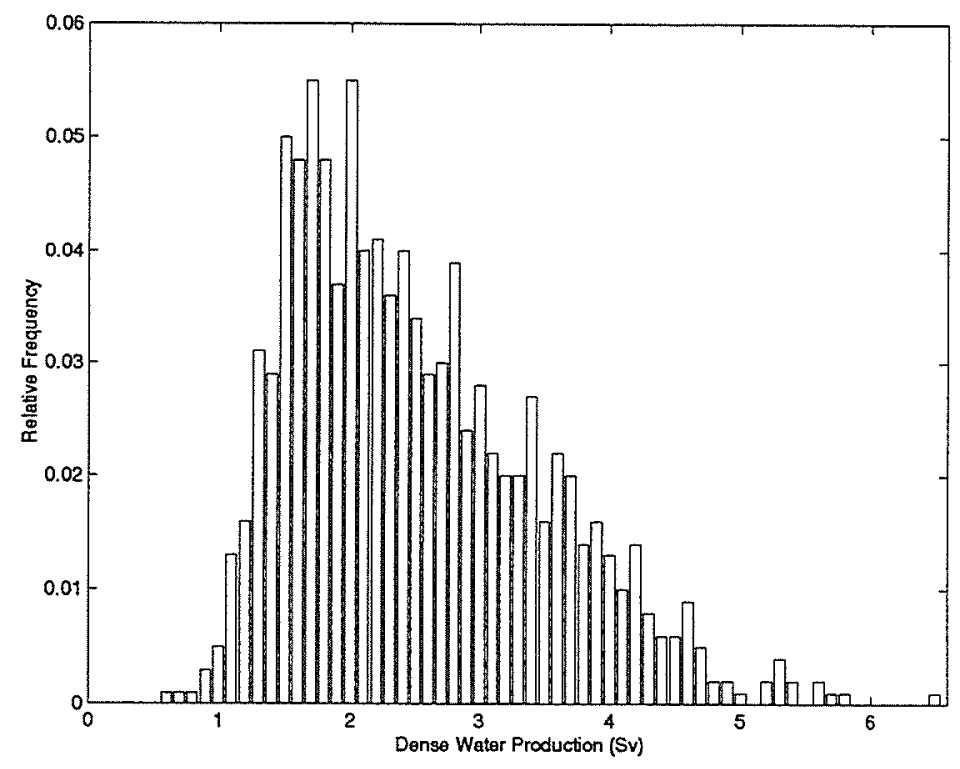

Figure 4.4: Histogram of 1000 Monte Carlo realizations of total dense water production in the temperature-salinity calculation.

circulation among surface boxes, and to the vertical exchange between the Eurasian Basin upper halocline and the surface mixed layer. The large uncertainty in the exchanges through this surface network is a direct result of the large uncertainty in the boundary fluxes. Specifically, the variation in shelf-surface mixed layer exchange is most strongly correlated to variations in the surface heat fluxes on the Canadian Basin shelves (correlation coefficient -0.66) and in the Canadian Basin itself (0.55). Any strong heat flux affecting one box or the other is balanced by mixing between the two, and the temperature difference between them $\left(0.3^{\circ} \mathrm{C}\right)$ is small enough that vigorous mixing is required to balance large surface fluxes. The mixing also carries a salt flux, which must in turn be balanced. This is accomplished through exchanges between the $\mathrm{CB}$ and $\mathrm{EB}$ shelves, which requires advection to or from the Eurasian Basin shelf box. This mass forcing is compensated by variations in the amount of dense water flowing to the upper halocline.

More simply, the rigid framework of the steady-state assumption links the sur- 


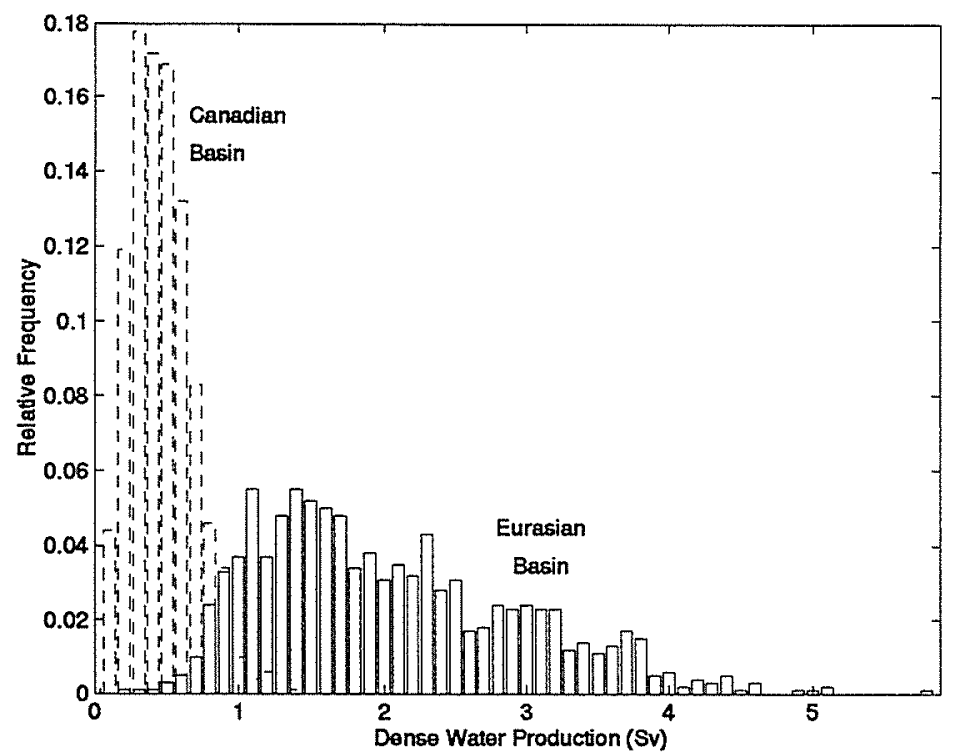

Figure 4.5: The same Monte Carlo realizations of dense water production shown in Figure 4.4, but with production on Canadian Basin shelves separated from that the Eurasian Basin.

face heat flux on the Canadian Basin shelves to dense water production on the Eurasian Basin shelves. The surface heat flux is uncertain enough that large fluxes are possible, and in a system with small temperature differences, large heat fluxes must be balanced by large transports. This link, and the uncertainty in its forcing, are the dominant causes of the uncertainty of the shelf-basin exchange in the steady model.

The Monte Carlo trials also give 1000 realizations of the residuals for each equation. Their 95\% confidence intervals and modes are plotted in Figure 4.6. The model consistently fits the data over the entire plausible range of boundary fluxes. 


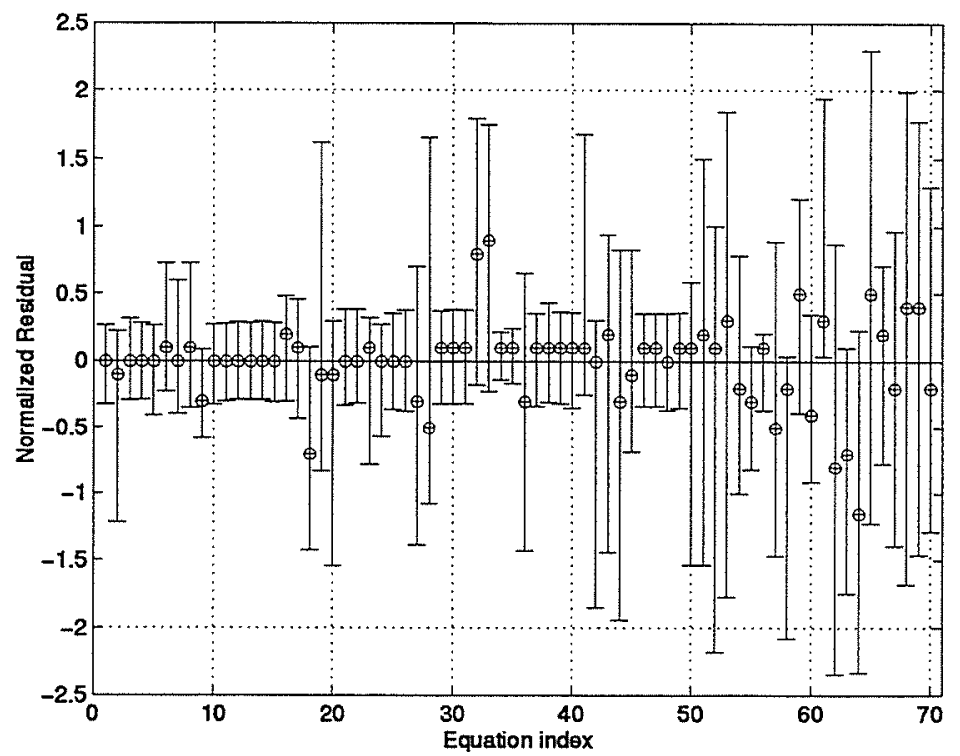

Figure 4.6: Monte Carlo distribution of normalized residuals for the temperature and salinity conservation calculation. All constraints are consistently met within or near two standard errors.

\subsubsection{Extreme solutions}

The linear programming techniques discussed in Section 2.7.4 were used to determine the minimum and maximum dense water production and the minimum and maximum shelf-basin surface mixed layer exchange that could be obtained without violating any constraint by more than 1.5 standard errors. For this purpose, "dense water production" was measured as the sum of the fluxes from the shelf to the lower seven boxes of the basin. "Surface mixed layer exchange" was defined as the sum of the four fluxes linking the shelves to the basin surface boxes. The results confirmed how truly uncertain temperature-salinity inversions of the shelf-basin exchange are, given the uncertainty in the flux data.

The minimum values for the two types of exchange were each zero. In other words, consistent solutions are possible with no dense water production, as long as mass can leave the shelf through the surface mixed layer. Likewise, consistent 
Table 4.1: Extreme values of each type of shelf-basin exchange, consistent with mass, temperature and salinity conservation within 1.5 standard errors. Transports are in Sv.

\begin{tabular}{lcc}
\hline & Min & Max \\
\hline Dense Water Flux & $\mathbf{0 . 0}$ & 9.4 \\
SML Exchange & $\mathbf{0 . 0}$ & 72.2 \\
\hline
\end{tabular}

solutions are possible with no surface mixed layer transport, as long as mass can leave the shelf as dense water. Thus, the type of cross-shelfbreak flow is not determined by the constraints, but there must be some transport across the shelf break because consistent solutions require some way for the surplus flux to the shelves to make its way into the basin, as discussed in Chapter 3 .

To confirm this, an inversion was calculated in which no flow was allowed between the shelves and the basins. The model constraints were violated in two major ways. First, the mass conservation equation for the Eurasian Basin shelf box was violated by approximately five standard deviations: the Barents Sea inflow has nowhere to go. Second, the EGC Polar Water outflow was set nearly to zero, almost three standard errors below the aggregate estimate. This was partly because of simple mass balance considerations: outflow from the basin could not be maintained without compensation from the shelves. Furthermore, Polar Water is particularly hard to replace, so it is less costly for the model to violate the Polar Water outflow condition than to try and replace that water without the shelf contribution. This latter factor is discussed in detail in Section 5.3.

The maximum dense water production still consistent within 1.5 standard errors was 9.4 Sv, accompanied by an enormous 61.5 Sv of SML exchange (Figure 4.7). The maximum surface mixed layer exchange was an even larger $72.2 \mathrm{~Sv}$, and was accompanied by $5.0 \mathrm{~Sv}$ of deep water formation (Figure 4.8) These results are summarized in Table 4.1. 


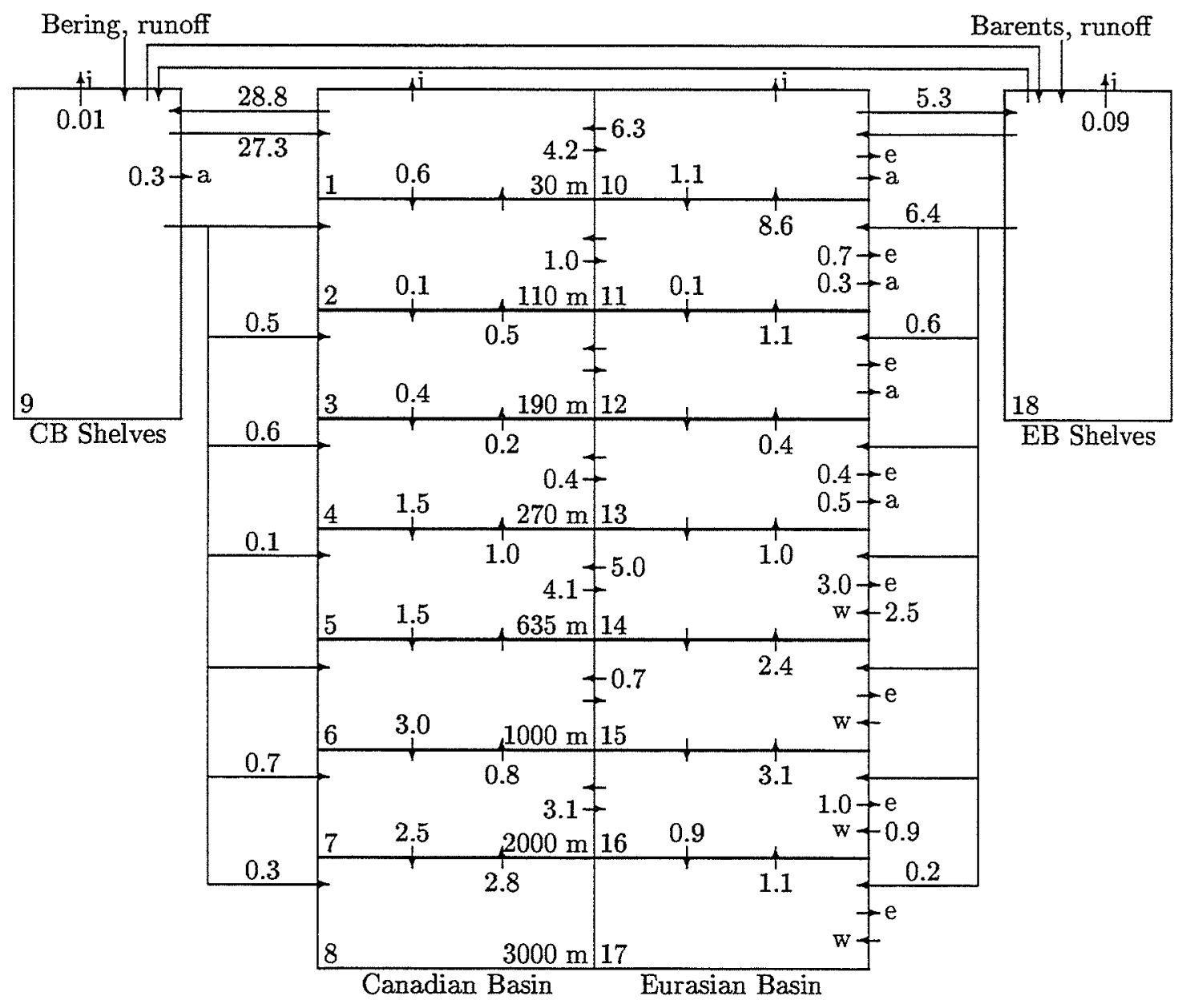

Figure 4.7: Linear programming solution of maximum dense water formation for the temperature-salinity inversion. Transports in Sverdrups are labeled as in Figure 4.1. 


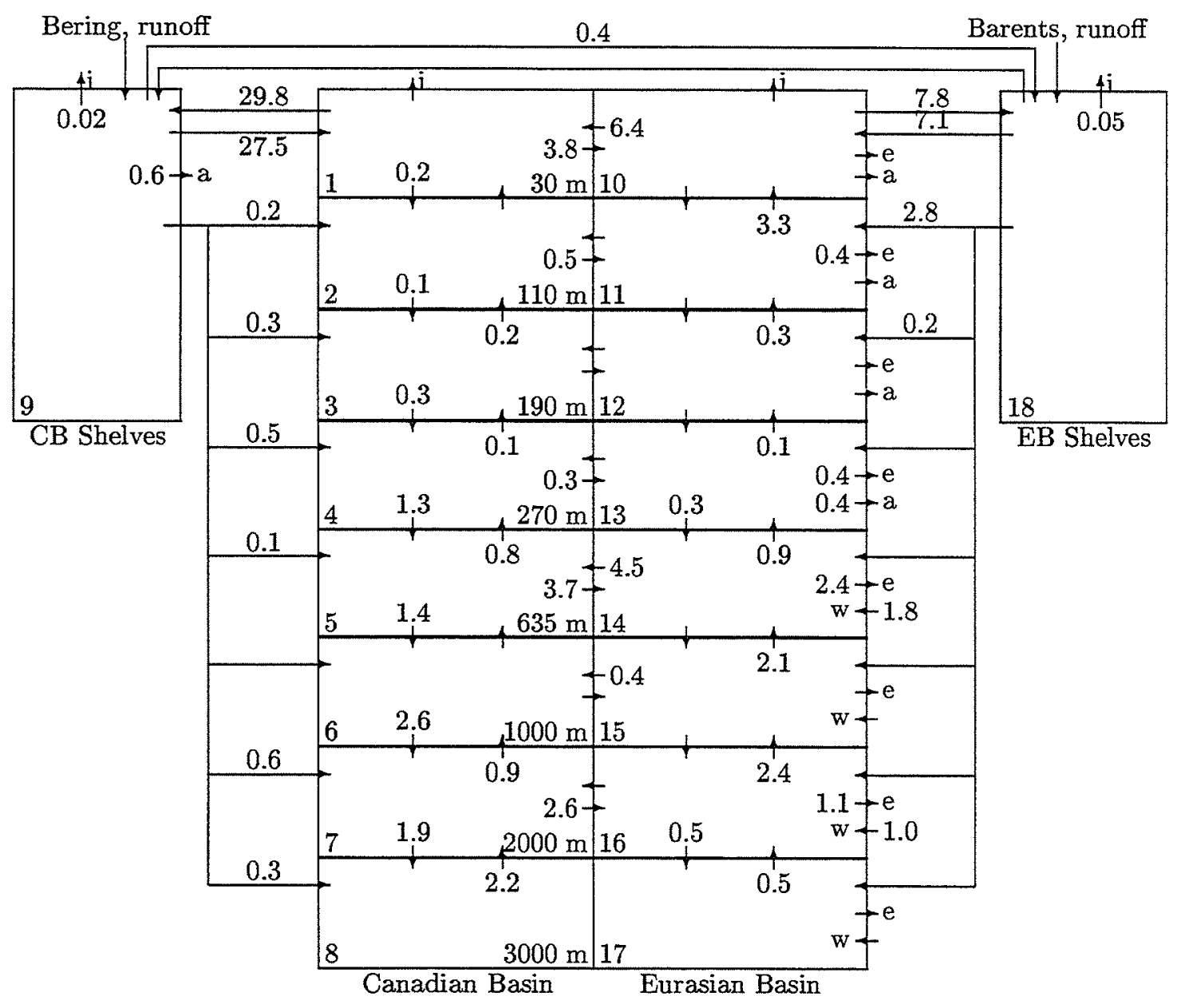

Figure 4.8: Linear programming solution of maximum surface mixed layer exchange. Transports in Sverdrups as in Figure 4.1. 
To summarize, the linear programming calculations confirm that the data used in the temperature-salinity inversions are not sufficient to exclude steady solutions involving very large shelf-basin transports, or even to partition the cross-shelfbreak flow between dense overflows and surface mixed layer drift!

\subsubsection{Sensitivity to prescribed temperatures and salinities}

The sensitivity of these results to the temperatures and salinities prescribed in the basin and on the shelves is now evaluated.

\section{$\operatorname{Basin} \mathrm{T} / \mathrm{S}$}

To test the sensitivity of the temperature-salinity inversions to the prescribed temperatures and salinities in the basins, alternative $T / S$ profiles were used. Based on the temperature and salinity data, "envelope profiles" likely to enclose the unknown, true average profiles were roughly estimated. For each property, four extreme profiles were used: profiles with the maximum and minimum values of the property within the envelope, and profiles with maximum and minimum vertical gradients within the envelope (Figure 4.9). Four test profiles plus the standard profile, for temperature and salinity, provide $25 \mathrm{~T} / \mathrm{S}$ combinations which were inverted as above. Consistent solutions were found in every case, except for those cases with high basin temperatures and low surface salinity. The large residuals in those cases were related to the model's inability to export enough cold Polar Water, that task being made difficult by the combination of a warm basin and a relatively fresh surface mixed layer, where upwelling was more difficult to balance. This phenomenon is examined in detail in Section 5.3.

In the 25 calculations, the lowest mode of the optimal total dense water production was $0.7 \mathrm{~Sv}$, with a $95 \%$ interval of $0.2-1.2 \mathrm{~Sv}$, while the highest was $3.4 \mathrm{~Sv}$, with a $95 \%$ interval of 1.5-8.9 Sv. (Recall that the standard mode was $1.9 \mathrm{~Sv}$, 

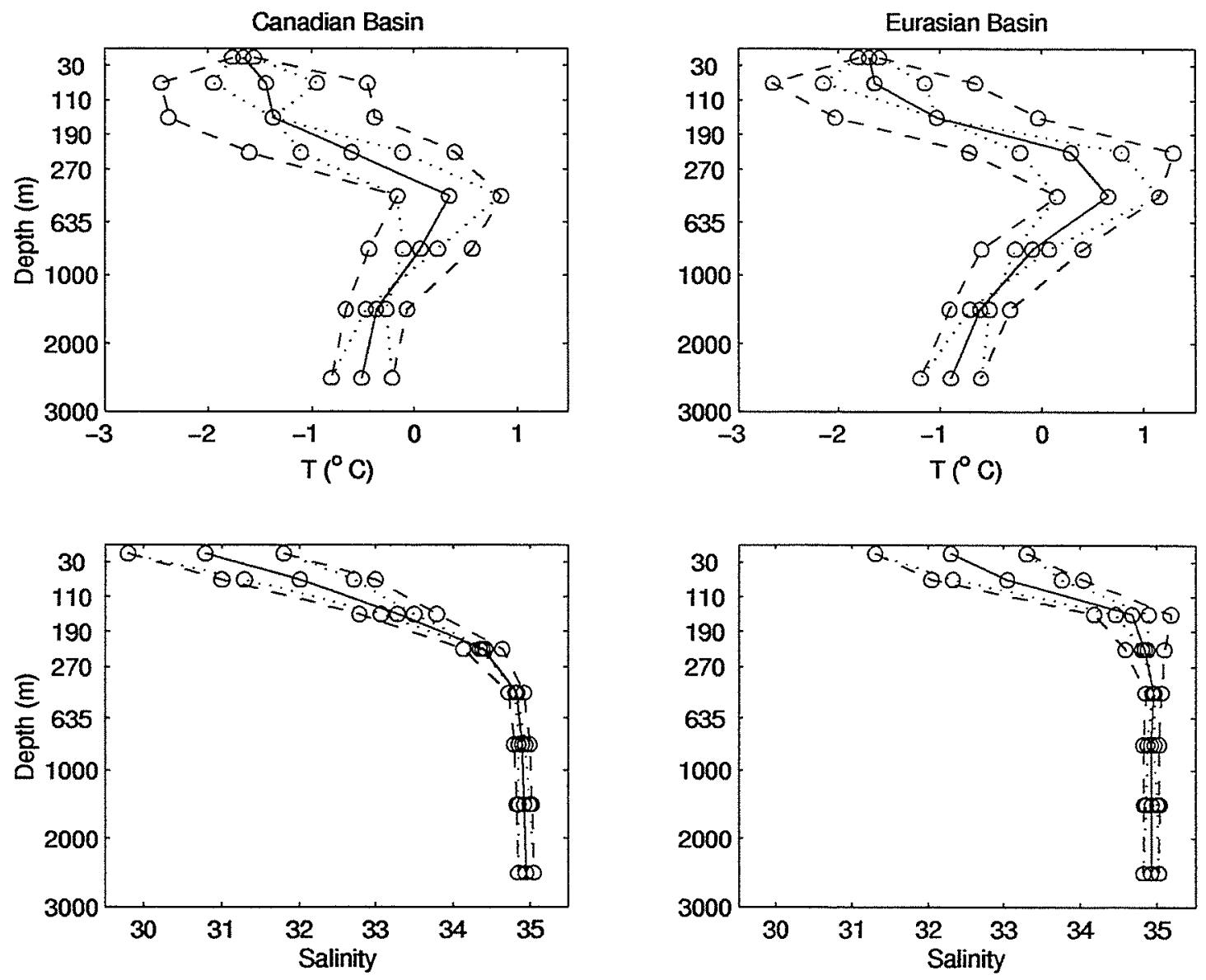

Figure 4.9: Limiting temperature and salinity profiles used in the sensitivity study. Solid lines: standard profiles. Dashed lines: minimum and maximum property values. Dotted lines: minimum and maximum vertical gradients. 
with a confidence interval of 1.2-4.6 Sv, as in Figure 4.4.) Based on this rough technique, then, accounting for the uncertainty in the basin $\mathrm{T} / \mathrm{S}$ profiles decidedly increases the uncertainty in the dense water formation rate, but not by as much as an order of magnitude.

The surface mixed layer exchanges were for the most part smaller in the sensitivity calculations than in the central calculation, primarily because the alternative profiles had larger differences between the surface box salinities, allowing the required relative salt flux to occur with lower transports.

\section{Shelf $\mathbf{T} / \mathrm{S}$}

Shelf temperature and salinity were varied by $\pm 1^{\circ} \mathrm{C}$ or psu. There was relatively little effect on the amount of dense water formation, except for the possibly counterintuitive result that the Eurasian Basin shelves formed more deep water when their salinity was lowered, and less when it was raised. The reason for this was that lower shelf salinity meant that the relative salt loading by the Barents Sea inflow was comparatively greater, requiring greater output of saline water to balance it. As was the case with the basin T/S study, surface mixed layer exchanges became less uncertain when changes in the shelf salinity increased the difference between the salinity values of the surface boxes.

\subsection{Estimates from Temperature, Salinity, and $\delta^{18} \mathbf{O}$}

To mitigate the uncertainty in the surface circulation, $\delta^{18} \mathrm{O}$ was added as a tracer, as described in Section 2.4. The $\delta^{18} \mathrm{O}$ conservation statements brought the total number of equations to 88 , with the same 83 unknowns. The rank of the system was 82 , and the solution nullspace was again eliminated by the non-negative constraints. 


\subsubsection{Optimal solution}

Recall from Section 4.2 that without $\delta^{18} \mathrm{O}$ constraints, the optimal solution included $5 \mathrm{~Sv}$ of surface mixed layer exchange in the Canadian Basin and almost $1 \mathrm{~Sv}$ of surface flow from the Eurasian Basin to the shelves. Addition of the $\delta^{18} \mathrm{O}$ constraints principally affected the solution by limiting the surface mixed layer exchange to a trickle of $O(0.1) \mathrm{Sv}$ from shelves to basin (Figure 4.10). Further, the diffusion between the surface mixed layers of the two basins was replaced by a 0.1-Sv "transpolar drift" from the Canadian Basin to the Eurasian. The $\delta^{18} \mathrm{O}$ constraints also shifted the distribution of waters produced on the Eurasian Basin shelf toward denser water. This water carried low- $\delta^{18} \mathrm{O}$ water to the deep basin, helping to maintain the $\delta^{18} \mathrm{O}$ minimum observed there, consistent with the conclusions of Bauch et al. (1995). The residuals in the $\delta^{18} \mathrm{O}$ inversion were somewhat larger than those in the calculations without $\delta^{18} \mathrm{O}$. However the solution was still consistent with the constraints within error bars (Figure 4.11). ${ }^{2}$

\subsubsection{Uncertainty of optimal solution}

Comparison of the Monte Carlo flux distributions (Figure 4.12) to those of the non-

\footnotetext{
${ }^{2}$ To ensure the independence of the constraints, the equations were rotated according to the prior estimate of the error covariance (Section 2.7.1), making physical interpretation of individual residuals difficult. Recall from Chapter 3 that errors in the the aggregate estimates (Section 3.5.1) for a given passage or flux are considered to be independent of the estimate errors in all the other passages, e.g., errors in the Bering Strait transport estimate are not correlated to those in the Arctic Archipelago transport estimate. However, in subsequent budgets (Sections 3.5.2, 3.5.3), such errors are correlated, through global equations such as the statement that the net transport into the Arctic must be zero. These flux error correlations then link the various constraints of the system: the mass conservation equation for box 2, of which the Bering input is a term, now contains errors which covary with the Arctic Archipelago errors appearing in the mass conservation equation for box 9. Mathematically, these appear as off-diagonal entries in the error variance-covariance matrix. When the system is scaled by this matrix, these off-diagonal terms rotate the equations, so the terms in a given equation no longer represent particular physical fluxes. Rather, the equation becomes a linear combination of several different physical constraints, and it is difficult to interpret the residual in terms of an individual constraint. It is possible to rotate the residuals back to "physical space," but doing so did not add a great deal of insight.
} 


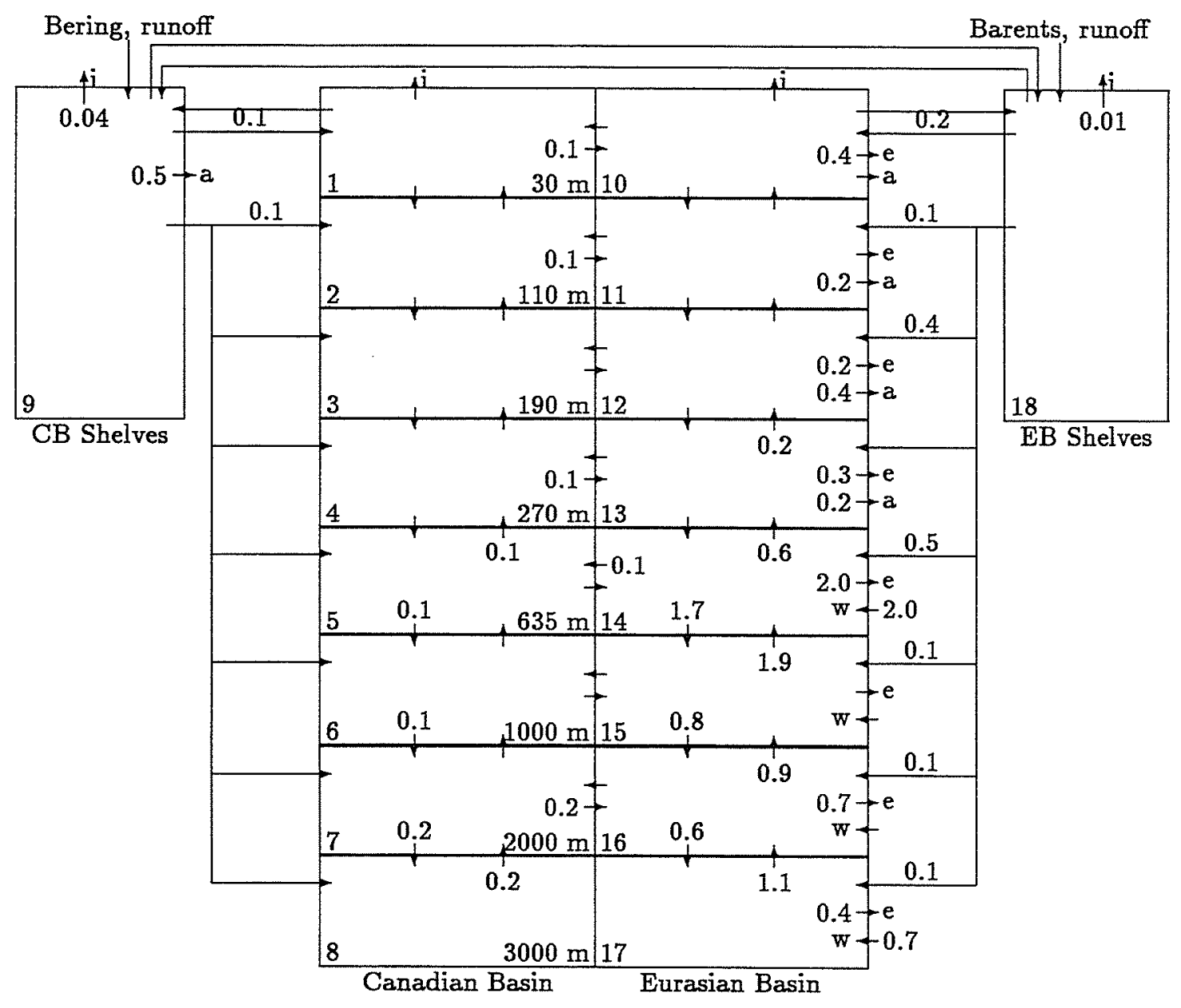

Figure 4.10: Solution of minimum residual norm (i.e., "steadiest" solution) for the temperature-salinity- $\delta^{18} \mathrm{O}$ inversion. Transports in Sverdrups are labeled as in Figure 4.1. 


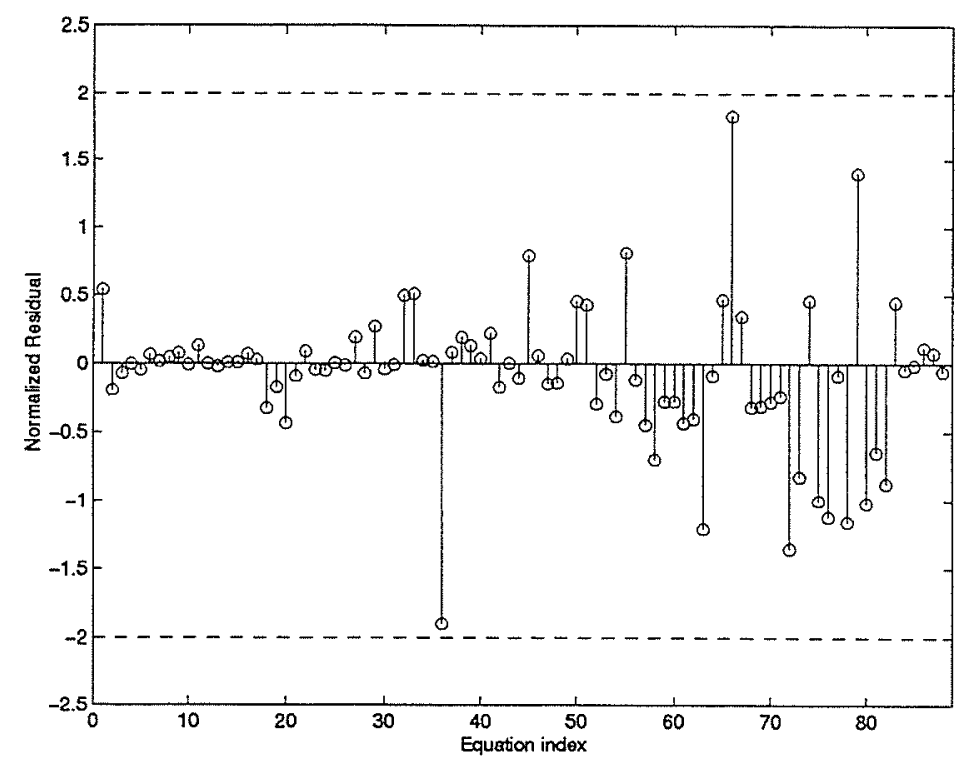

Figure 4.11: Equation residuals for the optimal solution of the temperaturesalinity $-\delta^{18} \mathrm{O}$ inversion, each normalized by the standard error of the equation.

$\delta^{18} \mathrm{O}$ solution (Figure 4.3) confirms the differences evinced in the optimal solution. The $\delta^{18} \mathrm{O}$ deficiency in the deep Eurasian Basin indeed required dense shelf water for its maintenance. Accordingly, some of the water previously directed to the halocline was diverted to depth. The $\delta^{18} \mathrm{O}$ constraints slightly lowered the amount of deep water formed in the Canadian basin.

The $\delta^{18} \mathrm{O}$ constraints significantly reduced the uncertainty of the deep water formation estimates in both basins (Figure 4.13). With the $\delta^{18} \mathrm{O}$ constraints, dense water formation was about $0.2 \pm 0.1 \mathrm{~Sv}$ in the Canadian Basin and about $1.4 \pm 0.3 \mathrm{~Sv}$ in the Eurasian Basin.

The decrease in uncertainty of the SML exchange was even more dramatic. The transports in each direction across the shelf break can be decomposed into mixing (equal and opposite exchange) and advection (the uncompensated part of the larger transport). Table 4.2 shows that the $\delta^{18} \mathrm{O}$ constraints virtually eliminated the mixing between the shelves and basins, which without $\delta^{18} \mathrm{O}$ was as high as $24 \mathrm{~Sv}$ 


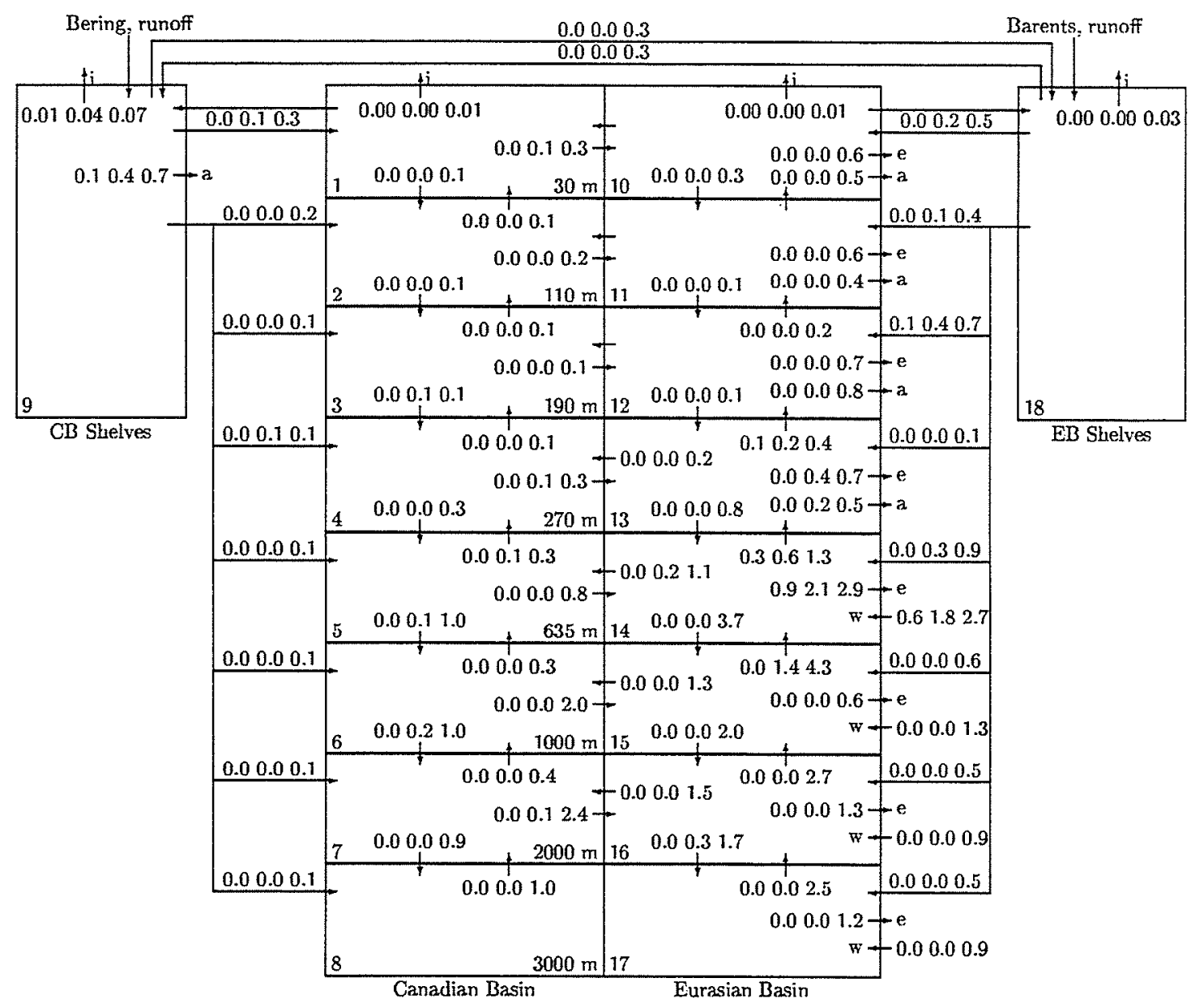

Figure 4.12: Monte Carlo transport distributions for each flux in the temperaturesalinity $-\delta^{18} \mathrm{O}$ inversion. Transports are presented as in Figure 4.3.

Table 4.2: Shelf-basin surface mixed layer exchange, with and without $\delta^{18} \mathrm{O}$ constraints. Advection is considered positive from shelf to basin.

\begin{tabular}{llccccccc}
\hline & & \multicolumn{3}{c}{ No $\delta^{18} \mathrm{O}$} & \multicolumn{3}{c}{ With $\delta^{18} \mathrm{O}$} \\
& & low & mode & high & low & mode & high \\
\hline $\mathrm{CB}$ & Mixing & 0 & 0 & 24.0 & 0 & 0 & 0.02 \\
& Advection & -3.0 & 0.1 & 0.6 & 0.03 & 0.10 & 0.30 \\
& & & & & & & \\
EB & Mixing & 0 & 0 & 1.4 & 0 & 0 & 0 \\
& Advection & -2.0 & 0 & 2.5 & 0 & 0.2 & 0.5 \\
\hline
\end{tabular}



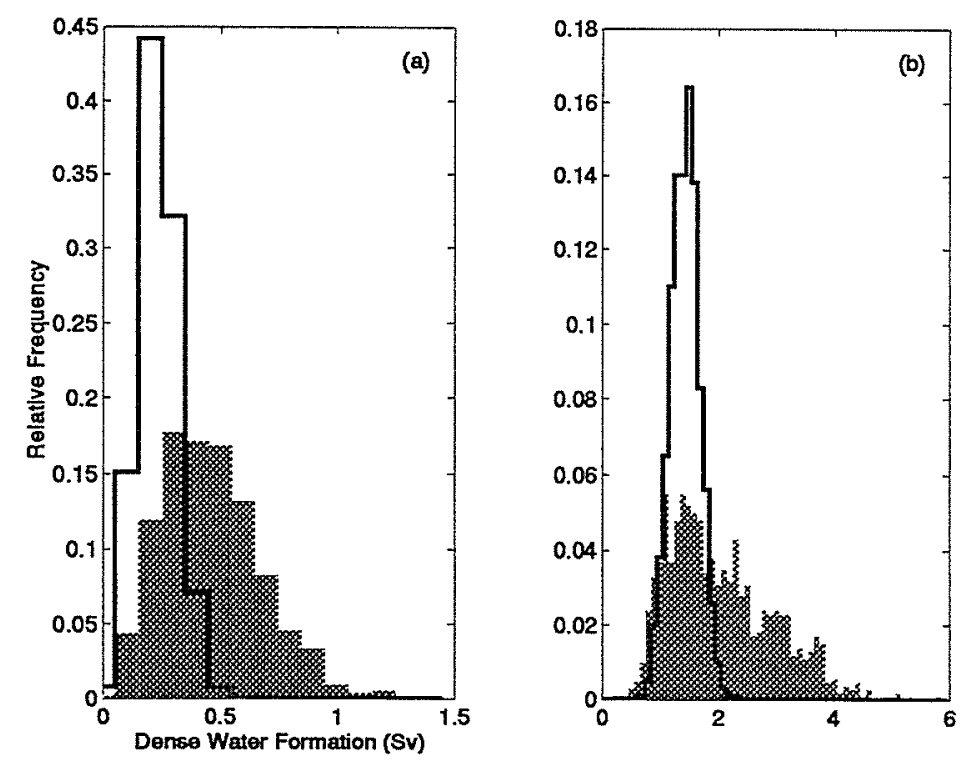

Figure 4.13: Histogram of 1000 Monte Carlo realizations of total dense water production with $\delta^{18} \mathrm{O}$ constraints (black line) and without (grey bars, repeated from Figure 4.5), for (a) the Canadian Basin, and (b) the Eurasian Basin. Bin size in both panels is $0.1 \mathrm{~Sv}$, but the plot scales differ.

in the Canadian Basin. With $\delta^{18} \mathrm{O}$, the advective flux was confined to a few tenths of a Sverdrup from shelves to basins, where without it, even the sign was uncertain.

The distributions of the residuals from the $\delta^{18} \mathrm{O}$-conserving calculations showed that over the full range of plausible data values, the solutions met all constraints (Figure 4.14).

\subsubsection{Extreme solutions}

Linear programming solutions further confirmed that $\delta^{18} \mathrm{O}$ conservation considerably restricted the range of shelf-basin exchange permitted in the model. With $\delta^{18} \mathrm{O}$, the minimum dense water formation was $0.8 \mathrm{~Sv}$, the maximum, $3.1 \mathrm{~Sv}$; without $\delta^{18} \mathrm{O}$, the extremes had been 0 and $9.4 \mathrm{~Sv}$ (Table 4.3). In the minimum case, $0.1 \mathrm{~Sv}$ were directed to the deep Canadian basin and the rest went to the lower 


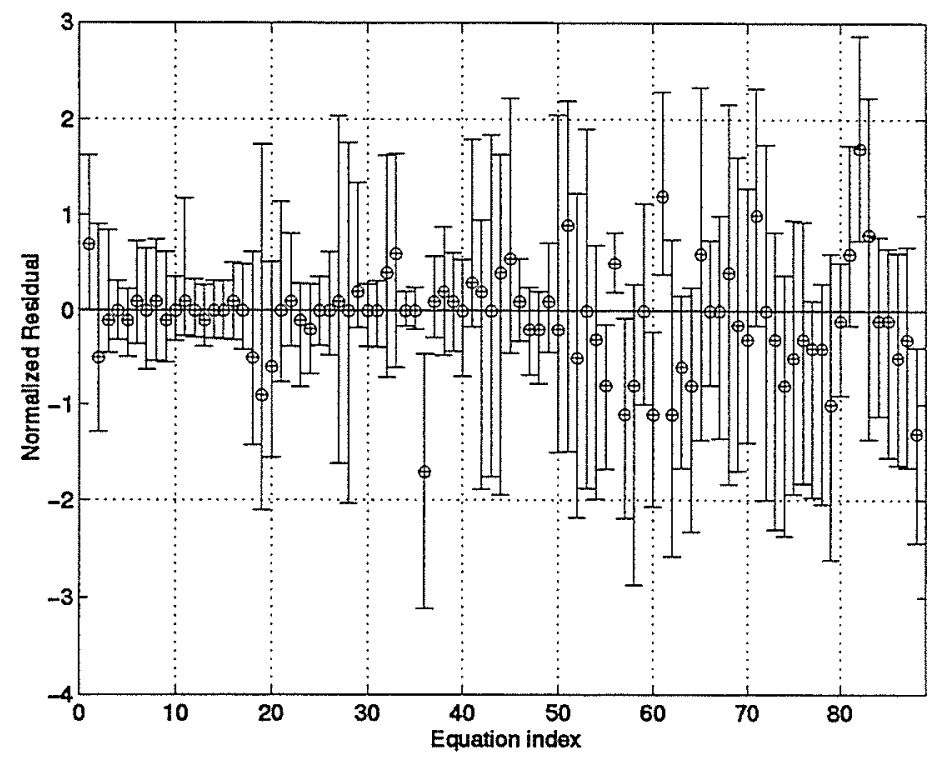

Figure 4.14: Monte Carlo modes (o) and 95\% intervals of the residuals for the temperature-salinity- $\delta^{18} \mathrm{O}$ inversion.

halocline and upper Atlantic layer of the Eurasian basin. In the maximum case, $0.7 \mathrm{~Sv}$ were distributed evenly over the Canadian Basin boxes, and the remaining $2.4 \mathrm{~Sv}$ were distributed almost evenly among the Eurasian Basin boxes. The range of surface mixed layer exchange was even more greatly reduced. The minimum exchange was zero. The maximum included $0.3 \mathrm{~Sv}$ advection from the shelves to the Canadian Basin, and 0.7 Sv in each direction between the Eurasian Basin and

Table 4.3: Extreme totals of shelf-basin exchange in Sverdrups, with and without $\delta^{18} \mathrm{O}$ constraints.

\begin{tabular}{clcc}
\hline & & Min & Max \\
\hline Dense Water Flux & no $\delta^{18} \mathrm{O}$ & 0.0 & 9.4 \\
& with $\delta^{18} \mathrm{O}$ & 0.8 & 3.1 \\
& & & \\
SML Exchange & no $\delta^{18} \mathrm{O}$ & 0.0 & 72.2 \\
& with $\delta^{18} \mathrm{O}$ & 0.0 & 1.7 \\
\hline
\end{tabular}


the adjacent shelves, far less than the $72 \mathrm{~Sv}$ of total surface mixed layer flux which had been permitted in the absence of $\delta^{18} \mathrm{O}$ constraints.

Overall, the $\delta^{18} \mathrm{O}$ conservation requirements established that at least $0.8 \mathrm{~Sv}$ of dense water formation is required for steady state, most but not all of it in the Eurasian Basin. It is likely that on average there is weak advection in the surface mixed layer from the shelves to the basins, and from the Canadian Basin to the Eurasian. No flux is required from the basin surface mixed layers to the shelves.

\subsubsection{Sensitivity to prescribed $\delta^{18} \mathrm{O}$}

Since the prescribed values of $\delta^{18} \mathrm{O}$ used in these studies were based on sparse data, it was important to determine whether revisions would have a substantial effect on the results. Two calculations were performed, in which the basin $\delta^{18} \mathrm{O}$ values were respectively lower and higher than in the standard case (Figure 2.3). Lowering the $\delta^{18} \mathrm{O}$ values in the basin made very little difference to the solution, and only slightly raised one or two of the residuals. Increasing the $\delta^{18} \mathrm{O}$ increased the dense water flowing to the Eurasian basin halocline, from $0.6 \pm 0.2 \mathrm{~Sv}$ to approximately $1.1 \pm 0.3 \mathrm{~Sv}$. Shelf-basin mixing in the SML became more uncertain, with zero modes in both basins as before, but with $95 \%$ intervals ranging up to $2.2 \mathrm{~Sv}$ in the Canadian Basin and 0.5 Sv in the Eurasian. The higher basin $\delta^{18} \mathrm{O}$ also shifted the mode of Eurasian Basin shelf-SML advection to zero, with a $95 \%$ interval of $-0.3-0.3 \mathrm{~Sv}$. The residuals were not affected by the higher $\delta^{18} \mathrm{O}$ values.

The prescribed values of shelf $\delta^{18} \mathrm{O}(-1.9 \%$ for the Canadian Basin shelves, $-0.1 \%$ for the Eurasian Basin shelves) were even more uncertain than the basin values, and a different approach was taken to exploring their effect. The shelf values of $\delta^{18} \mathrm{O}$ were varied from $2 \%$ below the standard values, up to $0.3 \%$, the highest value observed in the Arctic. The volume of dense water formed as a function of the perturbation from the standard values is plotted in Figure 4.15. 


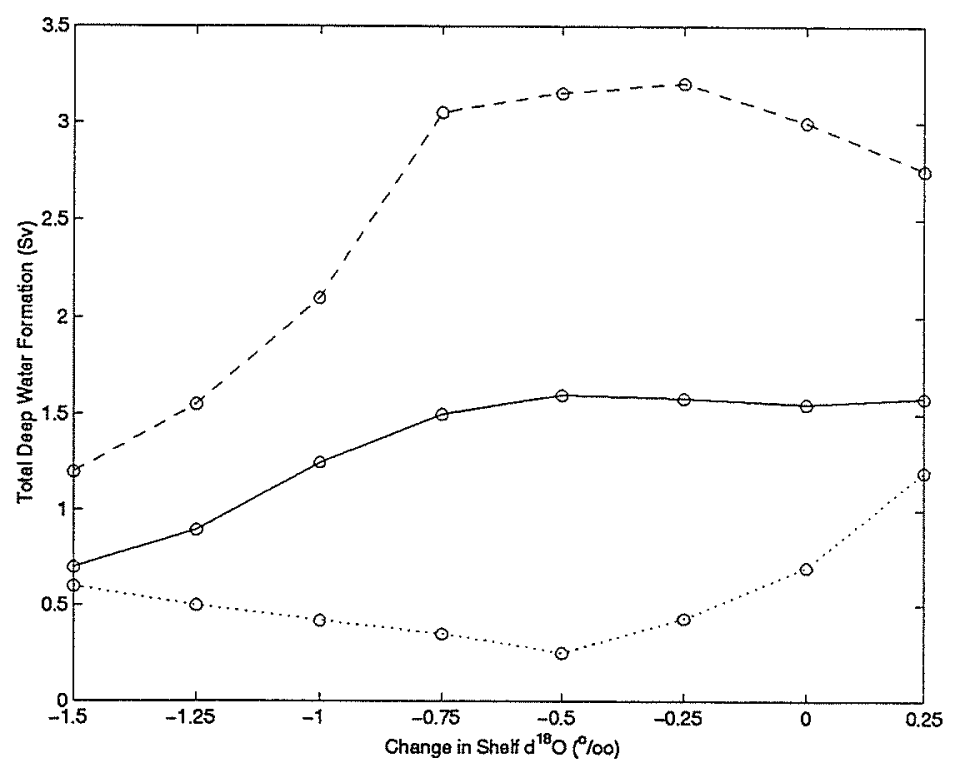

Figure 4.15: Minimum (dotted curve), maximum (dashed), and optimal (solid) dense water formation vs. perturbation to standard shelf $\delta^{18} \mathrm{O}$ values $(-1.9 \%$ for the Canadian Basin shelves, $-0.1 \%$ for the Eurasian Basin). Larger perturbations precluded consistent solutions.

When the perturbation is between -1.50 and $0.25 \%$, the range delineated by the extreme values varies from $0.5 \mathrm{~Sv}$ to nearly $3 \mathrm{~Sv}$ wide. Even at its widest, though, the range is much narrower than without the $\delta^{18} \mathrm{O}$ constraints. The optimal solution varies by less than a Sverdrup, and the residuals of the optimal solution (not shown) are not unacceptably large. For perturbations outside this range, however, there is a qualitative change: no solution is capable of meeting the linear programming constraints, and the optimal solution suffers large residuals. In other words, consistent, steady solutions do not exist if the shelf values of $\delta^{18} \mathrm{O}$ are outside the range of $-1.50 \%$ to $0.25 \%$. 


\subsection{Effects of the Model Assumptions}

In addition to the prescribed temperature and salinity and $\delta^{18} \mathrm{O}$ profiles discussed above (Sections 4.2.4 and 4.3.4), several other assumptions of the model design influenced the results. Among these were the pathways of runoff, the Arctic Archipelago outflow, and the West Spitzbergen Current, the omission of ice melt or cross-shelfbreak upwelling, the size and sign of surface heat flux in the basin, and the error estimates ascribed to the conservation statements and to the forcing data. The effects of these factors are now discussed.

\subsubsection{Representation of river runoff}

The model faces a limitation concerning the maintenance of the $\delta^{18} \mathrm{O}$ minima in the basin surface boxes. River runoff is mixed into the shelves before flowing to the basins, and the resulting mixture is isotopically heavier than the basin surface layers. Therefore there is no source of "light" (low- $\left.\delta^{18} \mathrm{O}\right)$ water which can maintain the basin surface $\delta^{18} \mathrm{O}$ minima. To satisfy $\delta^{18} \mathrm{O}$ conservation in the basin, then, the solution must minimize the mixing between the basin surface mixed layers and other boxes.

This is somewhat concerning, because the existence of the observed $\delta^{18} \mathrm{O}$ minima in the basins implies that at least some runoff must cross the shelves relatively coherently, supplying low- $\delta^{18} \mathrm{O}$ water to the basins (Bauch, 1995; see also Section 6.2 for further discussion). The absence of this mechanism from the model is therefore unrealistic. ${ }^{3}$ The question is, is the unnatural absence of a low $-\delta^{18} \mathrm{O}$ source for the basins the main cause of the low solution uncertainty? If so, the $\delta^{18} \mathrm{O}$-conserving calculations would have to be dismissed as irrelevant.

\footnotetext{
${ }^{3}$ Worse, it may be backwards: assuming that in truth, some river runoff makes its way to the basin before mixing completely with the shelves, one would assume that increased runoff would tend to lighten (isotopically) the basins. In the present model, though, increased runoff might cause more flow of the relatively heavy shelf water to the basins-the opposite effect! (J. Marotzke, personal communication, 1998)
} 
To find out if this was the case, calculations were made in which part of the river runoff was diverted directly to the basins. Starting with zero diversion (the standard case), the fraction of runoff which entered the basins directly was increased $10 \%$ at a time until all runoff bypassed the shelves entirely. When runoff was directed to the basins, the maximum amount of consistent shelf-basin surface exchange doubled to approximately $5 \mathrm{~Sv}$. Other than this, there were no significant changes in the shelf-basin exchange. Further, once some runoff was diverted directly to the shelves, the amount didn't seem to matter (Figure 4.16). Apparently, the restrictions on surface layer mixing imposed by the $\delta^{18} \mathrm{O}$ constraints are "real", in that they are not caused by the absence of a low- $\delta^{18} \mathrm{O}$ source for the basins. This is discussed further in Section 6.2.

\subsubsection{Representation of the Arctic Archipelago}

To examine the influence of the Arctic Archipelago representation, the calculation of Section 4.3 was repeated exactly, except that instead of originating on the Canadian Basin shelves and in the Eurasian Basin, the Arctic Archipelago outflow was drawn solely from the top four boxes of the Canadian Basin (Figure 4.17). Only two differences resulted. First, the increased drainage of Canadian Basin halocline water required an additional $0.3 \mathrm{~Sv}$ of dense shelf water to replace it (Figure 4.18). The total dense water production increased by the same amount, i.e., the extra water sinking to the halocline was created additionally and not diverted from elsewhere. The Bering Strait mass input, which in the standard case was partially balanced by the Arctic Archipelago outflow, helped feed the additional dense wa-

ter flux. Second, the $\delta^{18} \mathrm{O}$ budget for the lowest $\mathrm{CB}$ halocline box was violated by 2-4 standard errors, owing to the increased volume of low- $\delta^{18} \mathrm{O}$ shelf water. This misfit should be interpreted with caution, considering how poorly $\delta^{18} \mathrm{O}$ is constrained in the Canadian Basin and especially on the shelves. 

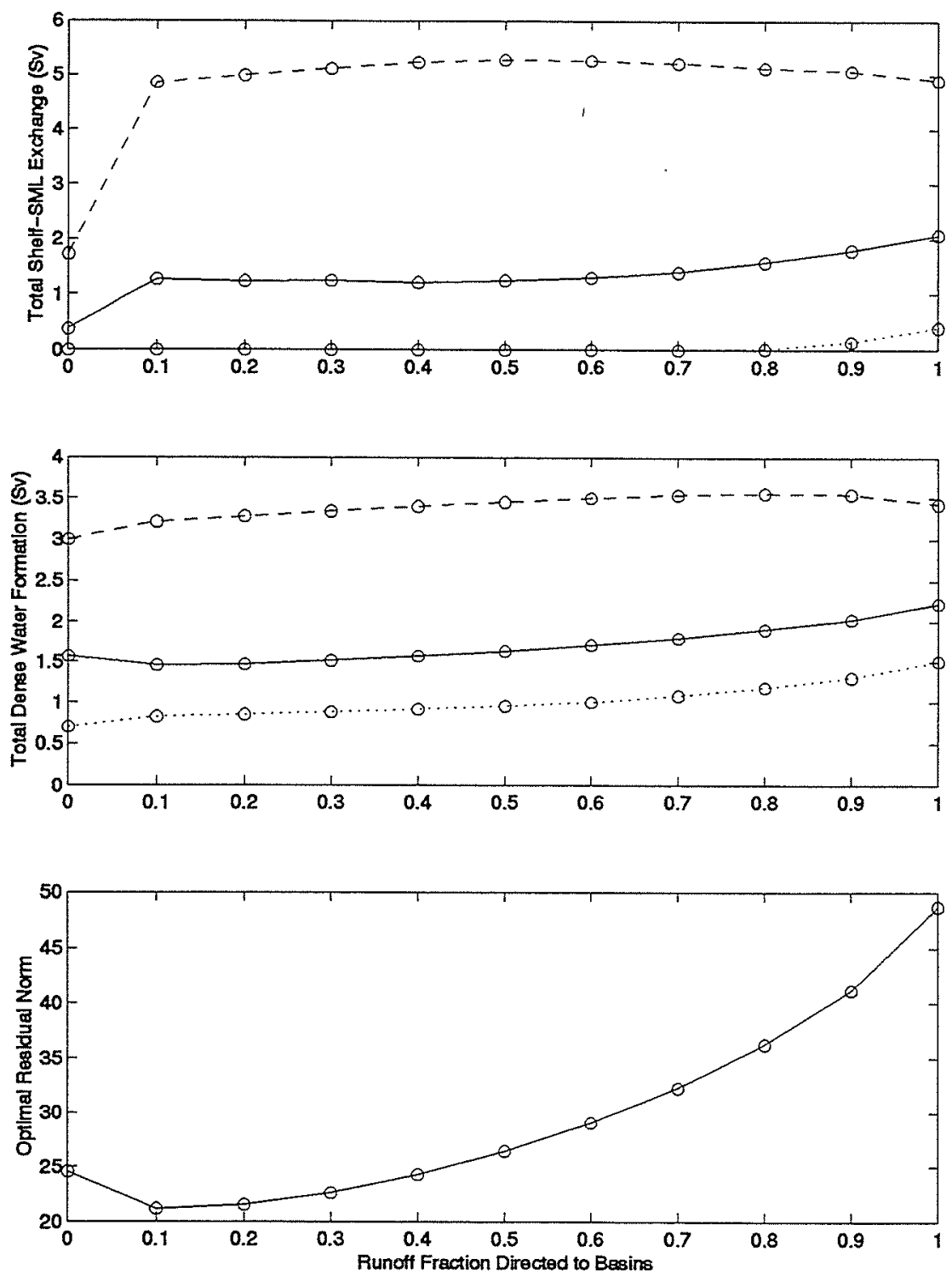

Figure 4.16: Shelf-basin exchange and solution residual norm vs. fraction of river runoff diverted directly to the basins. Zero diversion is the standard case. Top: maximum (dashed), minimum (dotted), and optimal (solid) total surface mixed layer exchange. Middle: maximum (dashed), minimum (dotted), and optimal (solid) total dense water formation. Bottom: norm of residual norm $\|\mathbf{n}\|$ for the optimal calculation. Note that even when $\|\mathbf{n}\|$ is at its highest, the solution satisfies all constraints within two standard errors. 


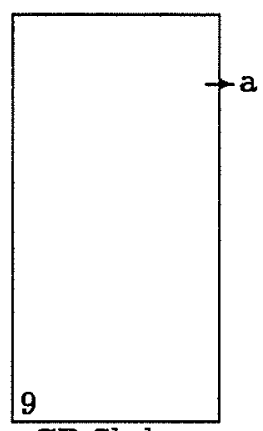

CB Shelves

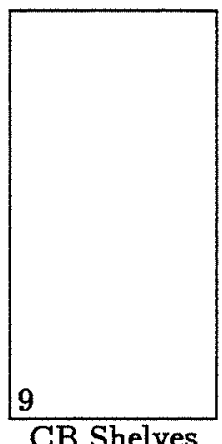

CB Shelves

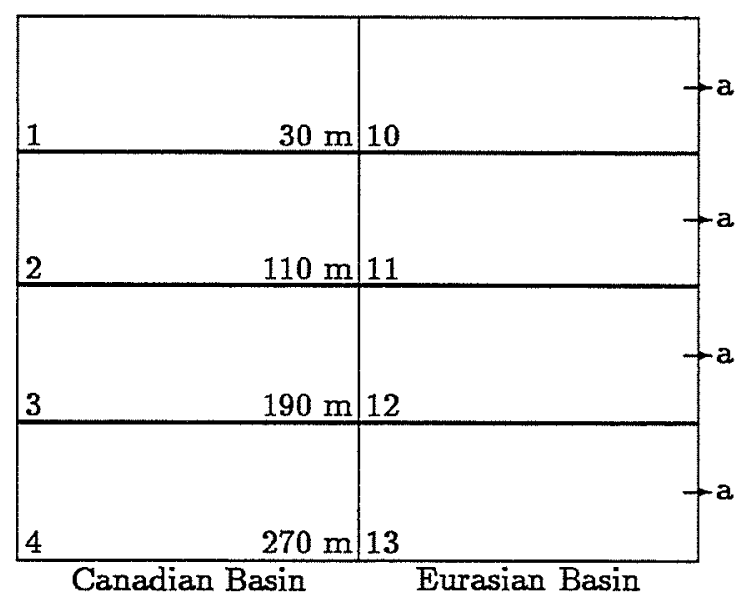

18

EB Shelves

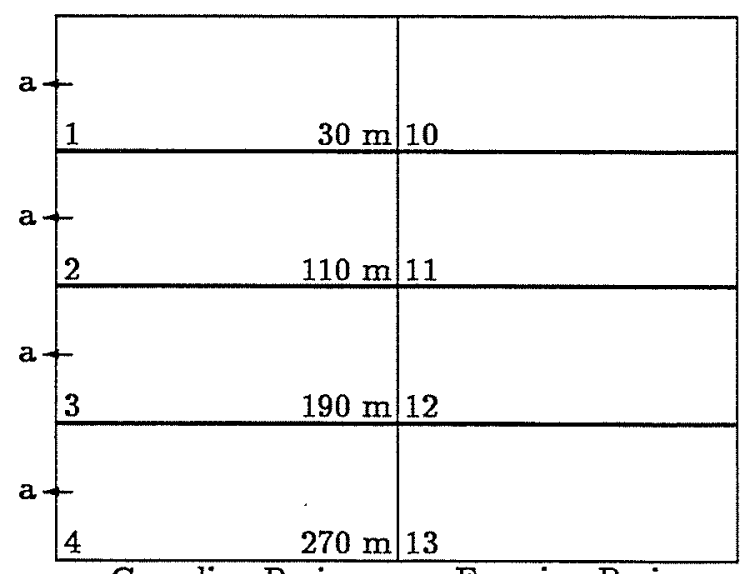

18

EB Shelves

Figure 4.17: Alternative sources for the Arctic Archipelago outflow. Upper: the standard assumption of this study, as described in Section 2.6.2. Outflow drawn from the Eurasian Basin through the Nares Strait, and from the Canadian Basin shelves through Jones and Lancaster Sound. Lower: the alternative tested in this section. Outflow drawn entirely from the Canadian Basin. 


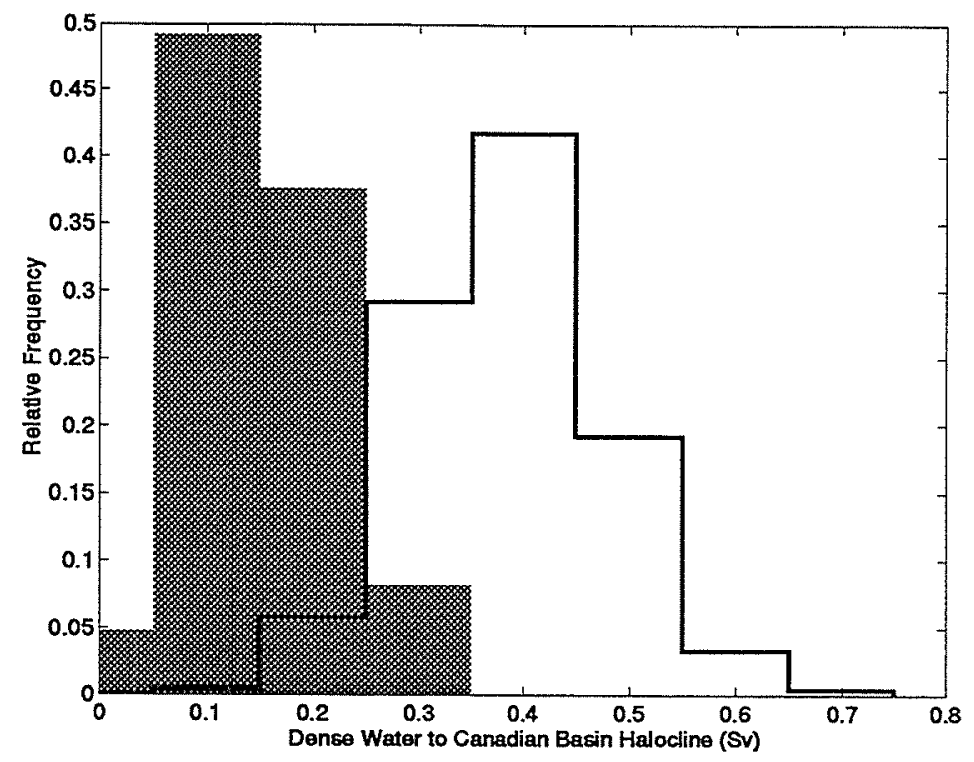

Figure 4.18: Histogram of 1000 Monte Carlo realizations of transport of dense water from Canadian Basin shelves to the halocline in the temperature-salinity$\delta^{18} \mathrm{O}$ inversion. Grey bars: Arctic Archipelago drawn from the Canadian Basin shelves and from the Eurasian Basin, as described in Section 2.6.2. Black line: Arctic Archipelago drawn entirely from the upper Canadian Basin.

\subsubsection{Representation of the West Spitzbergen Current}

Rudels et al. (1996) have suggested that the denser waters of the West Spitzbergen Current directly enter the Eurasian Basin halocline, where they are mixed into the halocline water, instead of diving under the Polar Water as has been assumed and parameterized here and previously. To explore the viability of their scenario, a calculation was performed in which the WSC was allowed to enter the top six boxes of the Eurasian Basin (the SML, the halocline, and the Atlantic Layer), instead of just the fifth and sixth boxes. The total inflow was constrained as described in Section 2.6.3.

Given the freedom to do so, almost half the incoming Atlantic Water entered the lower halocline (box 13) instead of the Atlantic layer (boxes 14 and 15). About 

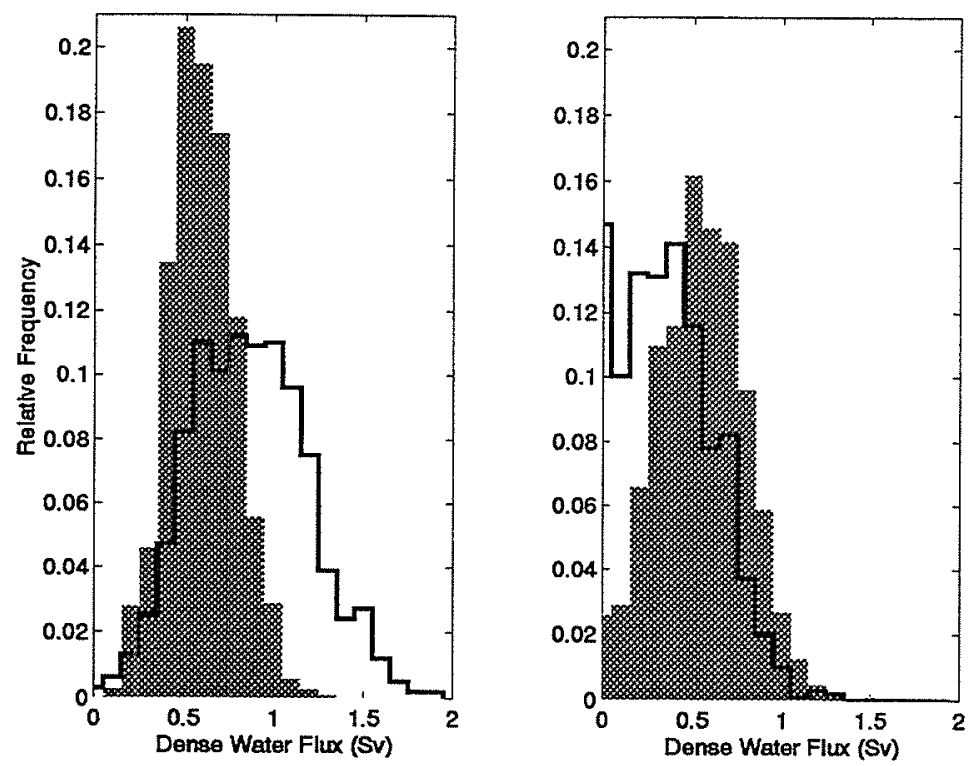

Figure 4.19: Histogram of 1000 Monte Carlo realizations of transport of dense water from Eurasian Basin shelves to the halocline (left) and the Atlantic Layer (right) in the Transfer Shelf model with $\delta^{18} \mathrm{O}$. Black line: West Spitzbergen Current directed to upper six boxes of Eurasian Basin. Grey bars: West Spitzbergen Current directed only to Atlantic Layer boxes.

$0.3 \mathrm{~Sv}$ of the dense shelf water which had flowed to the Atlantic layer in previous calculations was diverted to the lower halocline (Figure 4.19). Residuals were smaller than in other calculations, but not significantly. There were no other differences between the solutions. Thus, solutions in which the Atlantic water could enter the halocline directly, and solutions in which it could only enter through the Atlantic layer, were equally consistent with the constraints of data and conservation. In other words, the data are not sufficiently powerful to distinguish between the scheme of Rudels et al. and the traditionally assumed "diving" West Spitzbergen Current. However, the solutions do show that in steady state, the Rudels et al. West Spitzbergen Current scheme implies a lower average density for water formed on the Eurasian shelves. 


\subsubsection{Representation of diffusion}

To this point diffusion has been a model variable, appearing in the solutions as equal and opposite transports between adjacent boxes, and only as required to meet the model constraints. This representation has allowed unforced regions of the model to remain isolated. For example, the deep salinity maximum of the Canadian Basin has been maintained in the solutions simply by not disturbing it very much. Though this is not entirely unrealistic, as residence times are thought to be much longer in the Canadian Basin than in the Eurasian Basin (Östlund et al., 1987), it is worthwhile to determine if the model could maintain such features against an enforced minimum diffusion.

Because the nature, strength, and spatial distribution of mixing in the Arctic are unknown, these questions were explored using only the crudest representation of diffusion, namely, an enforced minimum reciprocal transport between each box and its neighbors. Vertical exchanges were set to $1 / 10$ the strength of horizontal exchanges. No error was ascribed to this additional forcing.

As the diffusive forcing was varied from zero to $0.06 \mathrm{~Sv}$ (horizontal), there were no significant changes in the amount of shelf-basin exchange except for a decrease in the maximum consistent value of surface mixed layer exchange (Figure 4.20). Greater diffusion, however, precluded consistent solutions, mainly due to the model's inability to maintain the $\delta^{18} \mathrm{O}$ minimum in the Canadian Basin surface mixed layer. The optimal value of dense water flux to the bottom of the Canadian Basin did increase, in order to preserve the deep salinity maximum there, but the increase was from a minimum of $0.0175 \mathrm{~Sv}$ at zero diffusion to a maximum of $0.0235 \mathrm{~Sv}$ when horizontal diffusion was set to $0.06 \mathrm{~Sv}$, i.e., no change in the first significant figure. 

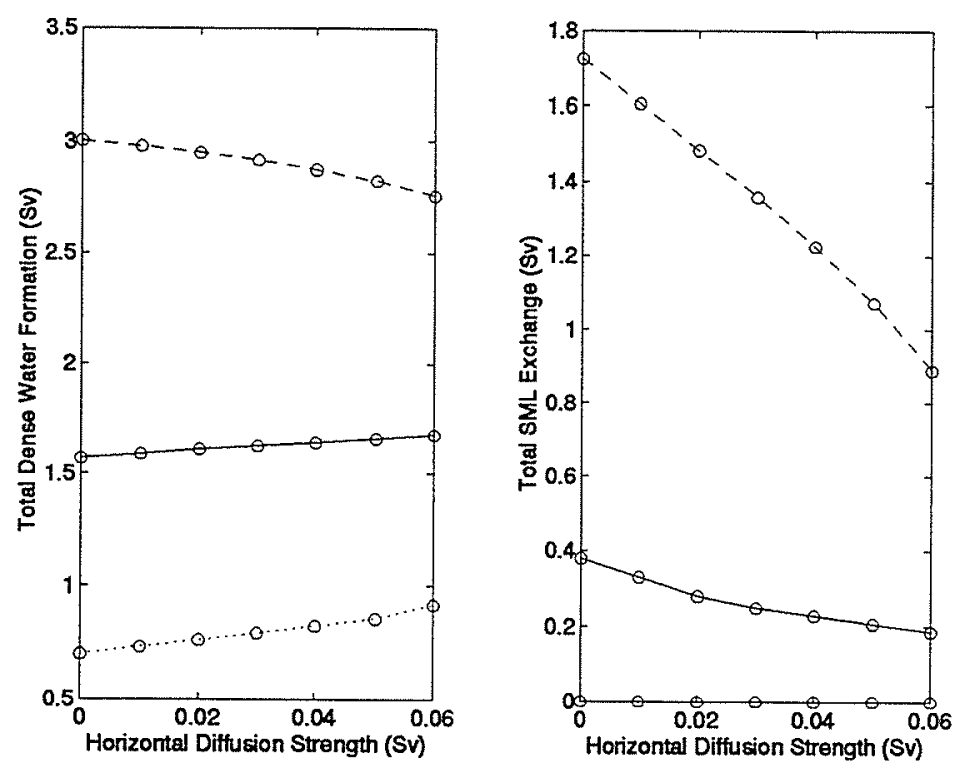

Figure 4.20: Dense water formation (left) and shelf-basin surface mixed layer exchange (right) vs. prescribed minimum horizontal circulation rate. Minimum vertical circulation was $1 / 10$ the horizontal rate. No feasible solution was possible for horizontal rates greater than $0.06 \mathrm{~Sv}$. Minimum (dotted curve), maximum (dashed), and optimal (solid) shelf-basin exchange rates are shown.

\subsubsection{Inclusion of cross-shelfbreak upwelling}

The importance of upwelling across the shelfbreak has been a persistent but elusive topic of speculation in the Arctic literature (Aagaard et al. 1981; Aagaard and Roach, 1990; Grebmeier et al. 1998). The model results described above have shown that to within the resolution of the available data, shelfbreak upwelling is not required to maintain steady state in the Arctic. It remains to determine the maximum amount of upwelling consistent with steady state, and whether the presence of upwelling significantly changes the possible range of shelf-basin exchange. Adding upwelling introduces new freedom to the model without adding new constraints, so solutions with smaller residuals and greater uncertainty are expected. However, the amount of upwelling is likely to be limited: the upwelled halocline 
Table 4.4: Minimum and maximum consistent amounts of dense water formation, surface mixed layer exchange, and shelfbreak upwelling in Sverdrups for the temperature-salinity- $\delta^{18} \mathrm{O}$ inversion.

\begin{tabular}{lcc}
\hline & $\begin{array}{c}\text { no } \\
\text { shelfbreak } \\
\text { upwelling }\end{array}$ & $\begin{array}{c}\text { with } \\
\text { shelfbreak } \\
\text { upwelling }\end{array}$ \\
\hline dense water formation & $0.8-3.1$ & $0.7-4.6$ \\
surface mixed layer exchange & $0.0-1.7$ & $0.0-2.1$ \\
shelfbreak upwelling & & $0.0-3.8$ \\
\hline
\end{tabular}

water must be replaced, which draws on the rest of the system (see Section 5.3 below).

To investigate the role of shelfbreak upwelling, a calculation identical to the temperature-salinity- $\delta^{18} \mathrm{O}$ inversion of Section 4.3 was performed, with an additional pipe in each basin allowing flux to the shelves from the deepest halocline box (190-270 m). The optimal solution included no upwelling in the Canadian Basin, and 0.3 Sv of upwelling in the Eurasian Basin. The Eurasian Basin upwelling allowed an additional $0.3 \mathrm{~Sv}$ of dense water production, distributed over the entire range of densities. The residual norm was only incrementally lower than it was without upwelling (4.92 vs. 4.96). The maximum consistent amount of shelfbreak upwelling, determined by linear programming, was $3.8 \mathrm{~Sv}$ (Table 4.4); in other words, it was restricted to a maximum value as expected. Further linear programming calculations showed that shelfbreak upwelling endowed the model with some freedom, but not a great deal: the maximum consistent amount of dense water formation grew to $4.6 \mathrm{~Sv}$, the maximum surface mixed layer exchange grew to $2.1 \mathrm{~Sv}$ (Table 4.4 ). 


\subsubsection{Inclusion of ice melt and advection}

Ice melt and ice advection were not represented in the standard runs, and it was desirable to determine how their presence might affect the solution. To this end, four ice boxes were added to the model, one above each surface box. Each had the ability to take up water from the surface mixed layer, representing ice formation, or return it, representing ice melt. Flow was also allowed between adjacent ice boxes, representing ice advection. Volume was conserved in each ice box; heat and salt were not explicitly conserved since those constraints would have been nearly exact multiples of the volume constraint. Conservation of $\delta^{18} \mathrm{O}$ in each ice box was optional: without it, the model system had 91 constraints on 95 unknowns, with rank 85; with $\delta^{18} \mathrm{O}$ conservation, there were 95 constraints on 95 unknowns, with rank 88. The non-negative constraints eliminated the solution nullspace.

Fluxes representing ice formation carried the $\delta^{18} \mathrm{O}$, temperature, and salinity values used above (Section 3.3.2). Fluxes from the ice boxes, i.e., ice melt and advection, carried the salinity and effective temperature of sea ice, and the $\delta^{18} \mathrm{O}$ of the originating box. This presented a problem: what $\delta^{18} \mathrm{O}$ to assign to the ice boxes? Following Bauch (1995), it was initially assumed that the average $\delta^{18} \mathrm{O}$ of each box was equal to that of locally formed ice, i.e., the local surface value plus the fractionation factor of $2.1 \%$. The NNLS solution was then calculated without $\delta^{18} \mathrm{O}$ conservation in the ice boxes, and the $\delta^{18} \mathrm{O}$ balance for the ice boxes was diagnosed a posteriori. Some advection of ice appeared in the solution, so in fact not all the ice in a given box was formed locally. However, the effect of this advection on the $\delta^{18} \mathrm{O}$ budget of each ice box was small: $\delta^{18} \mathrm{O}$ was conserved in every box to $0.07 \mathrm{~Sv} \cdot \%$ or better. Since the assumed values for ice $\delta^{18} \mathrm{O}$ were consistent with $\delta^{18} \mathrm{O}$ conservation in the ice boxes, the $\delta^{18} \mathrm{O}$ conservation constraints were used in subsequent calculations.

Linear programming was again used to determine the maximum and minimum 
values of shelf-basin exchange consistent with the constraints. The additional freedom of ice melt and advection did not significantly widen the ranges of consistent values: the only noticeable change was to lower the minimum consistent dense water formation rate from $0.8 \mathrm{~Sv}$ to $0.5 \mathrm{~Sv}$. Some Eurasian Basin ice melt of $O(0.01 \mathrm{~Sv})$ appeared in the solutions; this was accompanied by ice advection from the shelves to the basins of $0.01-0.05 \mathrm{~Sv}$.

By assimilating buoy tracks and other information into a simple ice model, Thomas et al. (1996) have estimated the net ice melt and the ice advection for the various regions of the Arctic. An attempt was made to further constrain the present model with the ice motions and melt estimated by Thomas et al., but without success. The mapping of their results onto the comparatively coarse resolution of the box model produced relatively large error bars on the ice constraints (Appendix B). As a result, the box model solutions were not greatly affected, nor did the model have any trouble finding solutions consistent with Thomas et al.'s estimates.

\subsubsection{Sensitivity to prescribed basin surface heat flux}

As was discussed in Section 3.3.3, estimates of the basin surface heat flux are tenuous at best. In the absence of $\delta^{18} \mathrm{O}$ constraints, uncertainty in the surface heat flux leads to wild uncertainty in the surface circulation (Sections 4.2 .2 and 4.2.3). The addition of $\delta^{18} \mathrm{O}$ went a long way toward taming the uncertainty, but the question still lingers of whether a different choice of basin surface heat flux would significantly alter the results.

The answer is no. This was confirmed by a series of calculations in which the surface heat flux was varied from -10 to $10 \mathrm{~W} \mathrm{~m}^{-2}$, with the uncertainty held constant at $\pm 5 \mathrm{~W} \mathrm{~m}^{-2}$. Over the entire range, the optimal and extreme values of shelf-basin exchange (not shown) were almost constant. Mass, salt, and 


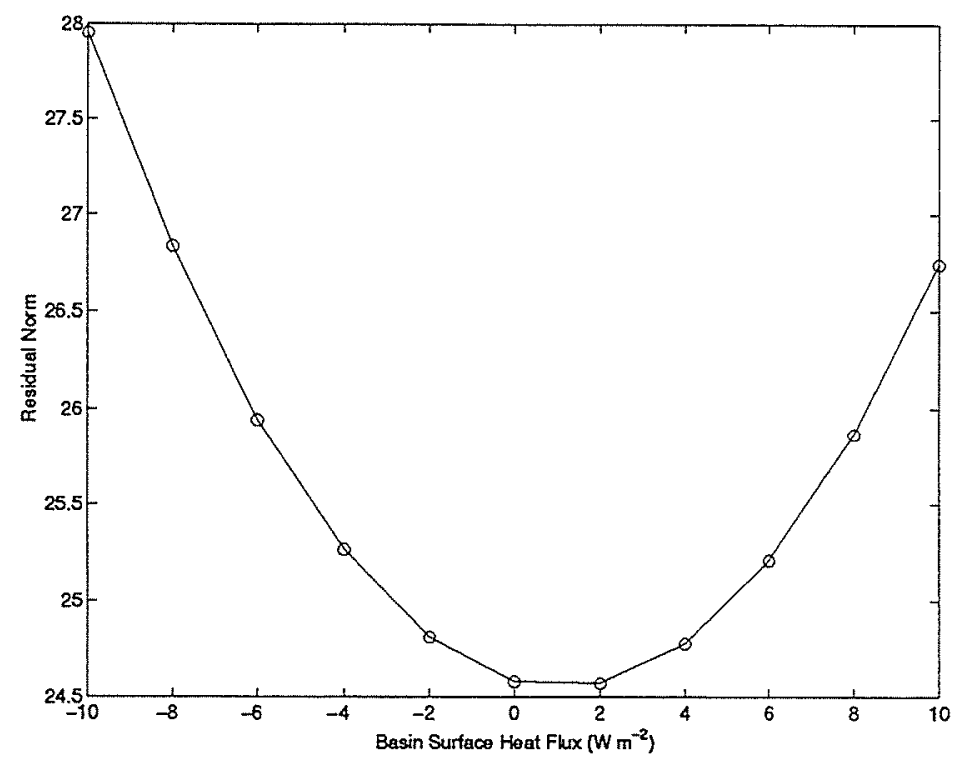

Figure 4.21: Residual norm $\|\mathbf{n}\|$ vs. prescribed basin surface heat flux. Even though the residual norm is higher in some cases than in others, solutions across the entire range of prescribed heat flux were consistent with the constraints.

especially $\delta^{18} \mathrm{O}$ conservation place constraints on the surface circulation and related dense water formation which are strict compared to the relatively uncertain heat budgets of the surface boxes. As a result, the model solution can be a long way from conserving heat at the surface, and still be consistent within error bars. In the presence of other, more demanding constraints, the surface heat budget is all but ignored, and so changes in the prescribed surface heat flux make no difference in the minimum, maximum, or optimal values of both dense water formation and surface heat flux.

As an aside, the residual norm was smallest when the heat flux was slightly positive, i.e., heat flux into the ocean (Figure 4.21). However, even when the surface heat flux was $-10 \mathrm{~W} \mathrm{~m}^{-2}$ and the residual norm was at its highest, all constraints were satisfied within two standard errors, and most were satisfied to within 1.5 standard errors. 


\subsubsection{Sensitivity to prescribed uncertainty}

Two kinds of uncertainties were prescribed in the model: the uncertainties of the conservation equations, and the uncertainties in the boundary forcing. This section explores the impact on the solutions of these choices.

Sensitivity to budget imbalance tolerance The prior estimates of uncertainty of the conservation equations (Section 2.5) were determined informally, and it was desirable to determine the sensitivity of the model results to their values. Therefore, calculations were repeated, with the standard conservation uncertainties multiplied by a factor ranging from 0.1 to 10.0 . The extreme (linear programming) and optimal (non-negative least squares) values of total dense water production are plotted in Figure 4.22 as a function of the multiplication factor. When the estimates were reduced to $1 / 4$ of their standard values, no feasible linear programming solution existed, and the residuals of the non-negative least squares solution were high. As the uncertainties were made larger, the optimal value of total dense water formation grew toward $2 \mathrm{~Sv}$, not very different than the standard case. The extreme values widened quickly as the standard error values (multiplication factor 1) were approached, and continued to widen, though more slowly, as the error was increased beyond the standard values. At 5 times the standard values, zero dense water formation was an acceptable solution. By 10 times the standard values, the maximum consistent dense water formation had grown to $6 \mathrm{~Sv}$. This range was considerably wider than the standard, but still narrower than the $9 \mathrm{~Sv}$ range allowed when $\delta^{18} \mathrm{O}$ constraints were not used (Section 4.2.3).

Sensitivity to boundary forcing uncertainties As discussed in Section 3.5.1, uncertainties of the aggregate estimates may be too low. To determine the importance of this possibility, the solution was recalculated with the uncertainties of the aggregate estimates from Table 3.2 increased. Specifically, $\sigma_{Q}, \sigma_{T}, \sigma_{S}, \sigma_{O}$ (the 


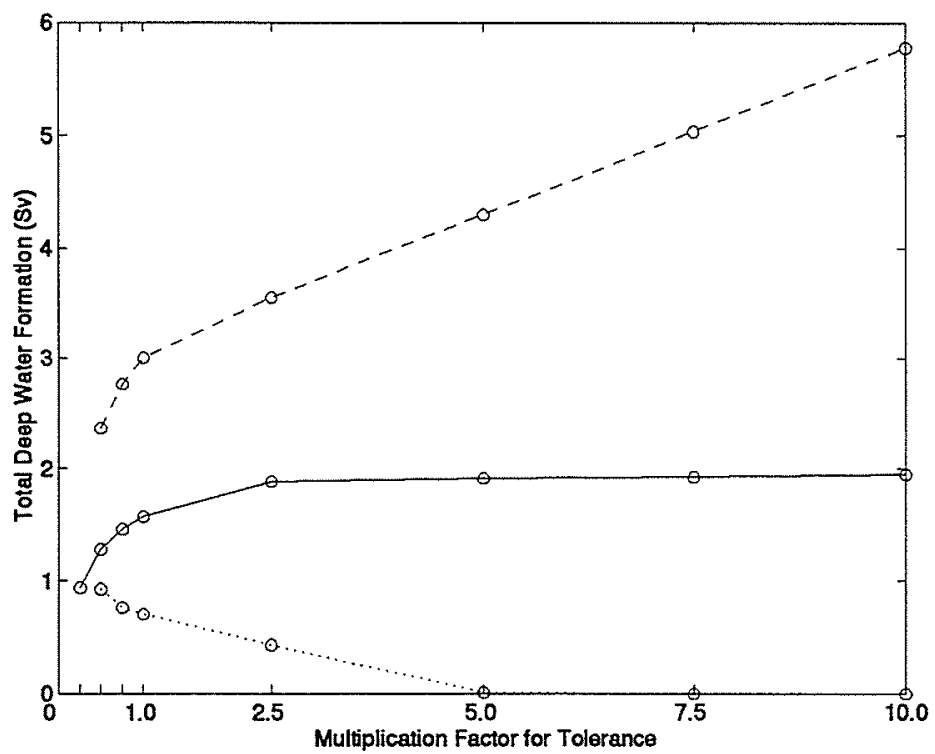

Figure 4.22: Minimum (dotted curve), maximum (dashed), and optimal (solid) dense water formation $v s$. the factor by which standard values for the prior error estimate of the conservation equations $\left(0.2 \mathrm{~Sv}\right.$ for volume, $0.3 \mathrm{~Sv}^{\circ} \mathrm{C}$ or $\mathrm{Sv} \cdot \mathrm{psu}$ for potential temperature and salt, $0.1 \mathrm{~Sv} \cdot \%$ for $\delta^{18} \mathrm{O}$ ) were multiplied prior to solution. No feasible linear programming solution existed for multiplication factors $\leq 0.25$.

standard error of the transport $\delta^{18} \mathrm{O}$ estimate), and $\sigma_{H}$ for the surface heat fluxes were all augmented by 50\%. These were used to calculate new mass-, heat-, and salt-conserving forcing with the method of Section 3.5.3, and that forcing was used to obtain a new solution.

The optimal solution with increased forcing uncertainties (not shown) was extremely similar to the solution with the regular uncertainties: only two of the internal fluxes differed in transport by more than $0.1 \mathrm{~Sv}$. Similarly, the Monte Carlo distributions of the optimal solution were very similar, regardless of the change in forcing uncertainty. The only significant effect of raising the forcing error was to reduce the minimum consistent volume of dense water formation (Table 4.5). 
Table 4.5: Minimum and maximum consistent amounts of dense water formation and surface mixed layer exchange in Sverdrups for the temperature-salinity- $\delta^{18} \mathrm{O}$ inversion, with normal and augmented forcing uncertainty.

\begin{tabular}{rcccc}
\hline & \multicolumn{2}{c}{ Dense Water } & \multicolumn{2}{c}{ Surface Mixed } \\
& \multicolumn{2}{c}{ Formation (Sv) } & \multicolumn{2}{c}{ Layer Exchange (Sv) } \\
& $\min$ & $\max$ & $\min$ & $\max$ \\
\hline regular uncertainty & 0.8 & 3.1 & 0.0 & 1.7 \\
augmented uncertainty & 0.2 & 3.6 & 0.0 & 2.1 \\
\hline
\end{tabular}

\subsection{Steady Solutions for Alternative Arctics}

I now turn to the question of whether the model would have steady solutions under conditions other than current Arctic conditions. If the amount of runoff or net ice export were to change, could dense water production on the shelves still maintain the current Arctic temperature and salinity profiles? If the Atlantic Layer were to warm, as may be happening (Carmack et al., 1995), would a steady solution at the warmer temperature still be possible?

The purpose of this exercise is to find the outer bounds of the set of steady solutions. When consistent, steady solutions exist, steady state is possible but not guaranteed: just because present profiles could be maintained under different conditions does not mean that they would be. Conversely, conditions for which no consistent, steady solution exists are guaranteed to bring about change in the Arctic. In any case, the steady model can not predict the end result of any change; it can only distinguish conditions in which change is possible from conditions where change is inevitable.

\subsubsection{Runoff volume}

The estimated runoff in the model was varied between 0 to $0.3 \mathrm{~Sv}$, bracketing the current estimate of $0.1 \mathrm{~Sv}$, with uncertainty held to a constant $0.03 \mathrm{~Sv}$. The 
amount of shelf-basin exchange in the steady solutions changed little over this range, except for the maximum consistent value of surface mixed layer exchange, which decreased from $2.0 \mathrm{~Sv}$ to $0.5 \mathrm{~Sv}$ as runoff increased. For runoff of $0.35 \mathrm{~Sv}$ and above, no steady solution was possible: the high runoff value made the net salt balance for the entire arctic system significantly negative. With the entire system freshening, it was impossible for every box to maintain its salinity.

One way to interpret this result is to note that the freshwater balance for the Arctic is so uncertain that the runoff can nearly quadruple before the net salt input is significantly less than zero. Looking at it another way, if the runoff were to quadruple and all other boundary fluxes were to keep their present values, the Arctic salinity profiles would have to evolve. This does not mean that the profiles might not evolve under a smaller change in runoff. A definite conclusion is that the amount of shelf-basin exchange required to maintain steady state is not highly sensitive to perturbations in the runoff.

\subsubsection{Net ice formation}

The net ice export estimate was varied from $0 \mathrm{~Sv}$ past the present estimate of $0.07 \mathrm{~Sv}$ to $0.15 \mathrm{~Sv}$, with the uncertainty maintained at $0.01 \mathrm{~Sv}$. From 0 to $0.09 \mathrm{~Sv}$ there was no significant change in the shelf-basin exchange. Between 0.09 and $0.15 \mathrm{~Sv}$, the minimum and maximum consistent values of dense water formation converged to 2.25 and $2.75 \mathrm{~Sv}$ respectively. When the net ice export was greater than $0.15 \mathrm{~Sv}$, no consistent solution was possible.

The competing needs of the shelf prevented a steady solution. As the ice formation increased, more and more salt was being left on the shelf, requiring more dense water flux to remove it. At the same time, more and more ${ }^{18} \mathrm{O}$ was being removed by fractionation upon freezing, making the remaining shelf water isotopically lighter. The dense water flux from the shelves only exacerbated the 
problem, by requiring a compensating mass flux from the isotopically light basin surface waters. Thus the model was caught: as ice formation increased, dense water formation had to increase to balance salt, but had to decrease to balance $\delta^{18} \mathrm{O}$. Once ice formation reached $0.15 \mathrm{~Sv}$, both demands were too great to be met simultaneously within the tolerance of the error bars. ${ }^{4}$

In short, the data cannot resolve any need for change in the property distribution of the Arctic water column should the net ice formation in the Arctic decrease. This is not to say there would be no change, simply that there wouldn't have to be. However, an increase of ice formation, in the absence of other forcing changes, would necessarily affect the temperature, salt, and/or $\delta^{18} \mathrm{O}$ distribution in the Arctic.

\subsubsection{Atlantic Layer temperature}

The temperatures of the WSC AW inflow, Atlantic layer boxes, and EGC AW outflow were increased together by increments ranging from 0 to $2^{\circ} \mathrm{C}$. To maintain the new profiles, the optimal value of surface mixed layer exchange dropped slightly as the temperature rose. At the same time, the amount of dense water formation increased slightly, in order to bring a greater volume of cold water to the Atlantic Layer. There were no significant changes in the extreme consistent values of shelfbasin exchange. In other words, the present data are not sufficient to determine the sensitivity of the system to changes in the Atlantic layer temperature.

\footnotetext{
${ }^{4}$ The model could meet both constraints by producing a smaller quantity of denser water, thus discharging more salt while drawing in less compensatory basin surface water. However, this would not supply adequate shelf water to the halocline.
} 


\subsection{Summary}

Given the current uncertainty in the boundary flux estimates, temperature and salinity data alone are not powerful enough to determine the shelf-basin exchange with any accuracy. In fact, they are not powerful enough to distinguish how much shelf-basin exchange occurs in the surface mixed layer, and how much via dense shelf overflows. This uncertainty is primarily due to lack of constraint of the surface circulation. Consideration of $\delta^{18} \mathrm{O}$ greatly ameliorates the problem, pinning the steady shelf-basin exchange down to a few tenths of a Sverdrup of off-shelf surface layer flow, from $0.1-0.7 \mathrm{~Sv}$ of dense water production in the Canadian Basin, and 0.7-2.4 Sv of dense water production in the Eurasian Basin.

Variations of the model assumptions have little effect on this main conclusion, but are individually revealing. Changes in the flow paths associated with the Arctic Archipelago outflow and the West Spitzbergen inflow influence the amount and density distribution of the dense shelf water required for steady state. However, changing the amount of runoff which crosses the basin without mixing has little effect on the amount of shelf-basin exchange required for steady state. Varying the amount of diffusion has little effect on the solution so long as the diffusion remains weak, but past a certain point, surface $\delta^{18} \mathrm{O}$ values cannot be maintained. Ice melt data are too uncertain to meaningfully constrain the model. The data are not sufficient to resolve the effects of small changes in the Arctic runoff or ice formation rates. However, should either greatly increase, no compensating effects exist which could maintain the existing distribution of temperature, salinity, and $\delta^{18} \mathrm{O}$. 


\section{Chapter 5}

\section{The Role of the Arctic Shelves: Revisiting Past Results}

\subsection{Introduction: the Distillery Shelf Model}

It is their many connections to the extra-Arctic which make Arctic shelves so interesting. Nonetheless, previous models of shelf-basin exchange (Killworth and Smith, 1984; Björk, 1989) disconnected the shelves from all sources and sinks except the Arctic basin, drastically simplifying their representation. ${ }^{1}$ The model shelves in these studies were simple circuits, existing only to accept water from the basin surface mixed layer, distill it by freezing out some fresh water, and return the resulting dense brine to the basin. Other shelf-basin exchanges, such as offshelf flow in the surface mixed layer, or shelfbreak upwelling, were omitted. Runoff and the Bering Strait were fed directly into the basin; the Barents Sea inflow was ignored. The Arctic Archipelago was ignored or merged into the East Greenland Current.

\footnotetext{
${ }^{1}$ The model of Rudels et al. (1994) and Jones et al. (1995) determines requisite shelf water properties without worrying about what sources feed the shelf or whether they are capable of producing the necessary shelf characteristics.
} 
These models' portrayal of the shelf as a simple distillery reveals an important preconception of the shelves' role as completely internal to the Arctic Ocean. That this conception is patently wrong does not automatically mean it is not useful: such simplifications are intrinsic to modeling, and when they render a problem more tractable without prejudicing the solution they are welcome. But this simplification completely changes the sources and sinks available to the shelves, and one might suspect it could influence the amount of shelf-basin exchange in steady solutions. As the following calculations will show, not only does adopting the distillery shelf model change the solution, it can in some cases preclude consistent solutions.

\subsection{Inversions with the Distillery Shelf}

The following sections describe inversions in which the box model of Chapter 2 was modified to include a distillery shelf. Specifically, surface mixed layer exchange was restricted to onshelf flow only. The Barents Sea inflow was ignored, as was the surface heat loss from the ice-free region of the Barents Sea. All other sources for the shelves were diverted to the basin, effectively parameterizing their passage across the shelves, as described below. No $\delta^{18} \mathrm{O}$ constraints were used in these calculations; this choice is discussed below.

Calculations were performed both with a one-basin Arctic (Section 5.2.1) and with the full two-basin representation (Section 5.2.2). The one-basin calculations allowed easy comparison to previous studies, which each used a single basin only. The two-basin version was then used to determine which of the one-basin results were due to the distillery shelf assumption, and which to the single-basin assumption. After these results are presented, they will be summarized (Section 5.2.3), and then discussed in terms of a simple toy model which explains the main features of the results (Section 5.3). As will be seen, the toy model results also shed some light on the full box model solutions of Chapter 4. 


\subsubsection{One basin}

The properties of the basin boxes were set by horizontally averaging the temperatures and salinities of Figure 2.2. A single shelf box was used, having the average properties of the two shelf boxes used earlier. ${ }^{2}$ Runoff ran directly to the surface mixed layer. The Bering Strait entered the upper halocline box, and the Arctic Archipelago and East Greenland Current Polar Water outflows were both drawn from the upper four boxes of the basin, i.e., from the surface mixed layer and the halocline. In addition to using the mass-, heat-, and salt-conserving boundary fluxes from Section 3.5.3 as usual, additional calculations were done using the simple aggregate estimates from Section 3.5.1. This made little difference to the one-basin results, but the simpler (i.e., unrotated) error structure of the aggregate estimates allowed clearer interpretation of solution residuals. ${ }^{3}$ For this reason, these additional calculations are the ones presented. The 9-box model yielded 39 constraints on 36 unknowns, with a rank of 34 . The nullspace was completely eliminated by the non-negative constraints, so all solution uncertainty was due to the uncertainty of the data.

As before, 1000 Monte Carlo realizations of the optimal solution were calculated. The modes and 95\% intervals for each flux are shown in Figure 5.1. The mode of the total dense water production was $1.7 \mathrm{~Sv}$, and the $95 \%$ interval was 0.7 $2.4 \mathrm{~Sv}$ (Figure 5.2), encompassing Björk's (1989) estimate of 1.0-1.5 Sv. Though the model allows dense water flux below the halocline, none appears in the solution.

Examination of the solution residuals (Figure 5.3) shows that this model neither clearly fits nor clearly contradicts the prior assumptions of steady state and of the

\footnotetext{
${ }^{2}$ In averaging, the approximation was made that each depth level of the Canadian Basin has the same volume as its Eurasian Basin counterpart, and that the two sets of shelves have the same volume as well. While the true volumes are not exactly equal (see Aagaard et al., 1985, Figure 2), they are close enough that errors introduced were small compared to the uncertainty of the solution.

${ }^{3} \mathrm{On}$ the rotation of the equations to ensure linear independence, and its implications for interpreting equation residuals, see footnote on page 92 .
} 


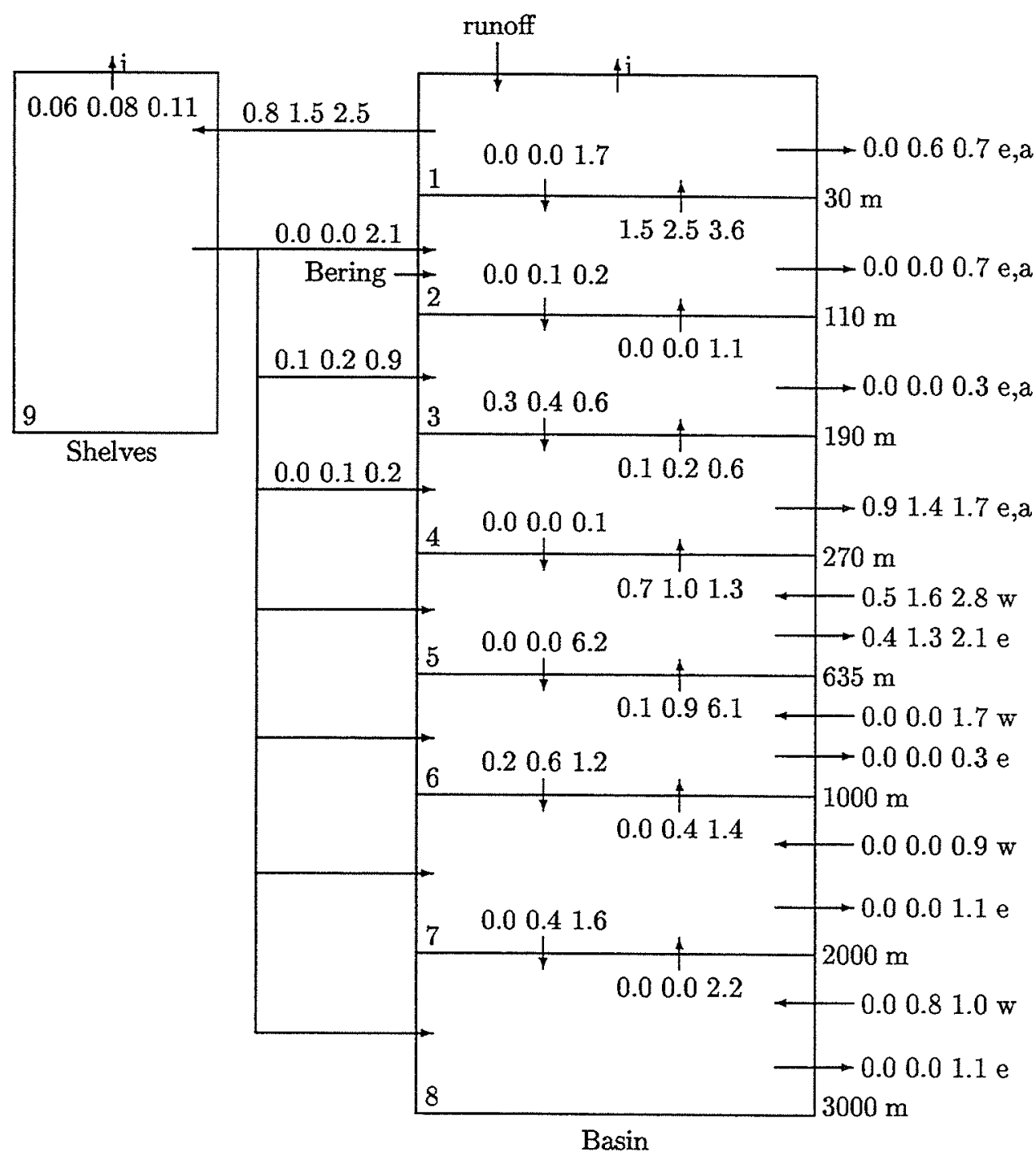

Figure 5.1: One-basin solution. Transports are written in Sverdrups as low mode high, where low and high are the lower and upper limits of the Monte Carlo $95 \%$ interval, and mode is the most likely value. Blank arrows indicate paths unused in the solution in at least $95 \%$ of the trials (i.e., 0.00 .00 .0 ). Water removed as ice is indicated by i, outflows through the Arctic Archipelago and East Greenland Current are marked a and e, and West Spitzbergen Current inflows are marked w. 


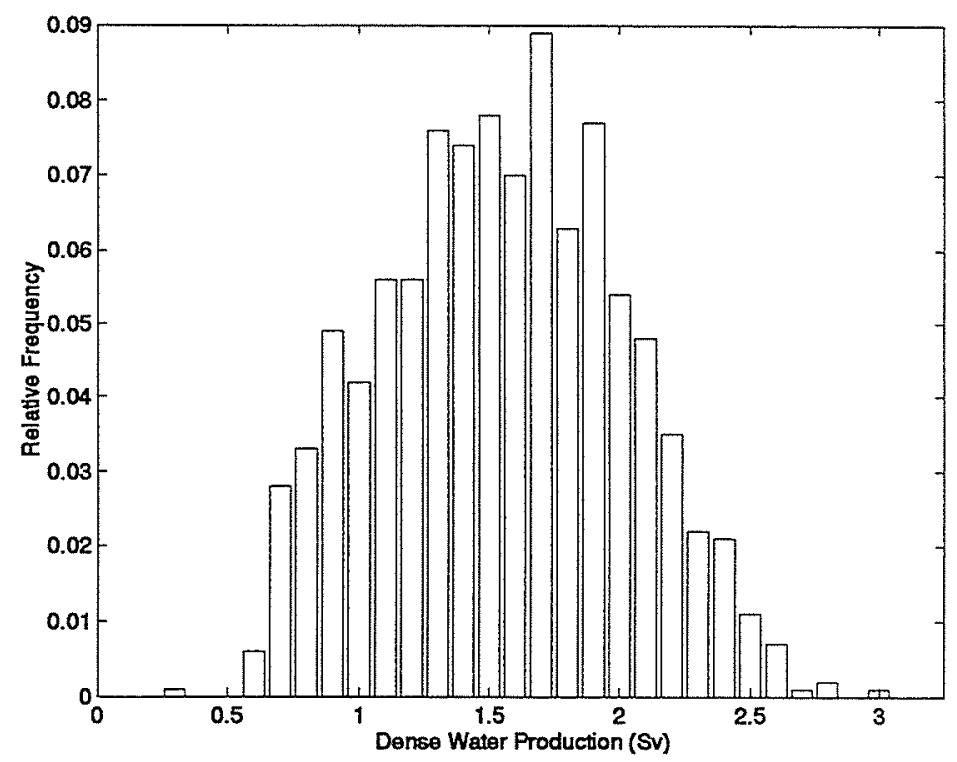

Figure 5.2: Histogram of 1000 Monte Carlo realizations of total dense water production in the one-basin model.

boundary flux estimates: only one of the most likely values for the residuals is beyond an "acceptable" 2 standard errors of zero, and with 39 equations, it is to be expected that one or two will be outside that range. In any case, the residuals are useful in pointing out the limitations of the one-basin distillery shelf model. The largest residual is in the equation specifying the outflow from the upper Arctic, equation index 29: the solution outflow (approximately $1.9 \pm 0.2 \mathrm{~Sv}$ ) falls short of the prior estimate $(2.6 \pm 0.3 \mathrm{~Sv})$ by between 1.5 and 4 standard errors. The variation is due primarily to the uncertainty in the prior estimate of the outflow: when the random Monte Carlo perturbation to the data sets the prior estimate higher, the shortfall is greater.

To investigate the model's inability to provide adequate outflow, the calculation was repeated with a strong weight (low uncertainty) ascribed to the upper Arctic outflow equation. In the resulting solution, the outflow constraint was satisfied, but the outgoing temperature flux was much too high. The upper Arctic outflow issued 


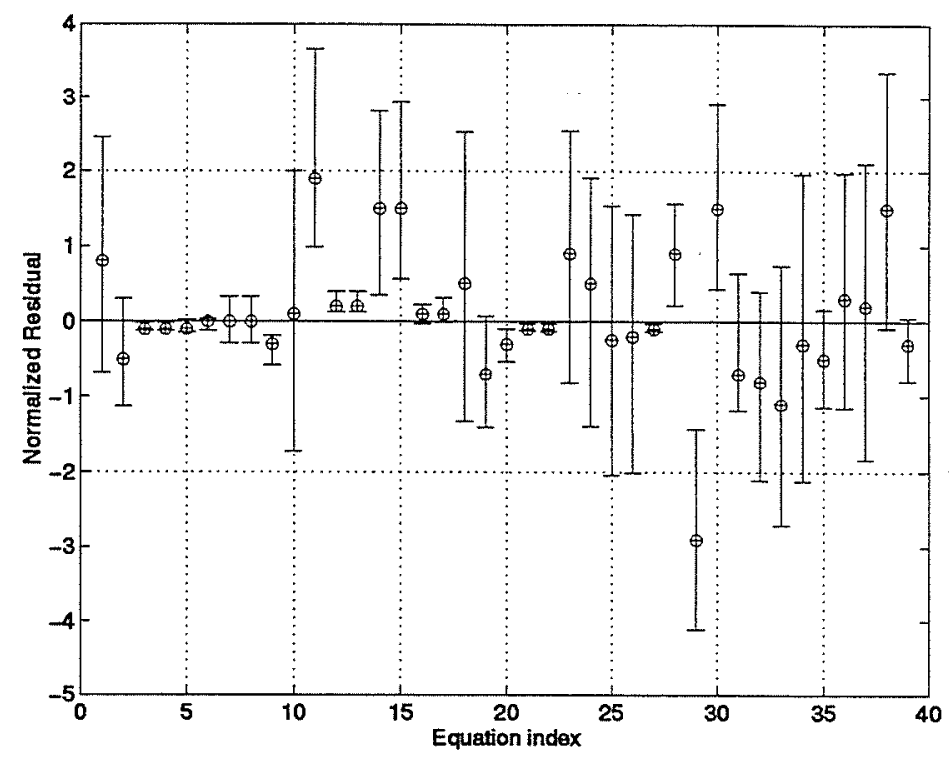

Figure 5.3: Monte Carlo modes (o) and 95\% intervals of the residuals for the scaled one-basin system. The residuals correspond to physical constraints as follows: 19 , volume conservation in boxes $1-9 ; 10-18$, potential temperature conservation; 19-27, salt conservation; 28 , total ice production; 29-31, total volume, temperature and salt outflow from boxes 1-4 (EGC PW and AA); 32-34, total volume, temperature and salt outflow from boxes 5 and 6 (EGC AW); 35-37, total volume, temperature and salt outflow from boxes 7 and 8 (EGC DW); 38, total inflow into boxes 5 and 6 (WSC AW); 39, total inflow into boxes 7 and 8 (WSC DW).

primarily from the lower, warmer halocline boxes, and not enough from the cold upper layers. The outgoing temperature flux equation was then also weighted. In the result, the two weighted equations were satisfied, but many of the other residuals became quite large. In short, the model was found to be able to satisfy the volume constraint, or the heat flux constraint, but not both.

Another constraint which the model found difficult to meet was heat conservation in box 2 (Figure 5.3, equation index 11). The solution does not send enough cold shelf water to box 2 to balance the warm Bering Strait input. 


\subsubsection{Two basins}

Next, the effect of resolving both basins (as in Figure 2.1) in the distillery-shelf solution was explored. For the two-basin solution, runoff flowed to the surface mixed layer boxes, the Bering Strait entered the upper Canadian Basin halocline box (box 2), and the Arctic Archipelago was drawn from the top four boxes of both the Canadian and Eurasian Basins, under a constraint that approximately three fourths flowed from the Canadian Basin as described in Section 2.6.2. The model was forced by the mass-, heat-, and salt-conserving boundary fluxes. In the calculations presented, no exchange was allowed between the two shelf boxes; when it was allowed, it made little difference.

\section{No interbasin exchange}

To start, no interbasin exchange was allowed, giving 70 equations in 68 unknowns. The rank of the system was 65 . In the solutions, all constraints were satisfied within two standard errors except for one: the model was again unable to supply enough EGC Polar Water outflow. The residual ranged from 2 to 5 standard errors too low with a mode of 3.5 .

\section{With interbasin exchange}

When interbasin exchange was allowed, the optimal solution (Figure 5.4) did not use it, except for surface mixed layer advection from the Canadian Basin to the Eurasian Basin of between 0 and $1 \mathrm{~Sv}$ (mode $0.3 \mathrm{~Sv}$ ). This is incidentally the same order of magnitude as the transpolar drift. The EGC PW transport residual subsided to between 1.0 and 3.7 standard errors (mode 2.0), comparable to the residuals in the one-basin case.

The $95 \%$ interval for total dense water formation was $0.5-2.8 \mathrm{~Sv}$ with a mode of $1.8 \mathrm{~Sv}$. As before, less dense water was formed in the Canadian Basin (mode 


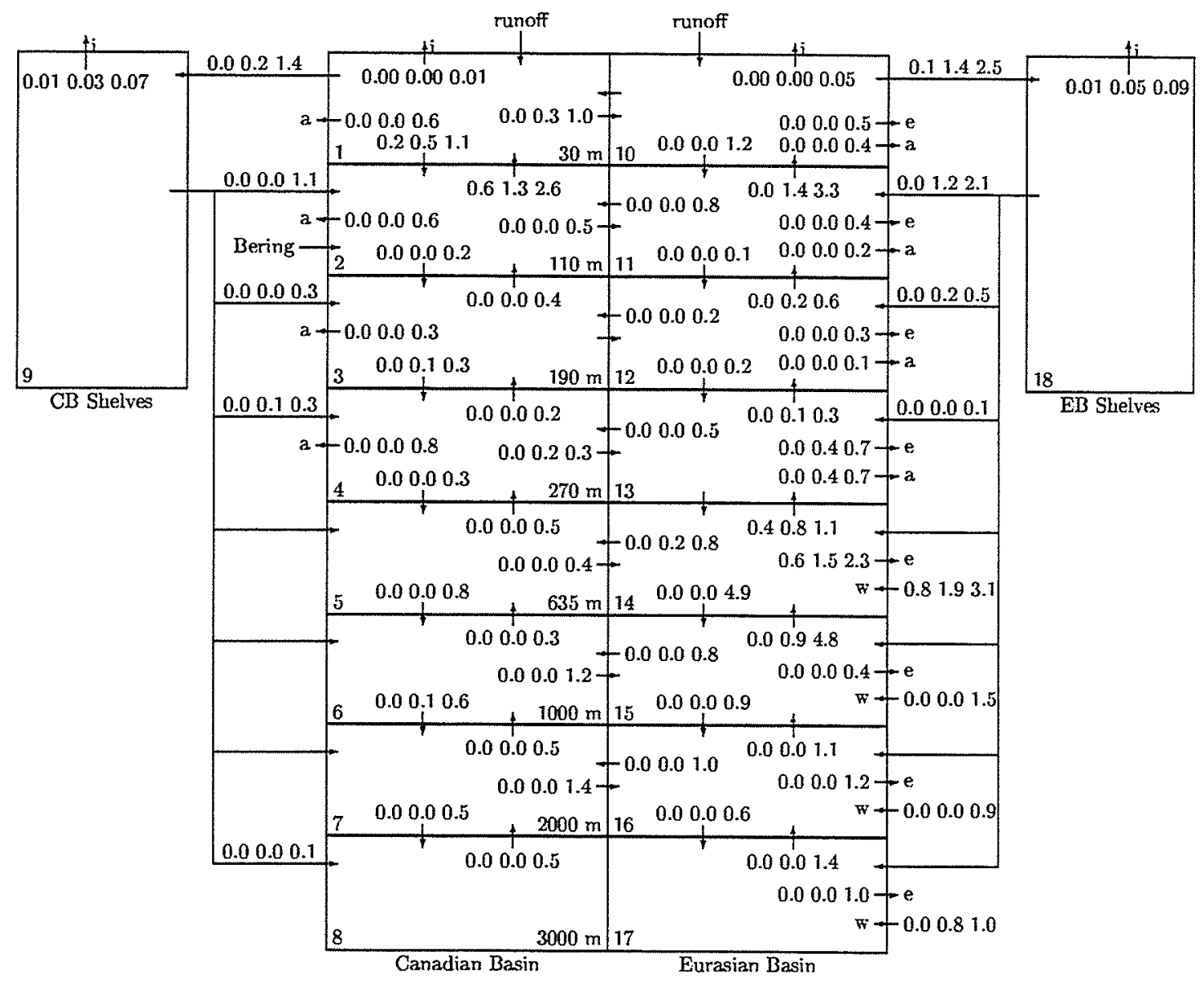

Figure 5.4: Two-basin solution for the distillery-shelf model, with inter-basin exchange allowed. Transports (in Sverdrups) are presented as in Figure 5.1. 


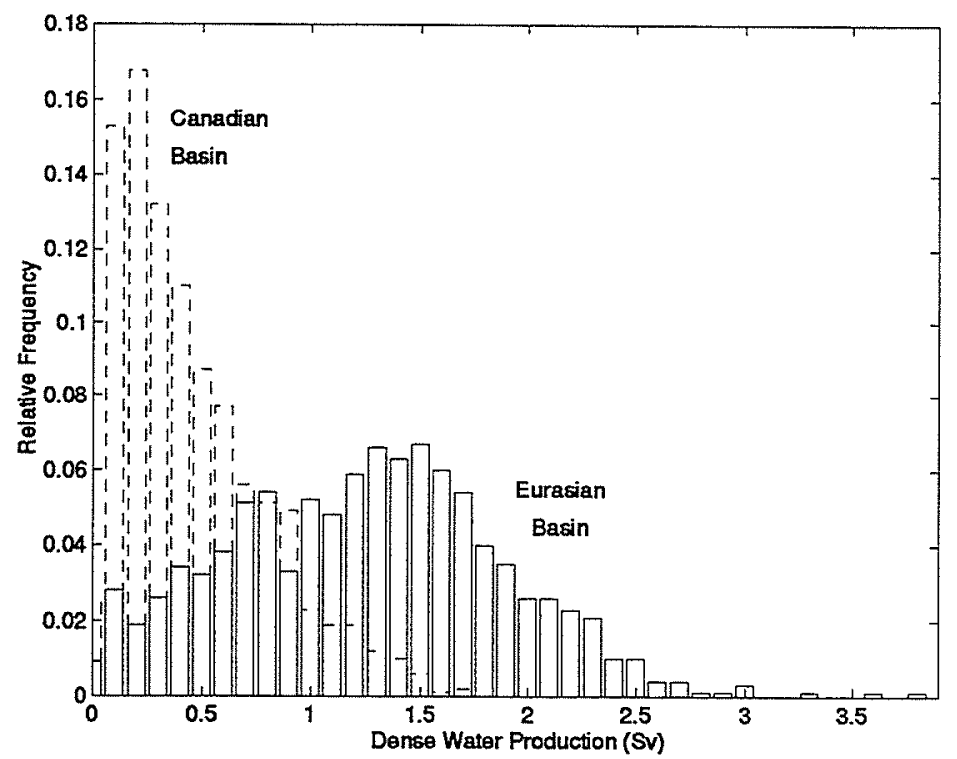

Figure 5.5: Histogram of 1000 Monte Carlo realizations of total dense water production in the two-basin distillery model, with interbasin exchange allowed.

$0.2 \mathrm{~Sv}, 95 \%$ interval 0.1-1.3 Sv) than in the Eurasian Basin (mode 1.5 Sv, 95\% interval $0.1-2.5 \mathrm{~Sv}$ ). The complete distribution of dense water formation in each basin is shown in Figure 5.5.

\subsubsection{Summary: distillery shelf inversions}

Adopting the distillery shelf model appeared to place a cap on the amount of cold outflow that the Arctic can produce in steady state. This cap was about halfway between the low and high likely values for the true output. The situation was roughly the same whether a one-basin or two-basin model was used, though the misfit became more acute when interbasin exchange was prohibited in the twobasin case. The one-basin model had some difficulty keeping the upper halocline sufficiently cool. In the two-basin model, the amount of dense water formation was greater on the Eurasian shelves; this was also the case with the standard shelf 


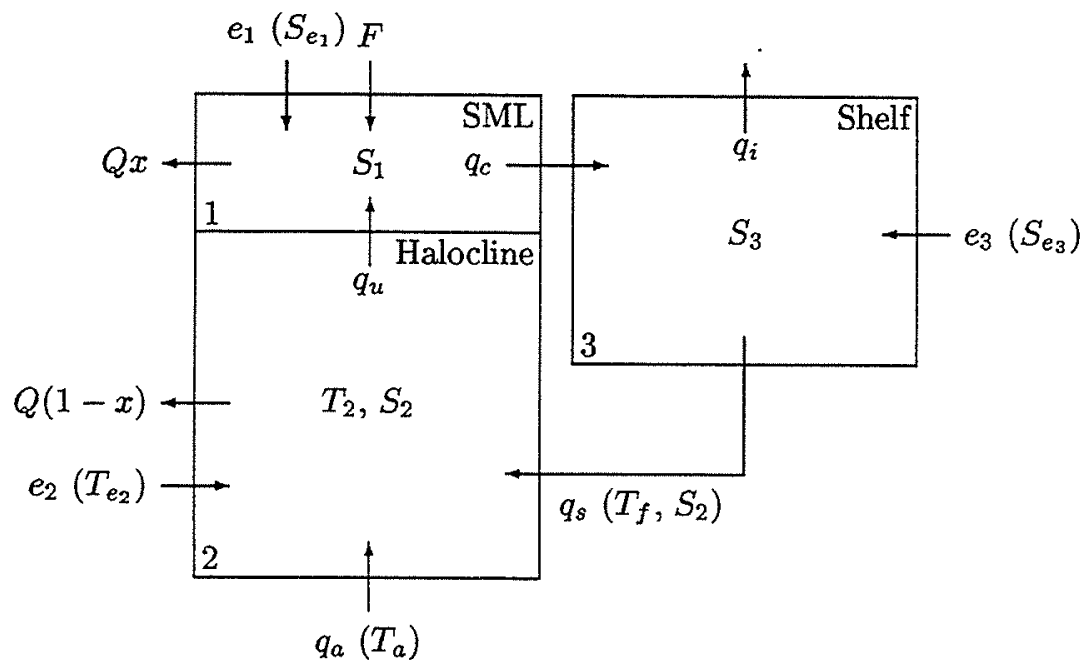

Figure 5.6: Toy model schematic.

model results of Chapter 4 . In the following two sections, an analytical toy model is first developed, and then used to interpret these results.

\subsection{A Toy Model}

To elucidate the constraints imposed by the distillery shelf, a toy box model has been constructed representing the interaction of the shelf, the halocline, and the upper Arctic outflow. The toy model consists of three well-mixed boxes (Figure 5.6): the surface mixed layer (box 1 , with salinity $S_{1}$ and freezing temperature $T_{f}$ ), the halocline (box 2 , with salinity $S_{2}$ and temperature $T_{2}$ ), and the shelf (box 3 , with salinity $S_{3}$ and freezing temperature). The system is fed from below by an infinite reservoir of Atlantic Layer water, with temperature $T_{a}$. It is forced by a flux $F$ of freshwater to the surface mixed layer, and also by a prescribed outflow $Q$ (representing the Arctic Archipelago and East Greenland Current outflows), of which a fraction $x$ comes from the surface mixed layer, and the rest from the halocline.

The model may further be forced by exterior sources $e_{1}$ to box 1 (salinity $S_{e_{1}}$ ), $e_{2}$ 
to box 2 (temperature $T_{e_{2}}$ ), and $e_{3}$ to box 3 (salinity $S_{e_{3}}$ ). By appropriate choice of the $e^{\prime} \mathrm{s}$, the shelf can be a simple distillery shelf $\left(e_{1}=e_{3}=0, e_{2}\right.$ represents the Bering Strait), or a receiver of external sources $\left(F=e_{2}=0, e_{3}\right.$ represents runoff plus the Bering Strait and/or the Barents Sea inflow). If the model is used to represent the Eurasian Basin, then $e_{1}$ represents the transpolar drift from the Canadian Basin, and $e_{3}$ the Barents Sea inflow. By setting all three $e^{\prime} s$ to zero, hypothetical solutions for the distillery shelf without the Bering Strait can be explored. Several such combinations are used and detailed below.

Model unknowns include the vertical distribution of the outflow $x$, and the internal fluxes: $q_{a}$ from the Atlantic Layer to box $2, q_{u}$, the upwelling from box 2 to box $1, q_{i}$, the freshwater removed as ice from box $3, q_{s}$, the dense shelf water which sinks to box 2 , and $q_{c}$, the compensating inflow to the shelf from box 1 . All internal and outgoing fluxes carry the temperature and salinity of their box of origin, except for $q_{i}$ and $q_{s}$. The latter is formed by brine rejection at the freezing point $T_{f}$. It sinks to its density level, or, nearly equivalently at low temperatures, its salinity level. Since by definition it is the water which sinks to box 2 , its salinity is approximately $S_{2}$.

At this point the toy model contains six unknowns. If a straightforward analytic solution is to be found, six constraints will have to be applied. But there are many more than six relevant constraints to choose from-mass, heat, and salt conservation in each box alone give nine. Instead of choosing, I could add more unknowns, such as downwelling from the mixed layer, until the degrees of freedom caught up to the constraints. Both these paths, followed to their ends, lead straight back to the full box model. Rather than going down that road (which, after all, I have just spent two chapters traveling), I choose to halt here at six unknowns.

How, then, to choose which six constraints? I choose the six constraints which represent, as simply as possible, the demands of brine production while maintaining a fresh surface layer and a cold halocline. They are: mass conservation in each 
box,

$$
\begin{aligned}
q_{u}+F+e_{1} & =Q x+q_{c} \\
q_{a}+q_{s}+e_{2} & =Q(1-x)+q_{u} \\
q_{c}+e_{3} & =q_{i}+q_{s},
\end{aligned}
$$

salt conservation in the surface boxes,

$$
\begin{array}{r}
q_{u} S_{2}+e_{1} S_{e_{1}}=\left(Q x+q_{c}\right) S_{1} \\
q_{c} S_{1}+e_{3} S_{e_{3}}=q_{s} S_{2}
\end{array}
$$

and temperature conservation in the halocline,

$$
q_{a} T_{a}+q_{s} T_{f}+e_{2} T_{e_{2}}=\left[Q(1-x)+q_{u}\right] T_{2}
$$

Whether this is a useful choice of constraints remains to be seen: it will have been useful if the resulting toy model solution is able to illuminate the results of the full least-squares box model.

The solution is

$$
\begin{aligned}
q_{s} & =\frac{Q-\left(F+e_{1}+e_{2} \Delta_{2}+e_{3} \Delta_{3}\right)}{\Delta_{T}-\Delta_{S}} \\
q_{c} & =q_{s} \Delta_{S}-e_{3} \Delta_{3} \\
q_{i} & =q_{s}\left(\Delta_{S}-1\right)-e_{3}\left(\Delta_{3}-1\right) \\
q_{a} & =q_{s}\left(\Delta_{T}-1\right)+e_{2}\left(\Delta_{2}-1\right) \\
q_{u} & =\frac{\left(Q-e_{2} \Delta_{2}\right) \Delta_{S}-\left(F+e_{1}+e_{3} \Delta_{3}\right) \Delta_{T}}{\Delta_{T}-\Delta_{S}}+Q x \\
x & =\frac{\Delta_{S}}{\Delta_{T}-\Delta_{S}}\left[\frac{F\left(\Delta_{T}-1\right)+E}{Q\left(\Delta_{S}-1\right)}-1\right]
\end{aligned}
$$

where $\Delta_{T}=\left(T_{a}-T_{f}\right) /\left(T_{a}-T_{2}\right), \Delta_{S}=S_{2} / S_{1}, \Delta_{2}=\left(T_{a}-T_{e_{2}}\right) /\left(T_{a}-T_{2}\right), \Delta_{3}=$ $S_{e_{3}} / S_{1}$, and, with $\Delta_{1}=S_{e_{1}} / S_{1}, E$ is the linear combination of external sources

$$
E=e_{1}\left[\Delta_{1}-1+\Delta_{T}\left(1-\Delta_{1} / \Delta_{S}\right)\right]+e_{2} \Delta_{2}\left(\Delta_{S}-1\right)+e_{3} \Delta_{3} \Delta_{T}\left(1-1 / \Delta_{S}\right) .
$$


Table 5.1: Toy model parameters. Flux values are based on Table 3.4

\begin{tabular}{crrcrr}
\hline parameter & \multicolumn{2}{c}{ value } & parameter & \multicolumn{2}{c}{ value } \\
\hline$Q$ & 2.8 & $\mathrm{~Sv}$ & $Q_{\text {Bering }}$ & 0.8 & $\mathrm{~Sv}$ \\
$F$ & 0.1 & $\mathrm{~Sv}$ & $T_{\text {Bering }}$ & -0.5 & ${ }^{\circ} \mathrm{C}$ \\
$S_{1}$ & 31.6 & & $S_{\text {Bering }}$ & 32.5 & \\
$S_{2}$ & 33.7 & & & & \\
$T_{2}$ & -1.0 & ${ }^{\circ} \mathrm{C}$ & $Q_{\text {Barents }}$ & 1.5 & $\mathrm{~Sv}$ \\
$T_{f}$ & -1.8 & ${ }^{\circ} \mathrm{C}$ & $S_{\text {Barents }}$ & 35.0 & \\
$T_{a}$ & 0.5 & ${ }^{\circ} \mathrm{C}$ & & & \\
\hline
\end{tabular}

An important feature of the solution is the strength of the upwelling $q_{u}$. Consider the case where there are no external oceanic sources; i.e., an Arctic with a distillery shelf and no Bering Strait, as was considered by Killworth and Smith (1984). In that case, $e_{1}=e_{2}=e_{3}=0$, and if $F \Delta_{T}$ is small compared to $Q \Delta_{S}$,

$$
q_{u} \approx Q\left(\frac{\Delta_{S}}{\Delta_{T}-\Delta_{S}}+x\right) .
$$

For typical Arctic values (Table 5.1), the upwelling is 2.75-3.75 times the outflow, depending on $x$. The strong upwelling can be explained in terms of a "circuit of need:" As halocline water outflows, it must be replaced. This requires dense water, which drains the shelf. The shelf is replenished by taking water from the surface mixed layer; the surface mixed layer, in turn, draws water up from the halocline. Now, more halocline water must be replaced, which starts the process again. This very strong upwelling at the base of the surface mixed layer appeared in the numerical model results of Killworth and Smith (1984, Figure 7), though it was not explicitly discussed.

Strong upwelling tends to increase the salinity of the surface mixed layer, and is therefore limited by the strength of the freshwater input $F$. However, (5.11) shows that the amount of upwelling in the steady solution is reduced by the presence of any external source, i.e., any non-zero e. External sources of mass help to assuage the demand circuit established by the export of halocline water. Steady solutions 
are therefore possible only if external sources reduce the upwelling to a point where the surface salinity balance can be maintained by runoff. This is expressed in the toy model by requiring that $0 \leq x \leq 1$, yielding a solvability condition on $Q, F$, and $E$ :

$$
1 \leq \frac{F\left(\Delta_{T}-1\right)+E}{Q\left(\Delta_{S}-1\right)} \leq \frac{\Delta_{T}}{\Delta_{S}}
$$

(assuming $\Delta_{T}>\Delta_{S}>1$, as is the case for the Arctic). Using the parameters from Table 5.1 gives approximately $0.20 \leq(F+2 E) / Q \leq 0.28$. In the Arctic, $F / Q$ alone is approximately 0.04. A further source of mass, i.e., non-zero $E$, is clearly necessary for steady solutions to exist.

This same conclusion was drawn by Killworth and Smith (1984) from their numerical model. They found steady solutions with approximately accurate temperature and salinity structure only after adding the Bering Strait inflow, parameterizing its passage across the Chukchi shelf by directly inserting it in the halocline. In the toy model, this is accomplished by setting $e_{1}=e_{3}=0$ and setting $e_{2}, T_{e_{2}}$ and $S_{e_{2}}$ to values appropriate for the Bering Strait inflow (Table 5.1). This raises $(F+2 E) / Q$ to about 0.07 , still too low for a feasible solution. (Killworth and Smith's solution included a larger Bering Strait volume, a much greater temperature contrast between the Bering Strait water and the upwelling Atlantic Layer Water, and a slightly smaller output volume.) If instead, it is acknowledged that the Bering Strait first enters the Arctic via the Chukchi shelf, ascribing the Bering inflow to the shelf input $e_{3}$ gives $(F+2 E) / Q=0.11$ : still too low, but closer to feasibility. Combining the Bering and Barents fluxes to the shelves as $e_{3}$ gives $e_{3}=2.3 \mathrm{~Sv}, S_{e_{3}}=34.1$, yielding $(F+2 E) / Q=0.28$, just within the feasible range. If $Q$ were any larger, consistent solutions would not be possible; as it is, only by properly representing external sources as flowing to the shelves can steady solutions be found.

Many important degrees of freedom have been omitted from the toy model. The 
toy model does not resolve the distinctive vertical structure of the upper Arctic, nor the gradients between the Canadian and Eurasian basins. Many exchanges, notably diffusion, are not parameterized in the toy model, and other exchanges are oversimplified: the Arctic Archipelago outflow, for example, is in fact drawn in part from the Canadian Basin shelves, affecting the values of $Q$ and $e_{3}$. Most importantly, uncertainties in the flux estimates make the feasible range (5.14) somewhat elastic, and they determine which constraints have priority in the event of contradictions. Several key constraints have also been ignored. Can heat be conserved in the surface boxes? Can the Atlantic Layer supply $q_{a}$ while still conserving mass, heat, and salt? Another important constraint is provided by an estimate of the outflow transport temperature $T$, which translates into an additional constraint on $x:$

$$
T_{1} x+T_{2}(1-x)=T
$$

This may contradict the value of $x$ given in (5.12); in fact, this contradiction is very important in explaining the results of the distillery model least-squares inversions (see below). All of these deficiencies amply illustrate the need for the full leastsquares treatment used in previous sections.

There are two conclusions from the toy model. First, external sources are necessary to steady solutions, in order to reduce the amount of salt upwelled to the surface mixed layer. Second, inputs to the shelves are more efficient than inputs to the basin at reducing the upwelling required for steady state. These conclusions have some value for interpreting the distillery model inversions of the previous section. This is taken up in the following discussion.

\subsection{Discussion}

The largest residuals of the one-basin solution of Section 5.2.1 were due to the model's inability to provide enough outflow, or to adequately cool the upper halo- 
cline. When the equation weights were changed to insist on a high outflow, the outflow became too warm. These results are easily explained in terms of the toy model.

Several constraints were at odds: on the one hand, salt conservation in the surface mixed layer permitted only as much upwelling as can be balanced by the freshwater input. On the other hand, the high prior estimate of outflow (high $Q$ ) and the low prior estimate of outgoing heat flux [high $x$, through (5.15)] together required a large amount of upwelling (5.11). Of these constraints, the prior estimate of outflow $Q$ had the largest uncertainty, and therefore received the lowest priority in the least-squares calculation. The total outflow was thus restricted, in order to keep the upwelling manageable.

With $Q$ thus limited, dense water production was also limited by (5.7), and the upper halocline warmed under the unchecked influence of the Bering Strait. (The solution prioritized salt conservation in the surface mixed layer over heat conservation in the upper halocline because the latter's uncertainty included the large errors in the Bering Strait heat flux estimate, while the former's uncertainty contained only the relatively small error of the freshwater flux.) When the weight of the transport estimate was increased, forcing the outflow toward the high prior estimate, the accompanying increase in upwelling was repressed by lowering the fraction of outflow from the cold upper boxes, i.e., reducing $x$. The price was that the average temperature of the outflow was higher than the prior estimate.

The findings from the toy model also help explain the two-basin distillery shelf inversions (Section 5.2.2). When no interbasin exchange was allowed, all constraints concerning the Canadian Basin were met without trouble, but the Eurasian Basin outflow was 2-5 standard errors too low. The reason for this problem is now clear: isolated from the Canadian Basin and the Bering Strait, the Eurasian Basin had no external source to reduce the need for upwelling. The outflow demands placed on the Eurasian Basin by the East Greenland Current are much harder to 
satisfy than those placed on the Canadian basin by the Arctic Archipelago, because the East Greenland Current is both larger and colder than the Archipelago outflow. This is why the large residual appeared in the Eurasian Basin and not in the Canadian.

When interbasin exchange was permitted, the surface mixed layer "transpolar drift" to the Eurasian Basin provided the missing external source, taking the role of $e_{1}$ in the toy model, and reduced the outflow residual. From the point of view of the Canadian Basin, the surface drift added to the outflow, making a steady solution more difficult. However, since the demands of the Archipelago outflow on the Canadian Basin were not very onerous, the Canadian Basin was able to afford the loss.

While it would be perfectly feasible to add $\delta^{18} \mathrm{O}$ or other constraints to the distillery model, as was done for the full model in Chapter 4, there is no real reason to do so. The distillery model had trouble finding solutions consistent with the constraints already upon it; adding more could only increase the misfit.

The toy model illustrates that Polar Water output is limited no matter what the shelf model; the limits are just stricter with the distillery shelf model than with shelves open to external sources. In Chapter 4, where the full, open shelf model was used, the limits were approached in two cases. In Section 4.2.3, no shelf-basin exchange was allowed, and no consistent solution was found. Without the ability to replace outflowing upper waters, one of the constraints the model could not meet was the requirement for cold outflow. Section 4.2.4 explored the sensitivity to the prescribed temperatures and salinities in the model. Of all the $\mathrm{T} / \mathrm{S}$ combinations tried, the only ones for which there was no consistent solution were those with high basin temperatures and low surface salinity. The toy model explains the difficulty of this combination. The low surface salinity meant that upwelling was even less tolerable. At the same time, the higher basin temperatures meant that providing cold average output required more discharge from the relatively cold surface layer, 
which increased the need for upwelling-an unresolvable conflict.

The toy model provides one further insight, perhaps already apparent, about both the full model inversions of Chapter 4 and the two-basin distillery shelf inversions of this chapter. In both cases, more dense shelf water was required in the Eurasian Basin than in the Canadian. Apparently, this is simply because more Polar Water is drawn from the Eurasian Basin, so more shelf water is needed for its replacement. This is loosely quantified by (5.7) of the toy model, which shows that the dense shelf water $q_{s}$ is proportional to the Polar Water outflow $Q$.

\subsection{Summary}

The distillery shelf model places a limit on the amount of Polar Water outflow which can be consistently produced in steady state. This limit is in about the middle of the range of likely values for the true output. If one were to ask if the distillery shelf model fit the data, the answer would have to be that it might, depending on whether the true Polar Water output is above or below the present estimate. But this question is really beside the point: even if the distillery model fits the data, it is still wrong, since external sources are known to feed the shelves.

Perhaps a more important question is whether adopting the distillery model as a simplification significantly affects the solution. The answer to that question is clearly, "yes." By restricting the sources of water for the shelves, the distillery model greatly magnifies the upwelling which accompanies the replacement of outflowing Polar Water. This excessive upwelling, which greatly limits the range of consistent solutions, is no more than an artifact of an unnatural simplification. This is reason enough to recommend that future models not rely on the distillery shelf assumption.

There is a second, less rigorous reason for avoiding the distillery shelf, which is nonetheless as important. Simple models are seductive. Compact and accessible, 
they easily become the central image brought to mind when the systems they represent are considered. As a result, aspects of the system not present in the model are more likely to be set aside or forgotten in subsequent studies. For the sake, then, of subsequent research, even simple representations should strive to include the most conceptually important aspects of the system. The Arctic shelves actively mediate between the Arctic and the extra-Arctic. When one thinks of the Arctic shelves, the first and foremost impression should not be of an internal, closed-loop distillery. It should be of heavy traffic: two-way exchanges between the shelves and the basins, two-way exchanges between the shelves and the world ocean. 


\section{Chapter 6}

\section{Summary and Discussion}

\subsection{Summary of Results}

A current question in Arctic modeling is the relevance of assuming a steady state. While the assumption may always be justifiable as a theoretical simplification and a baseline for further studies, it will be quantitatively viable only so long as it does not lead to contradictions. The compilation of boundary flux estimates and the calculation of consistent, steady circulation schemes demonstrate that at present, the available data are insufficient to reject the steady state hypothesis.

Assuming a steady state, this work concurs with the main conclusion of Björk (1989), viz., the observed Arctic T/S profiles can be maintained in steady state by a flux of 1-2 Sv of dense shelf water to the basins. However, the present work departs from previous estimates in three major ways. First, the two-basin structure of the Arctic is resolved. Second, the solutions are constrained by independent estimates not only of the inflows, but the outflows as well, and the uncertainties of these constraints are accounted for when estimating the solution. Third, the two-way exchanges between the shelves and the basins, and between the shelves and the extra-Arctic, are represented, allowing the full range of steady solutions consistent 
with the data to be examined. Due directly to these differences, several important conclusions arise:

1. In steady state, there is a net flux from the shelves to the basins. Budgets of the boundary flux alone (Section 3.6) show there must be a net flux from the shelves to the basins of $1.2 \pm 0.4 \mathrm{~Sv}$.

2. In steady state, water flowing from the shelf is replaced not from the basin, but from external sources. The optimal model solution, i.e., the circulation which most closely satisfies the full suite of box model constraints (Section 4.3.1) includes zero flux from the basin to the shelves, and $1.8 \mathrm{~Sv}$ from shelf to basin (including both dense overflows and surface drift).

3. In steady state, most shelf-basin exchange is in the form of dense shelf water. Of the $1.8 \mathrm{~Sv}$ flowing to the basin in the optimal solution, $1.4 \mathrm{~Sv}$ is dense shelf water, and only $0.4 \mathrm{~Sv}$ flows in the surface mixed layer. This result is wellconstrained: the $\delta^{18} \mathrm{O}$ data establish that the surface mixed layer advection runs principally from shelf to basin, in mere tenths of Sverdrups, in all the solutions permitted within the flux uncertainties (Figure 4.12).

4. In steady state, dense water is formed principally on the Eurasian Basin shelves. Of the 1.4 Sv of dense water formed in the optimal solution, 1.3 Sv is in Eurasian Basin. All calculations in which the two Arctic basins are separately resolved exhibit the same tendency.

5. Estimates of shelf-basin exchange based only on mass, heat, and salt conservation are not well constrained by the available data. Without other constraints such as $\delta^{18} \mathrm{O}$ conservation, the observed temperature and salinity profiles can be maintained in steady state within error bars either by dense shelf-basin flux alone, or by surface mixed layer shelf-basin flux alone. The solution of 
minimum residual includes a dense water flux of $1.9 \mathrm{~Sv}$, close to previous estimates, but with higher uncertainty: the $95 \%$ confidence interval for optimal total dense water formation is 1.2 to $4.6 \mathrm{~Sv}$. Moreover, solutions which are steady within error bars can consistently have as much as $9 \mathrm{~Sv}$ of dense water production. The surface mixed layer exchange is even more uncertain: solutions steady within error bars are possible even with an unlikely $70 \mathrm{~Sv}$ of surface mixed layer exchange. This high uncertainty is due primarily to uncertainty in the surface fluxes and circulation.

6. Inclusion of $\delta^{18} O$ conservation requirements constrains the estimates of steady shelf-basin exchange. The required constraints on the surface circulation are provided by conservation statements for $\delta^{18} \mathrm{O}$. Their inclusion restricts the surface mixed layer transport to a small shelf-to-basin drift, with a maximum of $0.7 \mathrm{~Sv}$. The optimal dense water formation is reduced to $1.6 \mathrm{~Sv}$, with a confidence interval of 1.1-2.2 Sv. The maximum consistent value is reduced to $3.1 \mathrm{~Sv}$. Most importantly, the $\delta^{18} \mathrm{O}$ constraints show that the dense water flux is necessary, with a minimum consistent value of $0.8 \mathrm{~Sv}$. These totals are not terribly sensitive to the exact choice of $\delta^{18} \mathrm{O}$ profiles. Estimates of shelfbreak upwelling are also well-constrained, though less so: upwelling to the shelf from the lower halocline is not required for steady state, and cannot consistently be greater than $3.8 \mathrm{~Sv}$. Further, its inclusion in the model did not drastically affect the range of dense water formation or surface mixed layer exchange consistent with steady state.

7. Several factors affecting steady shelf-basin exchange cannot be determined with this model and the available data. The data-model combination was not able to distinguish whether the Canadian Basin or Canadian Shelves were the source of the Arctic Archipelago outflow, nor could it determine the vertical distribution of the entering West Spitzbergen Current Atlantic Water. (The 
choices made regarding the representation of these flow paths did, however, slightly influence the amount and/or distribution of dense shelf water required for steady state.) Solutions with runoff routed to the shelf, to the basin, or some of each were all equally consistent. The spatial distribution of ice formation and melt, as well as advection patterns, were also indeterminate, due to the large error bars associated with the ice data on these scales. Because of their large uncertainty, the ice information was downweighted in the model calculations and did not greatly affect the amount or nature of shelf-basin exchange.

8. The distillery shelf assumption unnaturally limits the range of shelf-basin exchange consistent with steady state. In particular, if the outflow from the upper Arctic is any greater than a threshold value (which is close to the present estimate), no distribution of dense shelf water can meet the steady state demand. Only by recognizing the connections between the shelves and external sources can consistent solutions be found over the whole range of plausible transports across the Arctic boundary.

These results are next discussed in light of previous studies.

\subsection{The Steady Arctic}

The full model with $\delta^{18} \mathrm{O}$ constraints estimates the total Arctic dense water production to be 1.1-2.2 Sv. That this result is so similar to the range estimated by Björk (1989) is remarkable, considering the large number of differences between his premises and those adopted here. Essentially, his model and the current one face the same problem: the indeterminacy of the shelf-basin exchange from mass, heat, and salt conservation alone. Björk reduces the uncertainty by reducing the degrees of freedom, such as two-way surface mixed layer flow, and the number of 
mass sources for the shelves. The present study reduces uncertainty by adding independent constraints, viz., $\delta^{18} \mathrm{O}$ conservation and outflow values. Though both techniques produce the same overall transport value, their respective solutions do differ in important ways. In particular, the present model is robust to the potentially high values of Arctic outflow. Conversely, in Björk's model, no consistent solution can produce outflow rates as high as those observed. This belies Björk's claims that the distribution of dense water salinities in his model can be tuned exactly to fit the observed temperature and salinity profiles. ${ }^{1}$

To what extent can one expect tracer distributions to constrain steady circulations? LeGrand and Wunsch (1995) have demonstrated that a "standing crop" of passive tracers alone cannot constrain circulation without some sort of ratesetting rule, or "clock." In their North Atlantic model, circulation rates are set by geostrophy, but the present estimates focus on cross-shelfbreak flows, where the geostrophic approximation fails and no simple physical rate-setting rule exists. Instead, the "clock" is provided by the prior estimates of transport rates across the system boundaries. Yet this rate information, along with temperature and salinity distributions, is still inadequate to constrain the shelf-basin exchange. Why?

The large uncertainty that appears when the surface circulation is not well constrained (Section 4.2.2) suggests the answer. In the surface layer, temperature gradients are very weak, so heat conservation constraints do little to limit the range of consistent circulation. Salinity gradients are somewhat stronger, but their constraining effect is weakened by the large uncertainty in the prescribed salt fluxes, and the multiple degrees of freedom affecting the salt budgets. (The consideration of ice melt and advection in Section 4.4 .6 compound this problem by adding more degrees of freedom than constraints.) So the tracer information in the surface layers

\footnotetext{
${ }^{1}$ Björk (1990) adds $\delta^{18} \mathrm{O}$ to his model to verify the accuracy of his earlier (1989) solution. He finds that his model is capable of recapturing the observed profiles, except for a persistent underestimate of the $\delta^{18} \mathrm{O}$ in the mid-halocline.
} 
was not capable of constraining the circulation. Conservation of $\delta^{18} \mathrm{O}$, which varies strongly between shelves and basin, provided the needed "orthogonal information" (Bauch, 1995) to reduce the solution uncertainty.

Resolving the two-basin structure of the Arctic reveals a strong asymmetry in total dense water production. There are two ways of interpreting this result: a "push" view, in which overflows alleviate mass forcing to the shelf, and a "pull" view, in which dense water is formed to meet the steady-state needs of the basin. From the first point of view, consider that the Eurasian Basin shelves are forced by $1.5 \mathrm{~Sv}$ of inflow from the Norwegian Sea. That input has to go somewhere. The Canadian Basin shelves are forced by only $0.8 \mathrm{~Sv}$ of Bering Strait inflow, much of which is balanced by outflow through the Arctic Archipelago. From mass balance alone, then, the Eurasian Basin shelves ought to export more water to the basins.

From the second viewpoint, the asymmetry between basins of the amount of dense water formation can be considered in terms of flushing times in the basins. In steady state, Eurasian Basin halocline water is drained by the East Greenland Current. It is also depleted by upwelling to the surface mixed layer, as part of a complicated balance with the surface circulation and with the uncertain and potentially large surface fluxes. Replacing this lost halocline water requires a large input from the Eurasian Basin shelves. Additionally, the deep Eurasian Basin appears to be flushed by the deep West Spitzbergen Current inflow and East Greenland Current outflow. Maintenance of the deep $\delta^{18} \mathrm{O}$ deficit noted by Bauch et al. (1995), therefore, requires a means to transport river runoff to depth, viz., shelf production of very dense water. In the Canadian Basin, on the other hand, little upwelling from the halocline is called for. Canadian Basin halocline waters are undisturbed by boundary forcing, because the primary sources and sinks-runoff, the Bering Strait, and the Arctic Archipelago-directly connect not with the basin but with the surrounding shelves. Therefore the flushing time for the Canadian Basin halocline is long, and the small amounts of water which leave the basin require little 
shelf water for their replacement. (This effect is not represented in distillery models, where external sources and sinks flush the basin directly.) Nor does the deep water need replacement, because little of it leaves the Canadian Basin in the model solutions. This is corroborated by the long residence times found in the deep Canadian Basin by Östlund et al. (1987) using ${ }^{18} \mathrm{O},{ }^{3} \mathrm{H}$ and ${ }^{14} \mathrm{C}$, and by the paucity of chlorofloromethanes observed by Wallace and Moore (1985) as deep as $1800 \mathrm{~m}$.

Both interpretations show that in steady state, more dense water must be formed in the Eurasian Basin. Taken together, they indicate the importance of the Arctic Archipelago outflow. In the present model, the AA outflow balances mass forcing to the Canadian Basin shelves, and doesn't draw any water from the Canadian Basin halocline. But if the true source of the AA outflow is in the Canadian Basin, and not on the shelves, then both these statements will be reversed. The amount of Canadian Basin dense water formation needed for steady state will increase, as was shown in Section 4.4.2.

Martin and Cavalieri (1989) and Cavalieri and Martin (1994) used satellite and meteorological data to estimate the brine rejection due to freezing in Arctic polynyas, and they calculated the volume of dense water so generated to be just under $1 \mathrm{~Sv}$ (excluding the dense water formed in Bering Sea polynyas south of Bering Strait). ${ }^{2}$ Of this, approximately half was formed on the Canadian Basin shelves, and half on the Eurasian. The present results agree with Cavalieri and Martin in the Canadian Basin, but in the Eurasian Basin, this work finds a greater volume of more saline overflows than did Cavalieri and Martin. Their estimation procedure focuses on production in polynyas, while the present work encompasses

\footnotetext{
${ }^{2}$ This dense water is excluded because it is formed outside of the control area of the present study. Muench et al. (1988) concluded that although Bering Sea dense water does not appear to flow immediately northward to the Bering Strait, it may be flushed through the Strait later in the spring. This would increase the average salinity of the Bering Strait input. In the present work, the Bering Strait salinity is based on the estimates of Rudels (1987) and of Roach et al. (1995), both of which recognize a spring increase in Bering Strait salinity. Thus, the import of Bering Sea dense water may already be accounted for in the present calculations.
} 
all dense water formation. This implies that in steady state, some dense water must be produced on Eurasian Basin shelves somewhere other than in polynyas. That implication strongly supports the growing consensus that the saline Barents Sea inflow is transformed into dense water on its way to the Kara sea, from which it flows into the Eurasian Basin halocline (Steele et al., 1995; Mauritzen, 1996a, b; Schauer et al., 1997).

A complete understanding of shelf-basin exchange comprises three elements:

1. Source: Which waters feed the shelves?

2. Properties: What determines the volume and properties of water leaving the shelves?

3. Distribution: How does water leave the shelf, and where does it go?

This study has demonstrated that proper representation of the shelves' external sources leads to more robust steady solutions, and that shelfbreak upwelling as a source is not important for steady solutions. It has also shown that adding surface mixed layer exchange as a second distribution mechanism (after dense water transport) greatly increases the range of consistent solutions. But many questions remain, especially concerning the properties and distribution of the shelf export, which were left free in the model, subject only to conservation requirements.

There are two different approaches to determining the properties and distribution of water leaving the Arctic shelves. The first is to predict it from first principles. Huthnance (1995) provides a detailed review of just how little is known about the physical processes of cross-shelfbreak exchange. However, primitive equation model studies have yielded important insights about the dynamic limits on the maximum density of water formed in polynyas (Gawarkiewicz and Chapman, 1995; Chapman and Gawarkiewicz, 1997; Spall and Chapman, 1998; Chapman, 1998) and about mechanisms for its distribution (Chapman and Gawarkiewicz, 1995; 
Gawarkiewicz, in prep.), and may soon be able to predict the volume and density distribution of polynya water as a function of initial density and external conditions. Such constraints would be very powerful in determining the consistency of steady models with Arctic observations. This study has chosen a minimalist approach: the only physical constraints are those of steady-state conservation. While ad hoc approximations of physical processes might have been used, the lack of these in the model has made the results very robust. Because the present calculations obey only conservation, any steady-state estimate ought to fall within the ranges calculated here, regardless of the physical constraints assumed.

The second approach is the one used here, viz., inference of shelf water properties and transport from observed tracer distributions. This approach has three components: determination of boundary fluxes, determination of tracer distributions, and the selection of a flow network model to represent possible fluid pathways. These will be discussed in turn.

This study has shown the need for particular attention to the boundary fluxes, as these determine the quantity and nature of shelf-basin fluxes required to maintain steady state. The West Spitzbergen Current is the largest mass flux and also the largest contributor to the uncertainty of the mass budget, and is therefore a good target: have previous estimates been too high? Or is the estimated net outflow of the other passages too low? More important for shelf-basin exchange, though, are the upper ocean fluxes: the Barents inflow, the upper East Greenland Current, and the Arctic Archipelago outflow. Given the scant observational data of these fluxes, the accuracies ascribed to them in this study are almost certainly exaggerated. For example, even if the estimated properties of the Arctic Archipelago outflow are accurate, they surely reflect an unknown contribution of air-sea interaction in the channels of the Archipelago itself, which has not been taken into account. An accurate assessment of which waters are being drained, and at what rate, is crucial to indirect estimates of compensating formation rates. 
The successful introduction of $\delta^{18} \mathrm{O}$ to the box model raises a question: would additional tracers, if added as well, further confirm and refine the conclusions of this study? Or might they contradict the results derived so far, forcing reconsideration of these findings? Two types of tracers present themselves as possibilities: biochemical tracers, and radioisotopes.

Björk (1990) coupled his distillery model (1989) with a simple model of biochemical activity to investigate whether his model could explain the robust nutrient maximum observed at $100 \mathrm{~m}$ depth. Incorporating nitrate, phosphate, and silicate into his model, he not only recaptured the nutrient maximum, but was able to do so with several alternative circulation schemes. He concluded that the nutrient data were too sparse, and biological and physical shelf processes too unknown, for the model to distinguish between the several explanations. It is to be expected that for the time being, attempts to include nutrients in the box model of this study would be similarly inconclusive. Given their large error bars, their inclusion would be easily consistent with the present findings-especially considering the fact that the present box model has many more degrees of freedom than did Björk's.

Radioisotopes have been used frequently in the Arctic to estimate residence times for various regions. If the Arctic basin is ventilated from the shelves, then basin ventilation rates should give some indication of the rate of shelf-basin exchange. For the present, though, there is some debate about residence and ventilation time scales in the upper Arctic. Using a combination of transient and conservative tracers, several authors find residence times for the surface layer and halocline on the order of a decade (Östlund and Hut, 1984; Wallace and Moore, 1985; Schlosser et al., 1994). However, by propagating tritium through Björk's (1989) distillery model, Becker and Björk (1996) find that the time scale of ventilation varies with depth and with model assumptions, spanning scales from decades to centuries. They conclude that the upper Arctic residence times obtained by chemical methods represent averages over an unknown distribution. This suggests that 
transient tracers constraints are unlikely to significantly alter the estimates given by the box model of the present study-all the more so, again, given the strict constraints of their distillery model relative to the present model.

While the current body of tracer data does not appear to be able to constrain the model any better than was done by adding $\delta^{18} \mathrm{O}$, tracer studies do indicate that improvement is needed in the third element of the calculation: the network of boxes and allowed flows which make up the model. Schlosser et al. (1994) examine the residence time of runoff on the Siberian shelves, and find it to be $4 \pm 2$ years. In contrast, the residence time of $0.1 \mathrm{~Sv}$ of runoff in a well-mixed box with the volume of the Arctic shelf seas is approximately 100 years. The discrepancy indicates that runoff must not mix completely into the shelf sea waters, but instead must proceed somewhat coherently to the basin by some path. ${ }^{3}$ The silicate tongue observed north of the Chukchi shelf break by Treshnikov (Bauch et al. 1995, Figure 9) may indicate something of the kind for the Bering Strait inflow. These coherent flows of external water across the shelves are not resolved in the present model.

In general, box models are encumbered by the assumption that their boxes are well-mixed. The present model partly overcomes that limitation in the shelf boxes, by representing dense water of various salinities as well as surface mixed layer water. Nonetheless it is still a box model, as is made clear by the diffusion experiments of Section 4.4.4. As discussed in Section 4.4.1, one feature of the Arctic $\delta^{18} \mathrm{O}$ distribution is the minimum located in the surface layer of the Canadian Basin. When diffusion was set above a threshold value, the loss of $\delta^{18} \mathrm{O}$ from the Canadian Basin surface layer was too great to be explained by data uncertainty, and no consistent solution could be found. In nature, the only source of low $-\delta^{18} \mathrm{O}$ water is runoff, which in the model is mixed into the shelf box, and the average shelf $\delta^{18} \mathrm{O}$ value is advected to the basin. ${ }^{4}$ This is another indication that some amount

\footnotetext{
${ }^{3}$ Though this is true, Bauch (1995) concludes that the runoff probably does not move directly north off the shelf.

${ }^{4}$ One might suppose it is possible to handle this defect as was done for salinity, namely, for
} 
of runoff must make its way more or less coherently from the river mouths to the central basin; otherwise the observed $\delta^{18} \mathrm{O}$ minimum could not be maintained. An alternative explanation is that the prescribed shelf $\delta^{18} \mathrm{O}$ values are wrong, and that the true values are actually lower than in the basin. But that hypothesis was tested in Section 4.3.4, when the shelf $\delta^{18} \mathrm{O}$ was varied. Cases in which the shelves were isotopically lighter than the basins still did not have consistent solutions. Lowering the shelf $\delta^{18} \mathrm{O}$ value created a new problem, viz., not having enough runoff available to dilute the incoming Atlantic and Pacific water down to the lower $\delta^{18} \mathrm{O}$ values.

As was shown in Section 4.4.1, diverting some runoff straight to the basin allows a somewhat wider range of solutions, but the effect is not dramatically large. The final message seems to be that consistent steady-state solutions require surface $\delta^{18} \mathrm{O}$ values to be arranged more or less as they are observed, and preclude large amounts of surface-layer mixing between shelves and basin. But consistent solutions are possible with all runoff flowing to the shelves, or all flowing to the basins, or some flowing to each, in any ratio. Previous models' choice to route runoff and the Bering Strait inflow directly to the basin has been shown here to be a poor choice (Chapter 5). However, the present model's remedy-channeling those flows into well-mixed shelf boxes-is equally simplistic. Reality lies somewhere in between these representations, and the present combination of model and data is not sufficiently powerful to pin down just where it lies.

Getting it right will be crucial for understanding Arctic shelf-basin exchange. Shelf mixing (or the lack thereof) directly affects the amount of nutrients, pollutants, etc. carried from shelf to basin (Becker and Björk, 1996). And smaller-scale flows are important in the basin as well: as mentioned above, the distribution of sources and sinks is the primary factor determining the shelf basin exchange re-

the surface layer exchange to carry the average value of the top $30 \mathrm{~m}$ of the shelf. Unfortunately, shelf profiles of $\delta^{18} \mathrm{O}$ are not available in adequate numbers to determine the average $\delta^{18} \mathrm{O}$ as a function of depth. Computing an approximate shelf surface $\delta^{18} \mathrm{O}$ in proportion to the salinity yields values which are still higher (isotopically heavier) than the basin surface boxes. 
quired for steady state. But the ultimate source of the Arctic Archipelago outflow is completely unknown! Is the Nares Strait outflow drawn from the Eurasian Basin halocline, as represented here, or from the Canadian Basin, or from the western Arctic Archipelago via some boundary current? Is the western Archipelago filled primarily from the Canadian Basin, or from the Chukchi Sea and Canadian rivers via the Beaufort Shelf? Likewise, better knowledge of the entry path of West Spitzbergen Current waters would help pin down the density distribution of shelf water required for steady-state (Section 4.4.3). Observations clarifying these issues would go a long way toward correct definition of pathways in the model, and toward pinning down the shelf-basin exchange required for steady state.

Efforts toward this goal are proceeding. Thomas et al. (1996) show the viability of combining predicted winds with ice drift observations. Such techniques could provide some information on the location and frequency of cross-shelfbreak transport in the surface mixed layer, which could be used to refine the representation of that transport in the model. Additionally, efforts are being made to identify new tracers which mark shelf water (Falkner et al., 1994; Rutgers van der Loeff et al., 1995; Edmonds et al., 1998; Smith et al., 1998), but like the nutrients and transient tracers already in use, their full potential will not be realized until their distribution and mechanics can be more accurately estimated.

Progress will occur most rapidly, of course, if new dynamical constraints, improved boundary flux estimates, a stronger tracer database, and more judicious choices of allowed pathways are all included in one model, allowing their various strengths to compensate for their individual defects.

As the database improves, it will be possible to constrain ever-more complicated models. Finer space and time scales would allow resolution of important processes which exist on scales smaller than the basin and multi-year scales. One possibility would be to resolve the various shelf seas. They differ in their salinities, topographies, and atmospheric conditions, and their dense water output may 
therefore vary as well (Aagaard et al., 1981; Martin and Cavalieri, 1989; Cavalieri and Martin, 1994; Thomas et al., 1996; Schauer et al., 1997). Likewise, the Arctic basins contain potentially important small-scale structure, such as bottomtrapped boundary currents at the shelf break (Aagaard, 1989), with which dense shelf plumes might mix as they descend (Rudels et al. 1994; Jones et al. 1995). It must also be kept in mind that even if the present solutions are accurate assessments of the average Arctic state, that state is probably not ever the actual state of the Arctic at a given moment. A periodic version of the model, while not strictly steady, could be used to determine whether there is an average Arctic seasonal cycle consistent with the current data. Inflow properties, such as those of the Bering Strait, are known to vary seasonally, and dense water production and ice melt are surely seasonal. Correlations between the seasonal variations of water properties and circulation may well be significant. On the other hand, if shelf residence times are indeed many years long, the shelves may integrate over many seasonal cycles, reducing the importance of resolving the annual timescale. Ultimately a variety of scales will have to be resolved. For example, runoff may linger on the shelves for years before entering the basins, while dense water may fall over the shelfbreak immediately upon formation.

\subsection{Steady Models and Arctic Change}

The failure of the data so far to contradict the steady hypothesis in no way guarantees the stationarity of the Arctic circulation. Just as likely is the possibility that change is occurring, but slowly enough to fit inside the ample error bars of our estimates. However, the steady problem is an important baseline from which to discuss questions of change. This work has generated a picture of the steady Arctic wherein processes of water formation balance processes of water removal. Changes in the thermohaline structure of the Arctic will necessarily follow any alteration of 
that balance.

In general, the steady state box model estimates how much dense water must be formed in each basin in order to (a) maintain conservation on the shelf, and (b) replace water which exits the basin. Of course, the physical mechanisms which determine actual dense water may not meet these needs, in which case steady state will not be maintained. Take, as an example, the response to increased runoff. It was shown in Section 4.5.1 that to a point, changes in runoff don't directly affect the deep water formation requirements for steady state. However, an increase in runoff might increase the fraction of Polar Water which exits through the Arctic Archipelago (Steele et al., 1996), which could affect the location from which Polar Water was removed from the Arctic. As has been shown, the steady state demand for shelf water depends on the volume and location of water removed from the system. If runoff were to increase, both the pattern of drainage from the Arctic and the production of dense water on the shelves would probably be affected. There is no reason to suppose that the changes in dense water formation would be exactly those needed to balance the new outflow demands and maintain the current thermohaline structure.

Recognizing that in steady state, the shelves are fed from external sources and not from the basin surface mixed layer untangles a potential feedback mechanism. If the main source for the shelves were the basins, then a change in basin profiles could affect the shelf water properties, which would affect dense water formation, further affecting the basin profiles. The present results indicate that shelf processes are probably independent of the basin properties. However, the shelf processes could very much be affected by changes in the shelves' external sources. Such changes would then propagate into the basins. 


\subsection{Closing}

Steady state in the Arctic is a balance between inflows, outflows, and internal formation processes-and the shelves play a role in all three. Understanding the processes active on the shelves, and the means of cross-shelfbreak transport, is paramount to comprehending how steady state in the Arctic is, or is not, maintained. In the past, the role of shelf-basin exchange in the Arctic has been evaluated by determining the amount consistent with observed temperature and salinity profiles. The present work has shown that a wide range of cross-shelfbreak transports meet this criterion, and therefore, that a given circulation scheme's ability to produce the correct temperature and salinity profiles is not enough to prove that scheme correct. Successful estimates must exploit further information, such as additional tracers, or well-founded dynamic constraints. Furthermore, shelf sources and sinks must be accurately represented, or estimates of shelf-basin exchange will be unnaturally constrained.

This study has demonstrated how much can be inferred by combining data and estimates from all available sources, "not swearing by any one of them but exploiting their quarrelsome conference." ${ }^{5}$ There is great potential in the volume of incoming data, but much of that potential will be wasted if the information is not continually and quantitatively synthesized, using the data uncertainties, to form a constantly improving, comprehensive picture of the Arctic. To the extent that the data are consistent with the steady model, our understanding of the average Arctic circulation will become ever more accurate. And if, as has not yet happened, those data should one day significantly conflict with the steady model, the contradictions will provide valuable clues to the mechanisms of Arctic change.

\footnotetext{
${ }^{5}$ Umberto Eco, The Island of the Day Before, New York, Harcourt Brace \& Co., 1995, 515 pp, Ch. 23: "Father Caspar replied that while all were erroneous when taken one by one, if taken together the various results could achieve a balance and compensate for the individual defects: 'And this est mathematica!'" (tr. William Weaver)
} 


\section{Appendix A}

\section{Solution Uncertainty in}

\section{Non-Negative Least Squares}

Thomson (1982) ${ }^{1}$ worked out the error of one solution element in an NNLS problem with two unknowns. The result was complicated enough to discourage an attempt with over 80 unknowns. However, it is feasible under certain conditions to examine the error of a linear approximation to the NNLS problem. Sadly, the systems considered in this study did not meet those conditions, and so the approach was of no use to the present project. Nonetheless the approach may find later application, and so is presented here.

Given a matrix $\mathbf{E}$, the non-negative least squares (NNLS) algorithm can be considered a non-linear function of a data vector $\mathbf{f}$, which returns the solution $\hat{\mathbf{x}}$ to the NNLS problem $\mathbf{E x}+\mathbf{n}=\mathbf{f}, \mathbf{x} \geq \mathbf{0},\|\mathbf{n}\|$ to be minimized. If $\mathbf{f}$ is known only to within a specified uncertainty, it follows that $\hat{\mathbf{x}}$ can only be estimated to within some uncertainty, whose size we would like to determine. This is equivalent to asking, what is the change $\mathbf{d x}$ in the solution given a change $\mathbf{d f}$ in the data? That question is addressed here.

\footnotetext{
${ }^{1}$ See also Davis (1978).
} 
The solution $\hat{\mathbf{x}}$ to the NNLS problem will in general have elements $\hat{\mathbf{x}}(z)$ which equal zero, and elements $\hat{\mathbf{x}}(p)$ which are positive. Here, $p$ and $z$ represent sets of element indices. The solution is accompanied by a dual vector $\hat{\mathbf{w}}=\mathbf{E}^{\mathrm{T}}(\mathbf{f}-\mathbf{E} \hat{\mathbf{x}})$, which is the negative gradient vector of the cost function $\frac{1}{2}\|\mathrm{Ex}-\mathbf{f}\|^{2}$ at $\mathbf{x}=\hat{\mathbf{x}}$. Its elements, corresponding to those in $\hat{\mathbf{x}}$, are negative $(\hat{\mathbf{w}}(z))$ or zero $(\hat{\mathbf{w}}(p))$. Most important for the present purpose is the fact that $\hat{\mathbf{x}}(p)$, the non-zero subset of the NNLS solution, is also the solution of the unconstrained least squares problem $\mathbf{E}_{p} \mathbf{x}(p)+\mathbf{n}=\mathbf{f}$, where $\mathbf{E}_{p}=\mathbf{E}(:, p)$, i.e., $\mathbf{E}_{p}$ is formed from the columns of $\mathbf{E}$ whose indices are listed in $p$. Lawson and Hanson (1974) give details.

At $\hat{\mathbf{x}}$, then, the complicated NNLS operator is represented by the much simpler rules $\hat{\mathbf{x}}(p)=\mathbf{E}_{p}^{+} \mathbf{f}, \hat{\mathbf{x}}(z)=\mathbf{0}$. It seems reasonable to postulate that this is true not only at $\hat{\mathbf{x}}$, but also in a neighborhood of $\hat{\mathbf{x}}$, and that a perturbation $\mathbf{d f}$ to the data will result in a change in the NNLS solution $\mathbf{d x}=\mathrm{E}_{p}^{+} \mathbf{d f}$. While this seems plausible, it is by no means evident: perhaps any perturbation $\mathbf{d f}$ is enough for the NNLS solution to change character, that is, for an element of $\hat{\mathbf{x}}$ which was zero to become positive, or vice versa. In this case, different columns of the original $\mathbf{E}$ would form the appropriate $\mathbf{E}_{p}$, and error analysis based on the first $\mathbf{E}_{p}$ would be irrelevant.

It turns out, however, that there is a finite and determinable neighborhood of $\hat{\mathbf{x}}$ for which error analysis based on $\mathbf{E}_{p}^{+}$is an accurate analysis of the NNLS operator. This is shown by determining the conditions under which $\tilde{\mathbf{x}}$, the solution to $\mathbf{E}_{p} \mathbf{x}+\mathbf{n}=(\mathbf{f}+\mathbf{d f})$, is also the solution to the NNLS problem $\mathbf{E x}+\mathbf{n}=(\mathbf{f}+\mathbf{d f})$, $\mathbf{x} \geq \mathbf{0}$. To do so, we determine the circumstances under which $\tilde{\mathbf{x}}$ satisfies the Kuhn-Tucker characterization of the NNLS solution, for which see Lawson and Hanson (1974).

Define $\tilde{\mathbf{x}}$ by $\tilde{\mathbf{x}}(p)=\mathbf{E}_{p}^{+}(\mathbf{f}+\mathbf{d f}), \tilde{\mathbf{x}}(z)=\mathbf{0}$, and define $\tilde{\mathbf{w}}=\mathbf{E}^{\mathrm{T}}(\mathbf{f}+\mathbf{d f}-\mathbf{E} \tilde{\mathbf{x}})$. Note that 
1. $\tilde{\mathbf{x}}(z)=\mathbf{0}$, by definition.

2. $\tilde{\mathbf{w}}(p)=0$. Proof:

$$
\begin{aligned}
\tilde{\mathbf{w}}(p) & =\mathbf{E}^{\mathrm{T}}(\mathbf{f}+\mathbf{d f}-\mathbf{E} \tilde{\mathbf{x}}) \\
& =\mathbf{E}^{\mathrm{T}}\left(\mathbf{f}+\mathbf{d f}-\mathrm{E}_{p} \tilde{\mathbf{x}}(p)\right) \text { since } \tilde{\mathbf{x}}(z)=\mathbf{0} \\
& =\mathbf{E}^{\mathrm{T}}\left(\mathbf{f}+\mathbf{d f}-\mathrm{E}_{p} \mathrm{E}_{p}^{+}(\mathbf{f}+\mathbf{d f})\right) \\
& =\left(\mathrm{E}^{\mathrm{T}}{ }_{p}-\mathbf{E}^{\mathrm{T}}{ }_{p} \mathrm{E}_{p} \mathrm{E}_{p}^{+}\right)(\mathbf{f}+\mathbf{d f}) \\
& =\mathbf{0} .
\end{aligned}
$$

This means that as long as $\tilde{\mathbf{x}}(p)>\mathbf{0}$ and $\tilde{\mathbf{w}}(z) \leq \mathbf{0}, \tilde{\mathbf{x}}$ is the NNLS solution to $\mathbf{E x}+\mathbf{n}=\mathbf{f}+\mathbf{d f}$ by the Kuhn-Tucker theorem.

The usefulness of this idea for determining the uncertainty of the NNLS solution can be evaluated as follows. Given $\mathbf{E x}+\mathbf{n}=\mathbf{f}$, scaled so that $\left\langle\mathbf{n} \mathbf{n}^{\mathbf{T}}\right\rangle=\mathbf{I},\langle\mathbf{n}\rangle=\mathbf{0}$ (where $\langle\cdot\rangle$ is the expected value),

1. Use the NNLS algorithm to determine $\hat{\mathbf{x}}, \hat{\mathbf{w}}, \mathrm{E}_{p}$.

2. Make the provisional assumption that the NNLS operator can be expressed as $\mathbf{E}_{p}^{+}$. Evaluate the uncertainty of $\hat{\mathbf{x}}(p)$ and $\hat{\mathbf{w}}(z)$ as follows:

$$
\begin{aligned}
& \hat{\mathbf{w}}=\mathbf{E}^{\mathrm{T}}(\mathbf{f}-\mathbf{E} \hat{\mathbf{x}}) \\
& \Rightarrow \quad \mathbf{I}(:, z) \hat{\mathbf{w}}(z)=\mathbf{E}^{\mathrm{T}}\left(\mathbf{f}-\mathbf{E}_{p} \hat{\mathbf{x}}(p)\right) \text { since } \hat{\mathbf{x}}(z), \hat{\mathbf{w}}(p)=\mathbf{0} \\
& \Rightarrow \quad \mathbf{E}^{\mathrm{T}} \mathbf{E}_{p} \hat{\mathbf{x}}(p)+\mathbf{I}(:, z) \hat{\mathbf{w}}(z)=\mathbf{E}^{\mathrm{T}} \mathbf{f} \\
& \Rightarrow\left[\begin{array}{ll}
\mathbf{E}^{\mathrm{T}} \mathbf{E}_{p} & \mathrm{I}(: ; z)
\end{array}\right]\left(\begin{array}{c}
\hat{\mathbf{x}}(p) \\
\hat{\mathbf{w}}(z)
\end{array}\right)=\mathbf{E}^{\mathrm{T}} \mathbf{f}
\end{aligned}
$$

Define $\mathbf{A}$ and $\hat{\mathbf{y}}$ respectively as the matrix and the vector which appear on the right hand side. Then

$$
A \hat{\mathbf{y}}=E^{T} \mathbf{f}
$$


from which it can be shown that

$$
\mathbf{P}_{\hat{\mathbf{y}} \hat{\mathbf{y}}} \equiv\left\langle(\hat{\mathbf{y}}-\langle\hat{\mathbf{y}}\rangle)(\hat{\mathbf{y}}-\langle\hat{\mathbf{y}}\rangle)^{\mathrm{T}}\right\rangle=\mathbf{A}^{+} \mathbf{E}^{\mathrm{T}} \mathbf{E} \mathbf{A}^{+\mathrm{T}}
$$

The diagonal of $\mathrm{P}_{\hat{\mathbf{y}} \hat{\mathbf{y}}}$ contains the variances of $\hat{\mathbf{x}}(p)$ and $\hat{\mathbf{w}}(z)$.

3. If the uncertainty of $\hat{\mathbf{y}}$ is small enough that the sign of each element is well determined, then $\hat{\mathbf{x}}(p)$ will remain positive for any expected perturbation of $\mathbf{f}$, and $\hat{\mathbf{w}}(z)$ will remain negative. Therefore, $\mathbf{E}_{p}^{+}$is an exact representation of the NNLS operator for the expected range of the data, justifying the assumption made in step 2. A full linear analysis of the operator is then possible. On the other hand, if any element of $\hat{\mathbf{y}}$ is not significantly different from zero, then there exist plausible perturbations to $\mathbf{f}$ which are large enough to change the character of the NNLS solution, by including elements which were zero in $\hat{\mathbf{x}}$, by zeroing elements which were positive in $\hat{\mathbf{x}}$, or both. In that case, the assumption made in step 2 that $\mathrm{E}_{p}^{+}$represents the NNLS operator does not hold for all likely perturbations of the data, so the solution error computed based on that assumption is invalid.

In sum, if the NNLS algorithm defined by a matrix $\mathbf{E}$ is viewed as an operator on the data vector $\mathbf{f}$, that operator may be described as "piecewise linear", in that it can be represented by a constant matrix for a certain range of $\mathbf{f}$. Outside that range, the operator takes the form of different matrices, each of which is constant over its own associated range of $\mathbf{f}$. For a given solution, it is possible using (A.1) and (A.2) to determine in some sense the range of $\mathbf{f}$ for which $\mathbf{E}_{p}^{+}$accurately represents the NNLS algorithm, and for which the error analysis of $\mathbf{E}_{p}^{+}$is the error analysis of the NNLS operator. 


\section{Appendix B}

\section{Constraining Ice Formation with the Estimates of Thomas et al.}

Rates of ice melt and formation were extracted from the inverse study of Thomas et al. (1996). This process was somewhat haphazard as the seven cells of their model did not correspond closely with the four surface cells of the box model. Furthermore, Thomas et al. did not include the Barents Sea in their study. Nonetheless I went ahead and made the identifications listed in Table B.1. Note that as elsewhere in this report, ice volumes have been multiplied by 0.9 to convert to equivalent liquid volumes.

The error estimates listed in Table B.1 were generated as follows. To the error

Table B.1: Net ice melt (melt-freeze) for each surface box, based on the results of Thomas et al. (1996). Ice volumes have been multiplied by 0.9 to give equivalent liquid volumes.

\begin{tabular}{llr}
\hline \multicolumn{1}{c}{ Thomas et al.'s cells } & \multicolumn{1}{c}{ My box } & melt-freeze (Sv) \\
\hline Canada Basin, Central Arctic, 1/2 N. Pole & Canadian Basin & $0.00 \pm 0.03$ \\
Beaufort, Chukchi & Canadian Basin Shelves & $-0.01 \pm 0.01$ \\
Nansen Basin, 1/2 N. Pole & Eurasian Basin & $-0.02 \pm 0.01$ \\
Laptev & Eurasian Basin Shelves & $-0.01 \pm 0.02$ \\
\hline
\end{tabular}


values listed under " $\mathrm{sd}_{\tau}$ " in Table 2 of Thomas et al., I added $0.375 \mathrm{~m}$. This represented $1 / 2$ the $0.75 \mathrm{~m}$ average difference between Thomas et al.'s estimates and submarine sonar measurements, and was used to reflect systematic errors in the estimation (D Thomas, personal communication, 1996). I then took the standard deviation of the seven values thus augmented to obtain the standard error for net ice growth, and multiplied by the cell area to determine net ice formation.

As Table B.1 shows, the ice formation rates become very uncertain when mapped to the coarse resolution of the box model. Additional equations were appended to the model, constraining it to match these ice formation rates, producing a system of 99 constraints on 95 unknowns, with a numerical rank of 88 . (The additional constraints did not change the rank of the system, but did flatten the distribution of the smallest singular values.) As might have been predicted given the high uncertainty of the new constraints, consistent solutions were easily found. 


\section{References}

Aagaard K (1981) On the deep circulation in the Arctic Ocean. Deep-Sea Research, 28, 251-268.

Aagaard K (1989) A synthesis of the Arctic Ocean circulation. Rapp. P.-v. Réun. Cons. int. Explor. Mer, 188, 11-22.

Aagaard K and E C Carmack (1989) The role of sea ice and other fresh water in the Arctic circulation. Journal of Geophysical Research, 94, 14,485-14,498.

Aagaard K and E C Carmack (1994) The Arctic Ocean and climate: a perspective. In The Polar Oceans and Their Role in Shaping the Global Environment, O M Johannessen, R D Muench and J E Overland, eds., Geophysical Monograph Series Number 85, American Geophysical Union, Washington, D. C., 525 pp.

Aagaard K and L K Coachman (1975) Toward an ice-free Arctic Ocean. EOS, 56, 484-486.

Aagaard K, L K Coachman and E Carmack (1981) On the halocline of the Arctic Ocean. Deep-Sea Research I, 28, 529-545.

Aagaard K and P Greisman (1975) Toward new mass and heat budgets for the Arctic Ocean. Journal of Geophysical Research, 80, 3,821-3,827.

Aagaard K and A T Roach (1990) Arctic ocean-shelf exchange: measurement in Barrow Canyon. Journal of Geophysical Research, 95, 18,163-18,175.

Aagaard K, J H Swift and E C Carmack (1985) Thermohaline circulation in the Arctic Mediterranean Seas. Journal of Geophysical Research, 90, 4,8334,846 .

Addison V G (1987) The Physical Oceanography of the Northern Baffin Bay-Nares Strait Region. MA thesis, Naval Post-Graduate School, Monterey, CA.

Bauch D (1995) The Distribution of $\delta^{18} O$ in the Arctic Ocean: Implications for the Freshwater Balance of the Halocline and the Sources of Deep and Bottom 
Waters. Reports on Polar Research 159, Alfred Wegner Institute for Polar and Marine Research, Bremerhaven, Germany.

Bauch D, P Schlosser and R G Fairbanks (1995) Freshwater balance and the sources of deep and bottom waters in the Arctic Ocean inferred from the distribution of $\mathrm{H}_{2}{ }^{18} \mathrm{O}$. Progress in Oceanography, 35, 53-80.

Becker P and G Björk (1996) Residence times in the upper Arctic Ocean. Journal of Geophysical Research, 101, 28,377-28,396.

Björk G (1989) A one-dimensional time-dependent model for the vertical stratification of the upper Arctic Ocean. Journal of Physical Oceanography, 19, $52-67$.

Björk G (1990) The vertical distribution of nutrients and oxygen 18 in the upper Arctic Ocean. Journal of Geophysical Research, 95, 16,025-16,036.

Blindheim J (1989) Cascading of Barents Sea bottom water into the Norwegian Sea. Rapp. P.-v. Réun. Cons. int. Explor. Mer, 188, 49-58.

Bönisch G and P Schlosser (1995) Deep water formation and exchange rates in the Greenland/Norwegian Seas and Eurasian Basin of the Arctic Ocean derived from tracer balances. Progress in Oceanography, 35, 29-52.

Bunker A F and L V Worthington (1976) Energy exchange charts of the North Atlantic Ocean. Bulletin of the American Meteorological Society, 57, 670678.

Bush J W M and A W Woods (1998) Eddy generation in the Arctic Ocean through freezing of linear fractures in the ice cover. Nature, submitted.

Carmack, E C (1986) Circulation and mixing in ice-covered waters. In The Geophysics of Sea Ice, N Untersteiner, ed., NATO ASI Series B, 146, Plenum Press, New York, 1,196 pp.

Carmack, E C, R W Macdonald, R G Perkin, F A McLaughlin and R J Pearson (1995) Evidence for warming of Atlantic water in the southern Canadian Basin of the Arctic Ocean: results from the Larsen-93 expedition. Geophysical Research Letters, 22, 1,061-1,064.

Cavalieri D J and S Martin (1994) The contribution of Alaskan, Siberian, and Canadian coastal polynyas to the cold halocline layer of the Arctic Ocean. Journal of Geophysical Research, 99, 18,343-18,362.

Chapman D C (1998) Dense water formation beneath a time-dependent coastal polynya. Submitted to Journal of Physical Oceanography. 
Chapman D C and G Gawarkiewicz (1995) Offshore transport of dense shelf water in the presence of a submarine canyon. Journal of Geophysical Research, 100, $13,373-13,387$.

Chapman D C and G Gawarkiewicz (1997) Shallow convection and buoyancy equilibration in an idealized coastal polynya. Journal of Physical Oceanography, $27,555-566$.

Coachman L K and K Aagaard (1974) Physical oceanography of Arctic and subarctic seas. In Marine Geology and Oceanography of the Arctic Seas, Y Herman, ed., Springer-Verlag, New York, 888 pp.

Craig H (1961) Standard for reporting concentration of deuterium and oxygen-18 in natural waters. Science, 133, 1,833-1,834.

D'Asaro E A (1988a) Observations of small eddies in the Beaufort Sea. Journal of Geophysical Research, 93, 6,669-6,684.

D'Asaro E A (1988b) Generation of sub-mesoscale vortices: a new mechanism. Journal of Geophysical Research, 93, 6,685-6,693.

Davis W W (1978) Bayesian analysis of the linear model subject to linear inequality constraints. Journal of the American Statistical Association, 73, $573-579$.

Edmonds, H N, J N Smith, H D Livingston, L R Kilius and J M Edmond (1998) ${ }^{129} \mathrm{I}$ in archived seawater samples. Deep-Sea Research $I$, in press.

Falkner K K, R W Macdonald, E C Carmack and T Weingartner (1994) The potential of barium as a tracer of Arctic water masses. In The Polar Oceans and Their Role in Shaping the Global Environment, O M Johannessen, R D Muench and J E Overland, eds., Geophysical Monograph Series Number 85, American Geophysical Union, Washington, D. C., 525 pp.

Fissel D B, D D Lemon, H Melling and R A Lake (1988) Non-tidal fiows in the Northwest Passage. Can. Tech. Rep. Hydrog. Ocean Sci. 98, Inst. of Ocean Sci., Sidney, B. C., Canada.

Fofonoff N (1977) Computation of potential temperature of sea water for an arbitrary reference pressure. Deep-Sea Research, 24, 489-491.

Foldvik A, K Aagaard and T Tørresen (1988) On the velocity field of the East Greenland Current. Deep-Sea Research I, 35, 1,335-1,354. 
Fu L L (1981) The general circulation and meridional heat transport of the subtropical South Atlantic determined by inverse methods. Journal of Physical Oceanography, 11, 1,171-1,193.

Gawarkiewicz G (in preparation) Effects of ambient stratification and shelfbreak topography on offshore transport of dense shelf water.

Gawarkiewicz G and D C Chapman (1995) A numerical study of dense water formation and transport on a shallow, sloping continental shelf. Journal of Geophysical Research, 100, 4,489-4,507.

Gawarkiewicz G, T Weingartner and D C Chapman (1998) Sea ice processes and water mass modification and transport over Arctic shelves. In The Sea: The Global Coastal Ocean. Processes and Methods, $\mathrm{K}$ H Brink and A R Robinson, eds., John Wiley and Sons, 10, 171-190.

Gore-Chernomyrdin Commission Environmental Working Group (1997) Joint U.S.-Russian Atlas of the Arctic Ocean, Oceanography Atlas for the Winter Period, CD-ROM. National Snow and Ice Data Center, Campus Box 449, University of Colorado, Boulder, CO 80309-0449, <http://ns.noaa.gov/ atlas/ $>$.

Grace A (1990) Optimization Toolbox for use with MATLAB. The MathWorks, Inc., Natick, MA, 56 pp.

Grebmeier, J M et al., eds. (1998) Arctic System Science Ocean-Atmosphere-Ice Interactions Western Arctic Shelf-Basin Interactions Science Plan, ARCSS/ OAII Report Number 7, Old Dominion University, Norfolk, VA, 65 pp.

Häkkinen S (1993) An Arctic source of the Great Salinity Anomaly: a simulation of the Arctic ice-ocean system for 1955-1975. Journal of Geophysical Research, 98, 16,397-16,410.

Hanzlick D J (1983) The West Spitzbergen Current: Transport, Forcing, and Variability. PhD thesis, University of Washington School of Oceanography, Seattle, WA, $127 \mathrm{pp}$.

Hibler W D (1979) A dynamic thermodynamic sea ice model. Journal of Physical Oceanography, $\mathbf{9}, 815-846$.

Huthnance J M (1995) Circulation, exchange and water masses at the ocean margin: the role of physical processes at the shelf edge. Progress in Oceanography, $35,353-431$. 
Jones E P, B Rudels and L G Anderson (1995) Deep waters of the Arctic Ocean: origins and circulation. Deep-Sea Research, 42, 737-760.

Killworth P D and J M Smith (1984) A one-and-a-half dimensional model for the Arctic halocline. Deep-Sea Research, 31, 271-293.

Lawson C L and R J Hanson (1974) Solving Least Squares Problems. PrenticeHall, Englewood Cliffs, NJ, 340 pp.

LeGrand P and C Wunsch (1995) Constraints from paleotracer data on the North Atlantic circulation during the last glacial maximum. Paleocoeanography, 10, 1011-1045.

Lewis E L (1980) The Practical Salinity Scale 1978 and its antecedents. IEEE Journal of Oceanic Engineering, OE-5, 3-8.

Livingston H D (1995) Arctic radioactivity and transport processes: An introduction. Deep-Sea Research II, 42, 1,337-1,339.

Martin S and D J Cavalieri (1989) Contributions of the Siberian Shelf Polynyas to the Arctic Ocean Intermediate and Deep Water. Journal of Geophysical Research, 94, 12,725-12,738.

Mauritzen C (1996a) Production of dense overflow waters feeding the North Atlantic across the Greenland-Scotland Ridge. Part 1: evidence for a revised circulation scheme. Deep-Sea Research I, 43, 769-806.

Mauritzen C (1996b) Production of dense overflow waters feeding the North Atlantic across the Greenland-Scotland Ridge. Part 2: an inverse model. DeepSea Research I, 43, 807-835.

Maykut G (1982) Large-scale heat exchange and ice production in the central Arctic. Journal of Geophysical Research, 87, 7,971-7,984.

Maykut G (1985) An Introduction to Ice in the Polar Oceans. APL-UW 8510, Department of Atmospheric Sciences/Geophysics Program, University of Washington, Seattle, $107 \mathrm{pp}$.

Maykut G and M McPhee (1995) Solar heating of the Arctic mixed layer. Journal of Geophysical Research, 100, 24,691-24,703.

McLaughlin F A, E C Carmack, R W Macdonald and J K B Bishop (1996) Physical and geochemical properties across the Atlantic/Pacific water mass front in the southern Canadian Basin. Journal of Geophysical Research, 101, $1,183-1,197$. 
Melling $\mathrm{H}$ (1993) The formation of a haline shelf front in wintertime in an icecovered arctic sea. Continental Shelf Research, 13, 1,123-1,147.

Melling H and E L Lewis (1982) Shelf drainage flows in the Beaufort Sea and their effect on the Arctic Ocean pycnocline. Deep-Sea Research, 29, 967-985.

Muench R D (1971) The physical oceanography of the northern Baffin Bay region. Baffin Bay-N. Water Proj., Sci. Rep. 1, Arctic Inst. of N. Amer., Montreal, Que.

Muench R D, J D Schumacher and S A Salo (1988) Winter currents and hydrographic conditions on the northern central Bering Sea shelf. Journal of Geophysical Research, 93, 516-526.

NODC (1994) World Ocean Atlas 1994, CD-ROM. National Oceanographic Data Center, User Services Group, NOAA/NESDIS OC1, SSMC3, 4th floor, 1315 East-West Highway, Silver Spring, MD 20910-3282, <http://www.nodc. noaa.gov/index.html >.

Östlund H G and G Hut (1984) Arctic Ocean water mass balance from isotope data. Journal of Geophysical Research, 89, 6,373-6,381.

Östlund H G, G Possnert, and J H Swift (1987) Ventilation rate of the deep Arctic Ocean from carbon 14 data. Journal of Geophysical Research, 92, $3,769-3,777$.

Paquette R G, R H Bourke, J F Newton and W F Perdue (1985) The East Greenland Polar Front in autumn. Journal of Geophysical Research 90, 4,8664,882 .

Priestley M B (1981) Spectral Analysis and Time Series. Academic Press, London, $890 \mathrm{pp}$.

Quadfasel D, B Rudels and K Kurz (1988) Outflow of dense water from a Svalbard fjord into the Fram Strait. Deep-Sea Research, 35, 1,143-1,150.

Roach A T, K Aagaard, C H Pease, S A Salo, T Weingartner, V Pavlov and M Kulakov (1995) Direct measurements of transport and water properties through the Bering Strait. Journal of Geophysical Research, 100, 18,44318,457 .

Rudels B (1986) The outflow of Polar Water through the Arctic Archipelago and the oceanographic conditions in Baffin Bay. Polar Research, 4.

Rudels B (1987) On the mass balance of the Polar Ocean, with special emphasis on the Fram Strait. Norsk Polarinstitutt Skrifter 188. 
Rudels B, E P Jones, L G Anderson and G Kattner (1994) On the intermediate depth waters of the Arctic Ocean. In The Polar Oceans and Their Role in Shaping the Global Environment, O M Johannessen, R D Muench and J E Overland, eds., Geophysical Monograph Series Number 85, American Geophysical Union, Washington, D. C., 525 pp.

Rudels B, L G Anderson and E P Jones (1996) Formation and evolution of the surface mixed layer and halocline of the Arctic Ocean. Journal of Geophysical Research, 101, 8,807-8,821.

Rutgers Van Der Loeff M M, R M Key, J Scholten, D Bauch and A Michel (1995) ${ }^{228} \mathrm{Ra}$ as a tracer for shelf water in the Arctic Ocean. Deep-Sea Research II, $42,1,533-1,553$.

Schauer U, R D Muench, B Rudels and L Timokhov (1997) Impact of eastern Arctic shelf waters on the Nansen Basin intermediate layers. Journal of Geophysical Research, 102, 3,371-3,382.

Schlosser P, D Bauch, R Fairbanks and G Bönisch (1994) Arctic river-runoff: mean residence time on the shelves and in the halocline. Deep-Sea Research $I, 41,1,053-1,068$.

Schlosser P, J Swift, D Lewis and S L Pfirman (1995) The role of the largescale Arctic Ocean circulation in the transport of contaminants. Deep-Sea Research II, 42, 1,341-1,367.

SCOR Working Group 58 (1979) The Arctic Heat Budget. Geophysical Institute Report No. 52, University of Bergen, Norway, 98 pp.

Smith J N, K M Ellis and L R Kilius (1998) ${ }^{129} \mathrm{I}$ and ${ }^{137} \mathrm{Cs}$ tracer measurements in the Arctic Ocean. Deep-Sea Research I, in press.

Smith S D, R D Muench and C Pease (1990) Polynyas and leads: an overview of physical processes and environment. Journal of Geophysical Research, 95, $9,461-9,479$.

Spall M A and D C Chapman (1998) On the efficiency of baroclinic eddy transport across narrow fronts. Journal of Physical Oceanography, in press.

Steele M, J H Morison and T B Curtin (1995) Halocline water formation in the Barents Sea. Journal of Geophysical Research, 100, 881-894.

Steele M, D Thomas, D Rothrock and S Martin (1996) A simple model study of the Arctic Ocean freshwater balance, 1979-1985. Journal of Geophysical Research, 101, 20,833-20,848. 
Strang G (1993) Introduction to Linear Algebra. Wellesley-Cambridge Press, Wellesley, MA, 472 pp.

Thomas D, S Martin, D Rothrock and M Steele (1996) Assimilating satellite concentration data into an Arctic sea ice mass balance model, 1979-1985. Journal of Geophysical Research, 101, 20,849-20,868.

Thomson, M (1982) Some results on the statistical properties of an inequality constrained least squares estimator in a linear model with two regressors. Journal of Econometrics, 19, 215-231.

Triperman E and A Hecht (1987) A note on the circulation in the eastern Levantine basin by inverse methods. Journal of Physical Oceanography, 18, $506-518$.

Vinje $T$ and $\varnothing$ Finnekåsa (1986) The ice transport through the Fram Strait. Norsk Polarinstitutt Skrifter 186.

Vinje T, N Nordlund, S Østerhus, R Korsnes, Å Kvambekk and E Nøst (1997) Monitoring ice thickness in Fram Strait. Submitted to Journal of Geophysical Research.

Wallace, D W R and R M Moore (1985) Vertical profiles of $\mathrm{CCl}_{3} \mathrm{~F}$ (F-11) and $\mathrm{CCl}_{2} \mathrm{~F}_{2}$ (F-12) in the central Arctic Ocean basin. Journal of Geophysical Research, 90, 1,155-1,166.

Wunsch, C (1996) The Ocean Circulation Inverse Problem. Cambridge University Press, New York, 442 pp. 